\title{
Studies Toward the Synthesis of Amaryllidaceae Alkaloids from Morita-Baylis-Hillman Adducts. A Straightforward Synthesis of Functionalized Dihydroisoquinolin-5(6H)-one Core
}

\author{
Elizandra C.S. Lopes and Fernando Coelho*
}

Instituto de Química, Universidade Estadual de Campinas, CP 6154, 13083-970 Campinas-SP, Brazil

\begin{abstract}
Descrevemos neste trabalho, os resultados de um estudo que teve como objetivo a síntese de esqueletos de carbono altamente funcionalizados de alcalóides de plantas da família das Amaryllidaceae. A partir de adutos de Morita-Baylis-Hillman, descrevemos a síntese total do núcleo diidroisoquinolin-5(6H)-ona funcionalizado, que é a parte inferior da estrutura de vários alcalóides dessa classe. Essa substância pode ser um intermediário útil e valioso para a síntese total dos alcalóides isolados de plantas da família Amaryllidaceae.
\end{abstract}

We disclose herein our results concerning a study aiming at the synthesis of the highly substituted carbon skeleton of alkaloids isolated from plants of the Amaryllidaceae family. The total synthesis of the functionalized dihydroisoquinolin-5(6H)-one core, which is the bottom part of the structure of alkaloids isolated from this botanic family, is described, using MoritaBaylis-Hillman adducts as substrate. This compound should be a useful and valuable intermediate for the total synthesis of alkaloids isolated from Amaryllidaceae.

Keywords: Morita-Baylis-Hillman adducts, Amaryllidaceae, dihydroisoquinolinones

\section{Introduction}

Plants from the Amaryllidaceae family are spread all over the world and due to its pharmacological relevance some representatives of this botanic family are known by humans since antiquity. ${ }^{1}$

Among the chemical constituents present in these plants, the alkaloids are the most important and, normally, they are responsible for the biological activities exhibited. As a matter of fact, the biological importance of the plants from this family led to an intense phytochemical research activity, which culminated with the isolation and chemical characterization of several structurally different classes of alkaloids. $^{2}$

Galanthamine (1), ${ }^{3}$ pancratistatin $(2),{ }^{4}$ narciclasine (3), ${ }^{5}$ hippadine (4), ${ }^{6}$ anhydrolycorinone (5), ${ }^{6,7}$ and plicamine $(6)^{8}$ are examples that clearly show the structurally rich diversity of these alkaloids (Figure 1). Besides structural complexity, these alkaloids exhibit different biological activities. Galantamine (1), for example, is a specific, competitive and reversible acetyl cholinesterase inhibitor, being used in the clinical

*e-mail: coelho@iqm.unicamp.br

In memorium of Prof. Helena Ferraz for the outstanding contributions she gave to the Brazilian chemical community. treatment of Alzheimer disease. ${ }^{3}$ Pancratistatin (2), narciclasine (3), hippadine (4) and anhydrolycorinone (5) exhibit antiproliferative activity. ${ }^{9,10}$

The highly sophisticated substitution pattern of the carbon skeleton of these alkaloids, associated with their relevant biological and pharmacological significance, induced the interest of synthetic organic chemists in establishing strategies aiming at their total syntheses, in both racemic and asymmetric versions. The results of these efforts can be easily measured by the countless reports available in the literature describing successful syntheses of several types of alkaloids isolated from Amaryllidaceae. ${ }^{11}$

Particularly, the alkaloids which are structurally related to pantacristatin (2) were synthesized successfully by using the strategy of joining the two suitably substituted fragments, in a convergent way, as shown in the Scheme 1.

This coupling generates two new bonds $(10 \mathrm{~b}$ and $4 \mathrm{a}$, see numeration in Scheme 1), and introduces functional groups at the proper places for the formation of ring B.

Despite its elegance and efficiency, this synthetic strategy suffers from several drawbacks: occurrence of atropoisomers after the formation of the $\mathrm{C} 10$ bond renders completion of the synthesis troublesome $;{ }^{12}$ difficulties in carrying out a systematic study on the structure-activity 
<smiles>COc1ccc(CN(C)C)c2c1OC1CC(O)C=CC21</smiles>

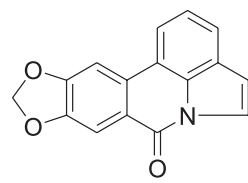

4

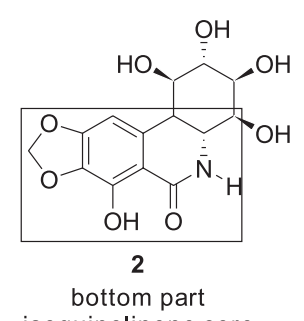

isoquinolinone core

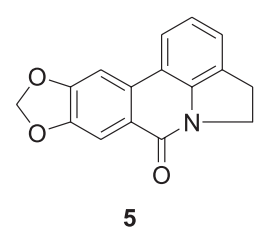<smiles>O=C1NC2C(=C[C@H](O)C(O)C2O)c2cc3c(c(O)c21)OCO3</smiles>

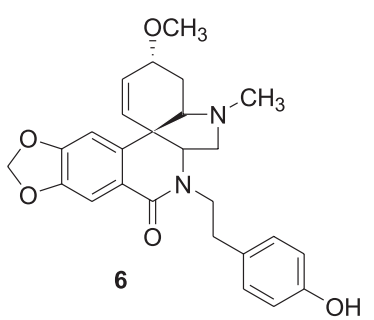

Figure 1. Some representatives examples of Amaryllidaceae alkaloids.

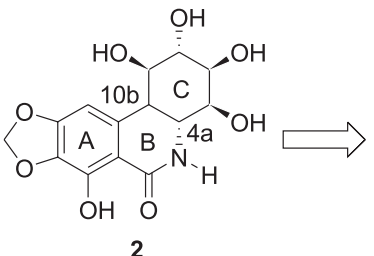

2<smiles>[R]OC(=O)Nc1cc(O)c2c(c1)OCO2</smiles>

7

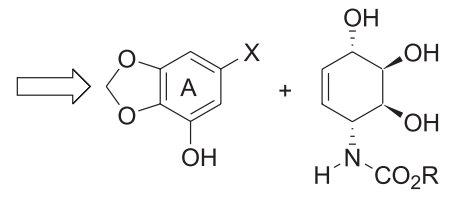

$\begin{array}{cc}\text { Aryl } & \text { sugar like } \\ \text { fragment (8) } & \text { fragment (9) }\end{array}$

Scheme 1. Brief general strategy for the synthesis of some Amaryllidaceae alkaloids.

relationships since the coupling fragments provide advanced intermediates of a great resemblance with the target alkaloid itself.

In an ongoing research program directed to the utilization of Morita-Baylis-Hillman adducts (MBH) as versatile starting materials for the synthesis of different classes of natural and non-natural products ${ }^{13}$ we envisaged developing an alternative strategy to prepare alkaloids from Amaryllidaceae, mostly those structurally related to plicamine (6). ${ }^{14,15}$ Our interest was focused on the establishment of a linear synthetic strategy that should fulfill two basic requirements. Firstly, it should use a simple set of reactions and a readily available starting material, in order to be easily scaled up. Secondly, the alkaloid carbon skeleton should be constructed step by step, thus permitting us to carry out, in a subsequent phase, a structure-activity relationship study of some synthetic intermediates.

From our point of view, the carbon skeleton of the alkaloids in which we were particularly interested (e.g., plicamine (6) and derivatives), should be prepared by a schematic sequence as shown in Scheme 2.

The synthesis of alkaloid skeletons could be accomplished from an intermediate such as $\mathbf{1 0}$, which could be used in the preparation of the skeleton of plicamine (6) through a spiroannelation protocol as previously described by Martin et al. ${ }^{15}$ and Kane et al. ${ }^{16}$ for the synthesis of alkaloids structurally related to it. The synthesis of dihydroisoquinolin$5(6 H)$-one derivative $\mathbf{1 1}$ could be secured from the carbamate 12, which in turn should be prepared from Morita-BaylisHillman adducts.

As part of a study directed towards to the total synthesis of alkaloids from Amaryllidaceae, we describe herein a straightforward total synthesis of the racemic dihydroisoquinolone 11, which we consider an important advanced intermediate in our approach towards the preparation of Amaryllidaceae alkaloids.

\section{Results and Discussion}

We started our sequence with the Morita-BaylisHillman reaction ${ }^{17,18}$ between piperonal and methyl acrylate, in the presence of ultrasound, ${ }^{19}$ which gave the adducts 13 , in $73 \%$ yield. In the next step of our sequence, the methyl ester group of $\mathbf{1 3}$ was chemoselectively reduced. Searching to shorten the synthetic sequence and avoid unnecessary protective steps, we first attempted to run this reduction directly on $\mathrm{MBH}$ adducts using DIBAL-H. However the diol 14 was afforded in only $40 \%$ yield. This low yield was promptly overcome by protecting the secondary hydroxyl group as a silyl ether (tertbutyldimethylsilyl, TBS) ether 15. Subsequent reduction 


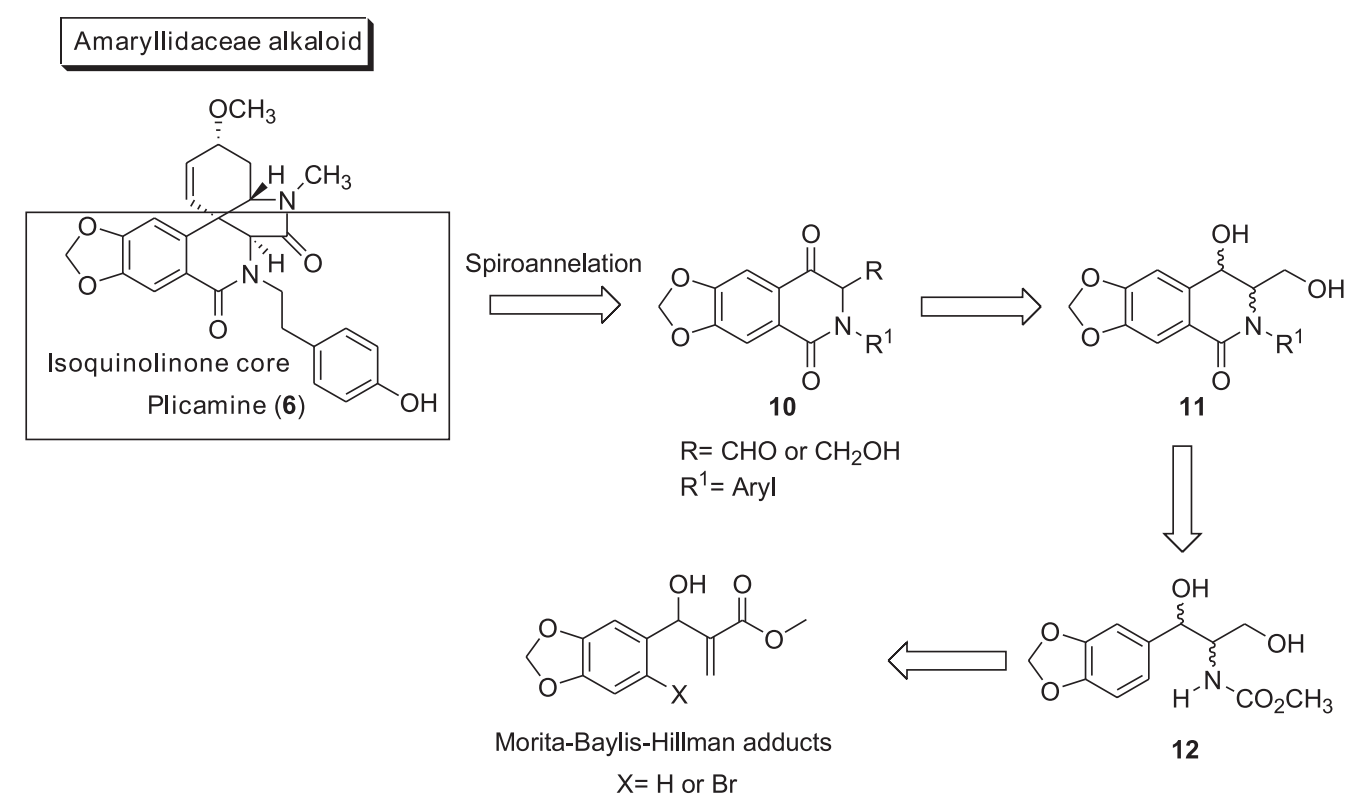

Scheme 2. Alternative linear strategy for the synthesis of Amaryllidaceae alkaloids.

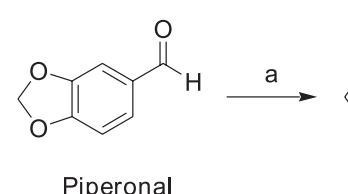

Piperonal<smiles>C=C(C(=O)OC)C(O)c1ccc2c(c1)OCO2</smiles>

13
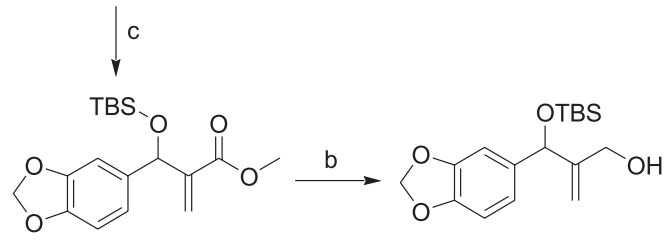

15<smiles>C=C(CO)C(O)c1ccc2c(c1)OCO2</smiles>

16

Reagents and conditions: a) Methyl acrylate, DABCO, $\mathrm{MeOH}$, ultrasound, r.t., $96 \mathrm{~h}, 73 \%$; b) DIBAL-H, $\mathrm{CH}_{2} \mathrm{Cl}_{2},-78{ }^{\circ} \mathrm{C}, 2 \mathrm{~h}$, MBH adduct: $40 \%$; TBS derivative: $98 \%$; c) TBSOTf, $\mathrm{Et}_{3} \mathrm{~N}, \mathrm{CH}_{2} \mathrm{Cl}_{2}, 1$ h, r. t., $96 \%$.

Scheme 3. Preparation of 16.

of 15 with DIBAL-H provided the allyl derivative 16, in an overall yield of $86 \%$ (for two steps) (Scheme 3).

The preparation of the lactam ring present in alkaloid structures could be readily secured by an intramolecular acylation reaction using a carbamate like $\mathbf{1 2}$ as acylating agent (see Scheme 2). This carbamate was readily prepared from 16 following the sequence shown in Schemes 4 and 5 .

The synthesis of keto-amide $\mathbf{1 0}$ (see Scheme 2) requires the selective removal of the protecting group from the secondary hydroxyl group. At this stage of the work, it was important to differentiate the protecting group of the secondary hydroxyl group from that used to protecting the primary hydroxyl group. Thus, allyl diol 16 was treated with triisopropylsilyl triflate in the presence of $\mathrm{CH}_{2} \mathrm{Cl}_{2}$ /DMAP to give the silyl ether 17 in $92 \%$ yield. Subsequent hydroboration of the double bond of $\mathbf{1 7}$ with 9-BBN gave alcohols $\mathbf{1 8 a} / \mathbf{b}$ in $76 \%$ yield, as a mixture of diastereoisomers, in which the syn is the major one (18a, Scheme 4). ${ }^{13}$ The unprotected hydroxyl of the diastereoisomeric mixture was oxidized to the carboxylic acid $\mathbf{2 0}$ in two steps. All attempts to oxidize the alcohol 18a/b directly to the corresponding acid $20 \mathrm{a} / \mathbf{b}$ failed..$^{13}$ We observed an extensive degradation of $\mathbf{1 8 a} / \mathbf{b}$ under several different experimental conditions. Thus the alcohols $\mathbf{1 8} \mathbf{a} / \mathbf{b}$ were treated with TPAP in the presence of NMO to furnish the corresponding aldehyde $19 \mathbf{a} / \mathbf{b}$, in $96 \%$ yield. $^{20}$ Oxidation of the aldehydes with sodium chlorite provided the acid $\mathbf{2 0 a} / \mathbf{b}$, in $90 \%$ yield (Scheme 4 ). ${ }^{13,21}$ After chromatographic purification the minor diastereoisomer (20b) was no longer detected.

Acid 20a was then submitted to a Curtius rearrangement in order to incorporate the nitrogen atom of the 

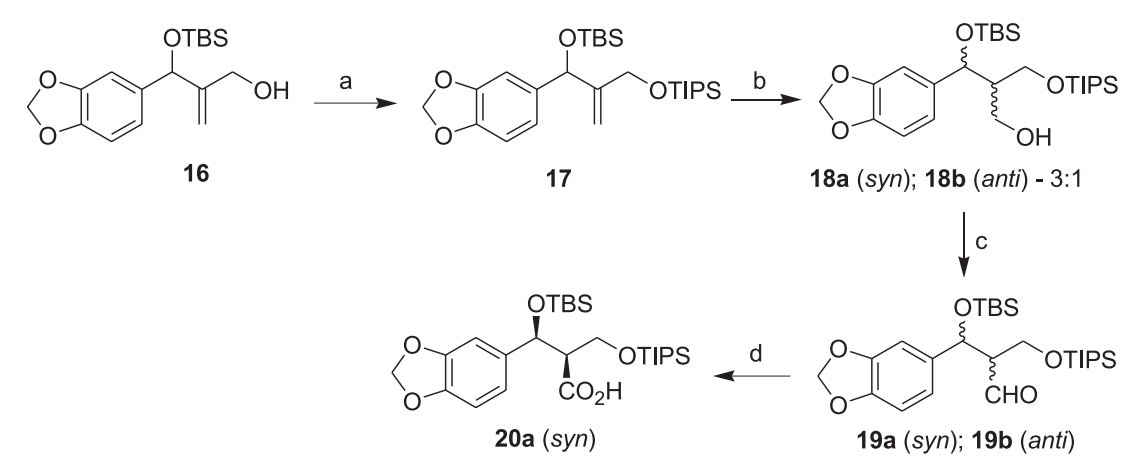

Reagents and conditions: a) TIPSOTf, $\mathrm{Et}_{3} \mathrm{~N}, \mathrm{CH}_{2} \mathrm{Cl}_{2}$, r.t., $1 \mathrm{~h}, 92 \%$; b) $9-\mathrm{BBN}$, THF, $0{ }^{\circ} \mathrm{C} \rightarrow$ r.t., $16 \mathrm{~h}$, then NaOH $3 \mathrm{~mol} \mathrm{~L}{ }^{-1}$, $\mathrm{H}_{2} \mathrm{O}_{2} 30 \%, 0{ }^{\circ} \mathrm{C} \rightarrow$ r. t., 2 h, $75 \%$; c) TPAP, NMO, $\mathrm{CH}_{2} \mathrm{Cl}_{2}$, MS $4 \AA$, 30 min, r. t., $96 \%$; d) $\mathrm{NaO}_{2} \mathrm{Cl}_{,} \mathrm{NaH}_{2} \mathrm{PO}_{4}, t-\mathrm{BuOH}_{\text {, }}$

2-methyl-2-butene, $16 \mathrm{~h}, 0^{\circ} \mathrm{C} \rightarrow$ r. t., $90 \%$.

Scheme 4. Stereoselective preparation of acid 20a.

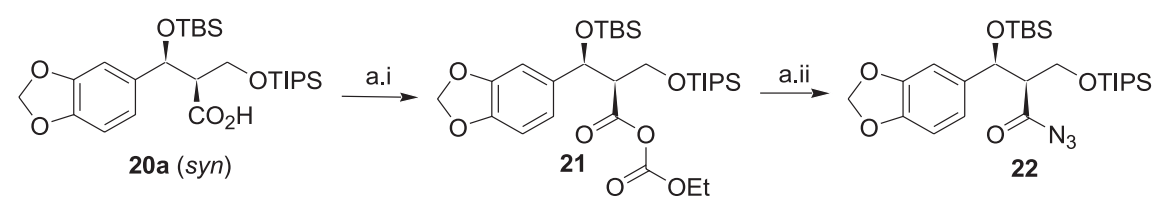

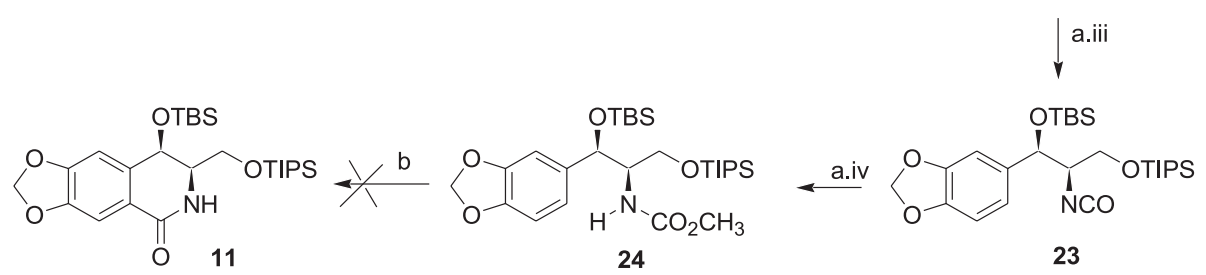

Reagents and conditions: Curtius rearrangement a) i. $\mathrm{ClCO}_{2} \mathrm{Et}, \mathrm{Et}_{3} \mathrm{~N}, 0{ }^{\circ} \mathrm{C}, 40$ min.; ii. $\mathrm{NaN}_{3}, 0{ }^{\circ} \mathrm{C}, 2 \mathrm{~h}$; iii. toluene, reflux, 2h; iv. $\mathrm{MeOH}$, reflux, $12 \mathrm{~h}, 65 \%$ (four steps-one pot sequence); b) $\mathrm{Tf}_{2} \mathrm{O}, \mathrm{DMAP}, \mathrm{CH}_{2} \mathrm{Cl}_{2}, 0^{\circ} \mathrm{C} \rightarrow$ r.t., $2 \mathrm{~h}$.

Scheme 5. Stereoselective preparation of carbamate 24 and cyclization to 11.

lactam unit present in the skeleton of the Amaryllidaceae alkaloids. ${ }^{22}$ The rearrangement was carried out in a one pot sequence to furnish the carbamate 24. Treatment of 20a successively with ethyl chloroformate and sodium azide gave the acylazide intermediate $\mathbf{2 2}$, which was rearranged to the corresponding isocyanate $\mathbf{2 3}$ (not isolated) by refluxing in toluene. After evaporation of the toluene, $\mathbf{2 3}$ was treated with methanol to furnish the carbamate 24, with an overall yield of $65 \%$ from the acid 20a (4 steps) (Scheme 5). ${ }^{23}$

With the carbamate $\mathbf{2 4}$ in our hands, we promoted the internal acylation. To drive this reaction in the direction of the protected dihydroisoquinolin-5(6H)-one (11), carbamate $\mathbf{2 4}$ was treated under several different BischlerNapieralski experimental protocols (triflic anhydride in the presence of DMAP, ${ }^{24} \mathrm{POCl}_{3} /$ py, toluene, ${ }^{25} \mathrm{P}_{2} \mathrm{O}_{5} / \mathrm{POCl}_{3}{ }^{25}$ etc.). However we were unable to detect the formation of compound 11 (Scheme 5).

In all trials we observed either the degradation of the starting materials or its almost total recuperation. The degradation could be related to the lability of the silyl group in the Bischler-Napieralski protocols. During the synthesis of some Licoridine derivatives, McNulty and $\mathrm{Mo}^{26}$ have already described the unsucessfull cyclization of some methoxycarbamates, using Banwell's method, ${ }^{25}$ when a secondary hydroxyl group was protected as a TBS ether. To circumvent this problem, we changed our synthetic strategy. Instead of a silyl ether, the secondary hydroxyl of the MoritaBaylis-Hillman adduct $\mathbf{1 3}$ was now protected as a PMB ether (25) and a more acid-resistant silyl protecting group was chosen to protect the primary hydroxyl group. Using the same reaction sequence described above, PMB-ether $\mathbf{2 5}$ was transformed into the carbamate $\mathbf{3 1}$. When we tried to perform the Bischler-Napieralski reaction with 31 we were able to isolate either the oxazolidin-2-one $\mathbf{3 2}$ or isoquinolol $\mathbf{3 3}$, in 30 and $37 \%$ yields, respectively. Compound $\mathbf{3 3}$ was afforded after removal of the silylated protecting group presents on primary hydroxyl group. No trace of the required isoquinolinone $\mathbf{1 1}$ was detected in either reaction (Scheme 6). 

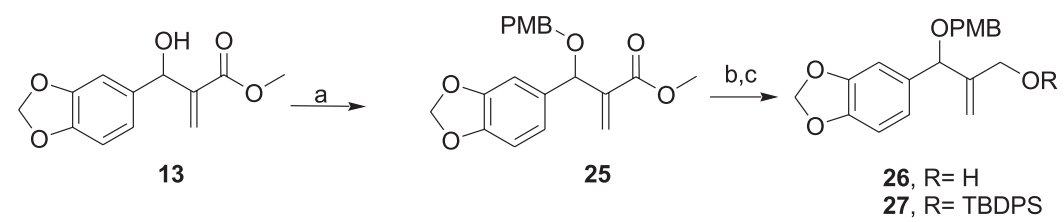

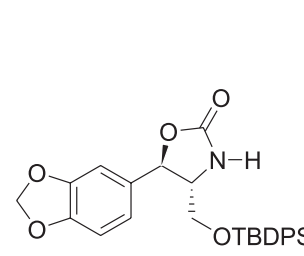

32

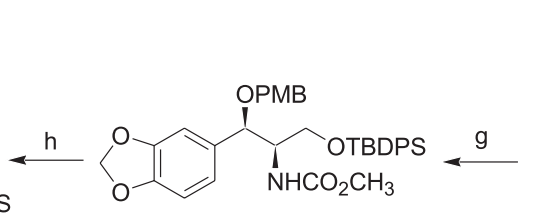

31

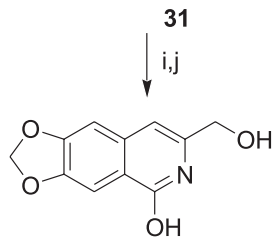

33

Reagents and conditions: a) $\mathrm{Cl}_{3} \mathrm{NHOPMB}, \mathrm{CH}_{2} \mathrm{Cl}_{2}$, CSA, r.t., $18 \mathrm{~h}, 93 \%$; b) DIBAL-H, $\mathrm{CH}_{2} \mathrm{Cl}_{2},-78^{\circ} \mathrm{C}, 2 \mathrm{~h}, 72 \%$; c)TBDPSCl, imidazole, DMF, r.t., $16 \mathrm{~h}, 98 \%$; d) 9-BBN, THF, $0{ }^{\circ} \mathrm{C} \rightarrow$ r.t., $16 \mathrm{~h}$, then $\mathrm{NaOH} 3 \mathrm{~mol} \mathrm{~L}^{-1}, \mathrm{H}_{2} \mathrm{O}_{2} 30 \%, 0{ }^{\circ} \mathrm{C} \rightarrow$ r.t., $2 \mathrm{~h}, 67 \%$; e) TPAP, NMO, $\mathrm{CH}_{2} \mathrm{Cl}_{2}$, MS $4 \AA$, r.t., $1 \mathrm{~h}, 80 \%$; f) $\mathrm{NaO}_{2} \mathrm{Cl}, \mathrm{NaH}_{2} \mathrm{PO}_{4}, t$ - $\mathrm{BuOH}, 2$-methyl-2-buten, $1 \mathrm{~h}, 0^{\circ} \mathrm{C} \rightarrow$ r.t., $88 \%$; g) i. $\mathrm{ClCO}_{2} \mathrm{Et}, \mathrm{Et}_{3} \mathrm{~N}, 0{ }^{\circ} \mathrm{C}, 40 \mathrm{~min}$; ii) $\mathrm{NaN}_{3}, 0{ }^{\circ} \mathrm{C}, 2 \mathrm{~h}$; iii) toluene, reflux, $2 \mathrm{~h}$; iv. $\mathrm{MeOH}$, reflux, $12 \mathrm{~h}, 60 \%$ overall yield (4 steps); h) $\mathrm{Tf}_{2} \mathrm{O}$, DMAP, $\mathrm{CH}_{2} \mathrm{Cl}_{2}, 0{ }^{\circ} \mathrm{C} \rightarrow$ r.t., $2 \mathrm{~h}, 34 \%$; i) $\mathrm{POCl}_{3}$, py, toluene, reflux, $16 \mathrm{~h}, 50 \%$; j) $\mathrm{TBAF}, \mathrm{THF}, 0{ }^{\circ} \mathrm{C}$ $\rightarrow$ r.t., $75 \%$.

Scheme 6. Preparation of $\mathbf{3 2}$ and $\mathbf{3 3 .}$

During development of the asymmetric total synthesis of (+)-pantacristatin, Trost and Pulley ${ }^{27}$ found similar problems when they tried to prepare the isoquinolinone ring of the alkaloid. To solve this problem, bromine was incorporated into the aromatic ring and this was then used as substrate for a halogen-lithium exchange. The aryl lithium compound generated in situ participated in an intramolecular nucleophilic attack on the isocyanate to furnish the required isoquinolinone ring in a good yield (Figure 2).

This simple idea inspired us and we again started our sequence, now using a brominated derivative of piperonal as starting material. The Morita-Baylis-Hillman reaction between 6-bromopiperonal and methyl acrylate in the presence of ultrasound and a catalytic quantity of an ionic liquid (1-methyl methylimidazolium, [bmin] $\mathrm{PF}_{6}$ ) furnished 34 in $80 \%$ yield, as the sole product. ${ }^{28,29}$ At this stage of the work and having the results of the sequence with piperonal in mind, we decided to prepare some derivatives in which the difference among them was only concerning the groups used for protecting the primary and secondary hydroxyl groups. Our interest in doing this was to evaluate which combination of protecting groups would be the most adequate for the cyclization step. Adduct $\mathbf{3 4}$ was then treated with TBSOTf or TIPSOTf to give the corresponding silyl ethers $\mathbf{3 5}$ and 36, in 98 and $87 \%$ yields, respectively. Both silylated compounds were reduced with DIBAL-H, at $-78{ }^{\circ} \mathrm{C}$ to provide the mono-silylated alcohols 37 and $\mathbf{3 8}$ in excellent chemical yields (97 and 95\%, respectively). Compounds $\mathbf{3 7}$ and $\mathbf{3 8}$ were both submitted to another protecting step. Compound $\mathbf{3 7}$ was treated with TBDPSCl and imidazole to give 39 in $93 \%$ yield. Two different protecting groups were introduced in the primary hydroxyl group of $\mathbf{3 8}$. First, $\mathbf{3 8}$ was allowed to react with PMB trichloroimidate in dichloromethane to furnish compound $\mathbf{4 0}$, in $98 \%$ yield. The compound $\mathbf{3 8}$ was also treated with TBDPSCl and imidazole to give the compound $\mathbf{4 1}$, in $93 \%$ yield. Hydroboration reactions of compounds $\mathbf{3 9}, \mathbf{4 0}$ and $\mathbf{4 1}$ gave the alcohols $\mathbf{4 2 ,} \mathbf{4 3}$ and $\mathbf{4 4}$, in 82,76 and $\mathbf{7 7 \%}$ yields, respectively. These alcohols were used as substrates

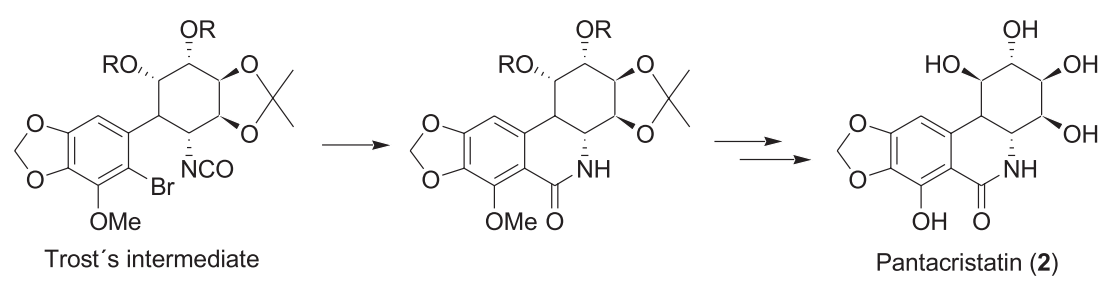

Figure 2. Trost's synthetic strategy for the asymmetric synthesis of pantacristatin. 
for the oxidation steps. First, alcohols 42-44 were reacted with TPAP to give the aldehydes $\mathbf{4 5 ,} 46$ and $\mathbf{4 7}$ in 98, 85 and $95 \%$ yields, respectively. Finally, the aldehydes $45-$ 47 were oxidized according to Pinnick conditions ${ }^{21}$ to give acids 48, 49 and 50, in 92, 80 and 85\% yields (Scheme 7).

The acids 48-50 were easily prepared in six steps with overall yields of 66, 42 and 54\%, respectively. Following our sequence the three acids were submitted to the Curtius rearrangement. To our delight, the treatment of the isocyanate intermediates with $t$-BuLi in diethyl ether gave the required dihydroisoquinolinone 11, in good overall yield (Scheme 8).

A careful spectroscopic analysis of the data recorded for 11 confirmed the proposed structure of this intermediate. At this stage, we have accomplished the syntheses of the isoquinolinone skeleton (51-53) in 8 steps with overall yields of 25, 20 and 16\% from the Morita-BaylisHillman adduct 34. The next step of our sequence would be $\mathrm{N}$-alkylation to provide the required bottom part of the plicamine skeleton. To carry out this alkylation reaction,<smiles>[R2]C(C(=C)CO)c1cc2c(cc1Br)OCO2</smiles><smiles>[R]CC(C=O)C([R])c1cc2c(cc1Br)OCO2</smiles>

45, $R=T B S ; R^{1}=$ TBDPS 46, $R=T I P S ; R^{1}=P M B$ 47, $R=T I P S ; R^{1}=$ TBDPS i i<smiles>[R]CC(C(=O)O)C([R])c1cc2c(cc1Br)OCO2</smiles>

48, $R=T B S ; R^{1}=$ TBDPS

49, $R=T I P S ; R^{1}=P M B$

50, $R=T I P S ; R^{1}=$ TBDPS

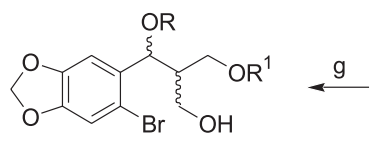

42, $R=T B S ; R^{1}=$ TBDPS, syn : anti $-6: 1$

43, R= TIPS; $\mathrm{R}^{1}=\mathrm{PMB}$, syn : ant $-3: 1$

44, R= TIPS; $\mathrm{R}^{1}=$ TBDPS, syn ; anti $-4: 1$<smiles></smiles>

39, $R=T B S ; R^{1}=$ TBDPS

40, $R=T I P S ; R^{1}=P M B$

41, $R=$ TIPS; $R^{1}=$ TBDPS

Reagents and conditions: a) Methyl acrylate, [bmim] $\left[\mathrm{PF}_{6}\right], \mathrm{MeOH}$, r.t., ultrasound, $96 \mathrm{~h}, 80 \%$; b) TBSOTf, $\mathrm{Et}_{3} \mathrm{~N}, \mathrm{CH}_{2} \mathrm{Cl}_{2}, \mathrm{r}_{\mathrm{t}}$. $1 \mathrm{~h}, 98 \%$ (for 35); c) TIPSOTf, $\mathrm{Et}_{3} \mathrm{~N}, \mathrm{CH}_{2} \mathrm{Cl}_{2}$, r.t., $1 \mathrm{~h}, 87 \%$ (for 36); d) DIBAL-H, $\mathrm{CH}_{2} \mathrm{Cl}_{2},-78{ }^{\circ} \mathrm{C}, 2 \mathrm{~h}, 98 \%(37)$ and $95 \%$ (38); e) TBDPSCl, $\mathrm{Et}_{3} \mathrm{~N}, \mathrm{DMAP}, \mathrm{CH}_{2} \mathrm{Cl}_{2}$, r.t., 16 h, $93 \%$ (for 39) and $91 \%$ (for 41); f) $\mathrm{Cl}_{3} \mathrm{CNHOPMB}_{2} \mathrm{CH}_{2} \mathrm{Cl}_{2}, \mathrm{CSA}$, r.t., $18 \mathrm{~h}$ $98 \%$ (for 40); g) 9-BBN, THF, $0{ }^{\circ} \mathrm{C} \rightarrow$ r.t., $16 \mathrm{~h}$, then $\mathrm{NaOH}, 3 \mathrm{~mol} \mathrm{~L}^{-1}, \mathrm{H}_{2} \mathrm{O}_{2} 30 \%, 0{ }^{\circ} \mathrm{C} \rightarrow$ r.t., $2 \mathrm{~h}, 82 \%$ (for 42 ), $76 \%$ (for 43 ) and 77\% (for 44); h) TPAP, NMO, $\mathrm{CH}_{2} \mathrm{Cl}_{2}$, MS $4 \AA$, r.t., $1 \mathrm{~h}, 98 \%$ (for 45 ), $85 \%$ (for 46 ) and $95 \%$ (for 47 ); i) $\mathrm{NaO}_{2} \mathrm{Cl}, \mathrm{NaH}_{2} \mathrm{PO}_{4}, 2-\mathrm{Methyl}-2$-buten, $0{ }^{\circ} \mathrm{C} \rightarrow$ r.t., $1 \mathrm{~h}, 92 \%$ (for $\mathbf{4 8}$ ), $80 \%$ for $\mathbf{4 9}$ ) and $85 \%$ (for $\mathbf{5 0}$ ).

Scheme 7. Preparation of acids 48-50.<smiles>[R]OCC(C(=O)O)C([R])c1cc2c(cc1Br)OCO2</smiles>

48, $R=T B S ; R^{1}=$ TBDPS 49, $R=$ TIPS; $R^{1}=P M B$ 50, $R=$ TIPS; $R^{1}=$ TBDPS<smiles>[R]OCC(COCC)C([R])c1cc2c(cc1Br)OCCO2</smiles>

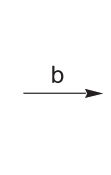

51, $R=T B S ; R^{1}=$ TBDPS 52, $R=T I P S ; R^{1}=P M B$ 53, $R=$ TIPS; $R^{1}=$ TBDPS

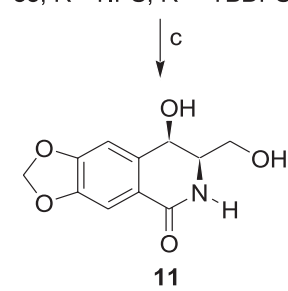


the alkylating agent need to be prepared from commercial 4-hydroxy-phenyl acetic acid (54). Thus, acid 54 was first transformed to the corresponding ethyl ester $\mathbf{5 5}$, followed by protection of the phenolic hydroxyl group with TIPSOTf or methyl iodide to furnish the silylated ester $\mathbf{5 6}$ or the methoxy derivative 57. Reduction of ester furnished the homobenzylic alcohols $\mathbf{5 8}$ and $\mathbf{5 9}$, which were converted to the aryl bromides $\mathbf{6 0}$ and $\mathbf{6 1}$, in 4 steps with an overall yield of $77 \%$ (Scheme 9).

All spectroscopic data of $\mathbf{6 0}$ and $\mathbf{6 1}$ are compatible with the proposed structures. Isoquinolinone $\mathbf{5 1}$ was then treated with $\mathrm{NaH}$ at room temperature, followed by the addition of the bromide $\mathbf{6 0}$. To our surprise, no N-alkylated product was detected in the reaction medium, even after $24 \mathrm{~h}$. After isolation, the only recovered product was the starting material 51. To solve this problem, we tried different experimental conditions; unfortunately, no Nalkylated product was detected.

Trying to surmount this problem, we changed the alkylating agent. Instead of aryl bromide an aryl iodide was used, ${ }^{30}$ however no $\mathrm{N}$-alkylation product was detected even when a more efficient alkylating agent was employed.

During the development of this work, Ley and coworkers ${ }^{14}$ described the first asymmetric total synthesis of plicamine (6). These authors also had the same problem for alkylating the nitrogen atom of the isoquinolinone ring. This problem was solved by reducing the isoquinolinone to the corresponding tetrahydroquinoline, which was now sucessfully alkylated in good yield. Most probably, the electron pair of this nitrogen is in resonance with the carbonyl group and with the aromatic ring, which compromise its nucleophilicity. This is confirmed by the fact that when the carbonyl group was removed the $\mathrm{N}$ alkylation reaction worked very well.

Owing to this problem in the last step of our sequence, we decided to consider a synthetic alternative to prepare the required advanced intermediate 11. From our point of view, the $\mathrm{N}$-alkylation step could be carried out in the middle of the sequence and the cyclization step could then be effected as the last step, with an already $\mathrm{N}$-alkylated intermediate. To do this it was necessary to have a modification during the Curtius rearrangement in order to produce an amine instead of a carbamate.

Acid 48 was again submitted to Curtius rearrangement conditions, however now the isocyanate intermediate was allowed to react with $\mathrm{NaOH}$ in $\mathrm{THF}$ to produce the required amine 62, as depicted in Scheme 10, with an overall yield of $62 \%$.

Before starting the $\mathrm{N}$-alkylation reaction with amine 62, we altered the structure of the alkylating agent in order to have good selectivity when the removal of the protecting group was necessary (see Scheme 9). Then, ester 55 was treated with MeI in acetone to furnish the O-methoxy ethyl ester 57, which was reduced with DIBAL-H to give alcohol 59. Bromide $\mathbf{6 1}$ was prepared from alcohol 59 by treatment with $\mathrm{CBr}_{4} / \mathrm{PPh}_{3}$, in 3 steps from ester 55, in $84 \%$ overall yield.

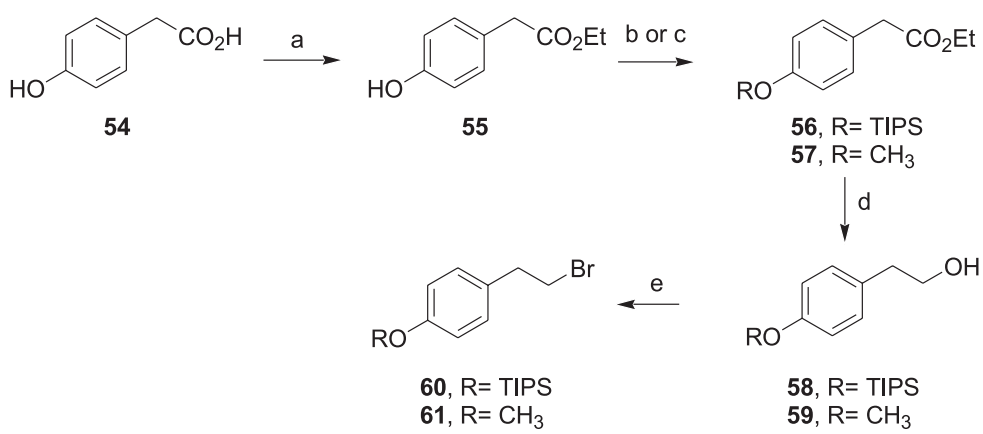

Reagents and conditions: a) EtOH, toluene, $p$-TsOH (cat.), reflux, 16 h, $>99 \%$; b) TIPSOTf, $\mathrm{Et}_{3} \mathrm{~N}, \mathrm{CH}_{2} \mathrm{Cl}_{2}, 1$ h, r.t., $92 \%$; c) MeI, acetone, $\mathrm{Et}_{3} \mathrm{~N}, 6{ }^{\circ} \mathrm{C}, 8 \mathrm{~h}, 95 \%$; d) DIBAL-H, $\mathrm{CH}_{2} \mathrm{Cl}_{2},-78{ }^{\circ} \mathrm{C}, 1 \mathrm{~h}, 88 \%$ (for 58) and $90 \%$ (for 59); e) $\mathrm{CBr}_{4}, \mathrm{Ph}_{3} \mathrm{P}$, $\mathrm{CH}_{3} \mathrm{CN}, 2,6$-lutidine, $15 \mathrm{~min}$., $96 \%$ (for 60 ) and $>99 \%$ (for 61 ).

Scheme 9. Preparation of alkylating agent 60 or 61.

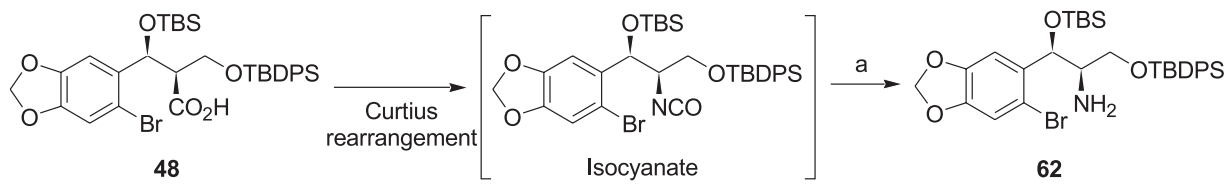

Reagents and conditions: a) $\mathrm{NaOH} 1.5 \mathrm{~mol} \mathrm{~L}^{-1}$, THF, $0{ }^{\circ} \mathrm{C} \rightarrow 70{ }^{\circ} \mathrm{C}, 4 \mathrm{~h}, 62 \%$ overall yield.

Scheme 10. Preparation of amine 62. 
Then amine 62 was allowed to react with $\mathrm{Na}_{2} \mathrm{CO}_{3}$ in acetonitrile under reflux, followed by the addition of the bromide $\mathbf{6 1}$ to smoothly provide the $\mathrm{N}$-alkylated product $\mathbf{6 3}$, in $85 \%$ yield. The secondary amine $\mathbf{6 3}$ was then treated with methyl chloroformate to give carbamate $\mathbf{6 4}$, in $90 \%$ yield. Finally, the treatment of $\mathbf{6 4}$ with $t$-BuLi in THF at $-78^{\circ} \mathrm{C}$ afforded the $\mathrm{N}$-alkylated isoquinolinone 65 , in $60 \%$ yield (Scheme 11).

Based on this modification, isoquinolinone 65 was prepared in 10 steps with an overall yield of $19 \%$. To achieve our target, the TBS protecting group should be removed. To our surprise, selective removal of this group was revealed to be particularly troublesome, independent of the experimental conditions employed. Trying to finding the best way to selectively removed one of the silylated groups, we have carried out some experiments with different conditions, using as substrate some isoquinolinones prepared during the development of our synthetic study (51-53, see Scheme 8). The results achieved are summarized in Table 1.

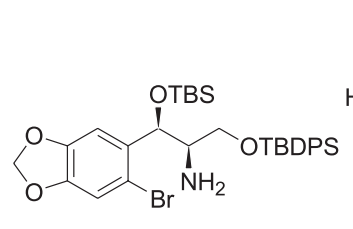

62<smiles>COc1ccc(CCBr)cc1</smiles><smiles>COc1ccc(CCNC(CO[Pb])C([O+])c2cc3c(cc2Br)OCO3)cc1</smiles><smiles>[3H][Te]</smiles><smiles>CCOCC1C(O[Sb](C)(C)(=O)O)c2cc3c(cc2C(=O)N1CCc1ccc(OC)cc1)OCO3</smiles><smiles>COCCC(C(O)c1cc2c(cc1Br)OCO2)N(CCOC(=O)OC)C(=O)OCc1ccc(OC)cc1</smiles>

Reagents and conditions: a) $\mathrm{Na}_{2} \mathrm{CO}_{3}, \mathrm{CH}_{3} \mathrm{CN}$, reflux, $24 \mathrm{~h}, 85 \%$; b) $\mathrm{ClCO}_{2} \mathrm{Me}, \mathrm{Et}_{3} \mathrm{~N}$, acetone, $0{ }^{\circ} \mathrm{C}, 40$ min., $90 \%$; c) $t$ - $\mathrm{BuLi}$, THF, $-78^{\circ} \mathrm{C}, 1 \mathrm{~h}, 60 \%$.

Scheme 11. Synthesis of the functionalised isoquinolinone $\mathbf{6 5}$.

Table 1. Attempts to selectively remove a secondary protecting group

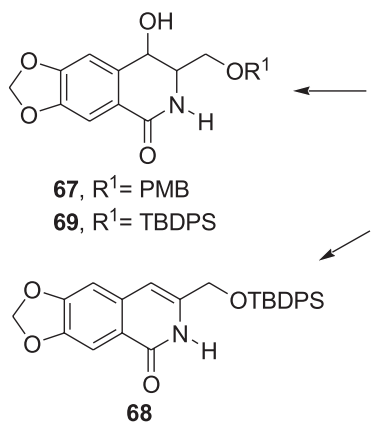<smiles>[R]OC[C@H]1NC(=O)c2cc3c(cc2C1O[13CH])OCO3</smiles>

51, $R=T B S ; R^{1}=T B D P S$<smiles>CCC1Oc2cc3c(cc2O1)C(O)C(CO)NC3=O</smiles>

52, $R=T I P S ; R^{1}=P M B$

53, $R=$ TIPS; $R^{1}=$ TBDPS<smiles>O=C1NC(CO)C(OC(F)(F)F)c2cc3c(cc21)OCO3</smiles>

66

\begin{tabular}{|c|c|c|c|c|}
\hline Entry & Substrate & Experimental conditions & Product & Yield / \% \\
\hline 1 & 52 & HF/py/THF/r.t. & 66 & 45 \\
\hline 2 & 52 & HF/py/THF/reflux & $-{ }^{\mathrm{a}}$ & - \\
\hline 3 & 51 and 53 & $\mathrm{TBAF} / \mathrm{THF} / 0^{\circ} \mathrm{C}$ & 11 & 75 \\
\hline 4 & 52 & $\mathrm{TBAF} / \mathrm{THF} / 0^{\circ} \mathrm{C}$ & 67 & 22 \\
\hline 5 & 51 & $\mathrm{p}-\mathrm{TsOH} /$ benzene $/ 50^{\circ} \mathrm{C}$ & 68 & 70 \\
\hline 6 & 53 & $\mathrm{HCl} 3 \mathrm{~mol} \mathrm{~L}^{-1} / \mathrm{r} . \mathrm{t}$. & $-{ }^{\mathrm{a}}$ & - \\
\hline 7 & 51 and 53 & $\mathrm{HCl} 6 \mathrm{~mol} \mathrm{~L}^{-1} /$ r.t. & 11 & 52 \\
\hline 8 & 51 & $\mathrm{HCl} 3 \mathrm{~mol} \mathrm{~L}^{-1} /$ r.t. & 69 & 20 \\
\hline 9 & 53 & $\mathrm{AcOH} /$ r.t. & $-b$ & \\
\hline
\end{tabular}

${ }^{a}$ an extensive degradation of the substrate was observed; bthe substrate was entirely recovered. 
For most of the cases, no selectivity was observed. For one case, the protecting group of the primary hydroxyl was surprisingly removed faster than that attached on the secondary hydroxyl (entry 1). Under three different experimental conditions it was possible to selectively removed the secondary protecting group, as required, however in two of them the yields are really low (entries 4 and 8), in the third case an elimination reaction occurred (entry 5). Degradation of starting material as well as its recovery was also observed under some experimental conditions (entries 2, 6 and 9, respectively)

These results obliged us to change our initial sequence and to add an unexpected step. Thus, isoquinolinone $\mathbf{6 5}$ was treated with TBAF in anhydrous THF at room temperature to provide diol $\mathbf{7 0}$, in $75 \%$ yield. Now, the primary hydroxyl group of diol $\mathbf{7 0}$ was selectivity protected to give $\mathbf{7 1}$, in $80 \%$ yield, as the sole detectable product (Scheme 12).

The structure of isoquinolinone $\mathbf{7 0}$ sucessfully incorporates all the groups needed to accomplish the preparation of the skeleton of Amaryllidaceae alkaloids. The highly functionalized isoquinolinone $\mathbf{7 0}$ was synthesized in 9 steps with an overall yield of $11 \%$ from Morita-Baylis-Hillman adduct $\mathbf{3 4}$.

In summary, this strategy has permitted us to prepare an advanced intermediate which could be used in a racemic or asymmetric total synthesis of Amaryllidaceae alkaloids. The structure of isoquinolinone $\mathbf{7 0}$ exhibits all substituents that are found on the bottom part of the structure of alkaloid plicamine (6). Studies to accomplish the total synthesis of an Amaryllidaceae alkaloid through this strategy are ongoing in our laboratory and will be disclosed in due time.

\section{Experimental}

The ${ }^{1} \mathrm{H}$ and ${ }^{13} \mathrm{C}$ spectra were recorded on a Varian GEMINI BB-300 at $300 \mathrm{MHz}$ and $75.4 \mathrm{MHz}$, respectively, or on an Inova instrument at $500 \mathrm{MHz}$ and $125 \mathrm{MHz}$, respectively. The mass spectra were recorded using a HP 5988A GC/MS with a High Resolution AutospecMicromass/EBE. IR were obtained with a Nicolet model
Impact 410. Melting points were measured in open capillary tubes using an Electrothermal model 9100 apparatus, and are uncorrected. Yields were determined from GC analyses on a HP6890 equipment with a flame ionization detector, using a HP-5 capillary (crosslinked $5 \%$ phenylmetylsiloxane, $28 \mathrm{~m}$ ) column. Manipulations and reactions were not performed under dry atmospheres or employing dry solvents, unless otherwise specified. Purification and separations by column chromatography were performed on silica gel, using normal or flash chromatography. TLC visualization was achieved by spraying with 5\% ethanolic phosphomolybdic acid and heating. All Baylis-Hillman reactions were sonicated in an ultrasonic cleaner, UNIQUE model GA 1000 (1000 W, $25 \mathrm{kHz}$ ). Aromatic aldehydes were purchased from Aldrich, Acros or Lancaster and were used without previous purification

General procedure for the preparation of Morita-BaylisHillman adducts

A mixture of aromatic aldehyde ( $3 \mathrm{~g}, 18-20 \mathrm{mmol})$, methyl acrylate (14-20 mmol) and DABCO (11-13 mmol) in methanol, dichloromethane or acetonitrile $\left(2 \mathrm{~cm}^{3} \mathrm{mmol}^{-1}\right.$, indicated for each aldehyde), was sonicated for 16-96 hours. The ultrasound bath temperature was constantly monitored and kept at $30-45{ }^{\circ} \mathrm{C}$ during the reaction, through ice addition or by using a refrigerated recirculator. After the reaction time, the mixture was diluted with dichloromethane $(50 \mathrm{~mL})$. The organic solution was washed with $10 \%$ aqueous $\mathrm{HCl}(2 \times 20 \mathrm{~mL})$, concentrated under reduced pressure and dried over $\mathrm{MgSO}_{4}$. After filtration and solvent removal, the residue was filtered through a pad of silica gel (eluent indicated for each adduct).

\section{( \pm -Methyl 2-[1,3-benzodioxol-5-yl(hydroxy)methyl] acrylate (13)}

Reaction time: $96 \mathrm{~h}$, methanol solvent; $73 \%$ of a white solid (yield based on recovered aldehyde), purified by silica gel column chromatography (eluting with hexane/ethyl acetate 75:25); $\mathrm{mp} 40-41{ }^{\circ} \mathrm{C}$; IR $v_{\max } / \mathrm{cm}^{-1}: 3492,3119$,

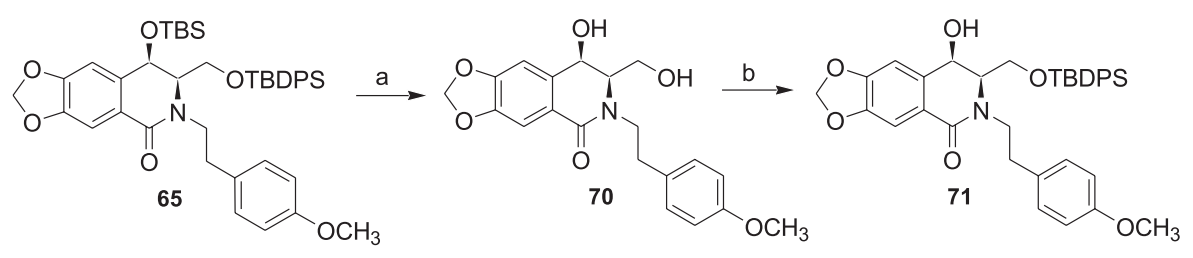

Reagents and conditions: a) TBAF, THF, r.t., 1 h, $75 \%$; b) TBDPSCl, DMAP, $\mathrm{Et}_{3} \mathrm{~N}, \mathrm{CH}_{2} \mathrm{Cl}_{2}$, r.t., 24 h, $80 \%$. 
1706, 1620; ${ }^{1} \mathrm{H}$ NMR $\left(500 \mathrm{MHz}, \mathrm{CDCl}_{3}\right): \delta 2.87$ (bs, 1H, $\mathrm{OH}), 3.78(\mathrm{~s}, 3 \mathrm{H}), 5.45(\mathrm{~s}, 1 \mathrm{H}), 5.82(\mathrm{~s}, 1 \mathrm{H}), 5.87(\mathrm{~s}, 2 \mathrm{H}$, $\left.\mathrm{OCH}_{2} \mathrm{O}\right), 6.3$ (s, 1H), 6.77 (d, $\left.J 8.0 \mathrm{~Hz}, 1 \mathrm{H}\right), 6.84$ (ddd, $J$ $8.0 \mathrm{~Hz}, J 1.8 \mathrm{~Hz}, J 0.5 \mathrm{~Hz}, 1 \mathrm{H}), 6.87$ (dd, $J 1.9 \mathrm{~Hz}$ and $J$ $0.5 \mathrm{~Hz}, 1 \mathrm{H}),{ }^{13} \mathrm{C} \mathrm{NMR}\left(125 \mathrm{MHz}, \mathrm{CDCl}_{3}\right) \delta 51.9,72.9$, $101,107.1,108.1,120.1,125.9,135.2,141.9,147.2$, 147.7, 166.7. MS (70 eV, m/e, \%) $236(\mathrm{M}+, 84), 204$ (30), 176 (27), 151 (40), 149 (100), 93 (55), 65 (44); HRMS (EI, $70 \mathrm{eV}$ ) calc. for $\mathrm{C}_{12} \mathrm{H}_{12} \mathrm{O}_{5}\left[\mathrm{M}^{+}\right]$236.0684. Found: 236.0679. Anal. Calc. for $\mathrm{C}_{12} \mathrm{H}_{12} \mathrm{O}_{5}$ : C, 61.01; H, 5.12\%. Found: C, 60.81; H, 4.93\%.

\section{( \pm -Methyl 2-[(6-bromo-1,3-benzodioxol-5-yl)(hydroxy) methyl]acrylate (34)}

Reaction time: $96 \mathrm{~h}$ methanol solvent; a catalytic amount $(0.03 \mathrm{~mL})$ of 1 -methyl-3-butylimidazolium hexafluorophosphate $\left[(\mathrm{bmim}) \mathrm{PF}_{6}\right]$ was used in this reaction; $80 \%$ yield of a white solid, purified by silica gel column chromatography, eluting with hexane/ethyl acetate, (75:25); mp 101-102 ${ }^{\circ} \mathrm{C}$; IR $v_{\max } / \mathrm{cm}^{-1}: 3483,2954,2920$, 2854, 1720, 1631, 1477, 1261, 1234, 1038; ${ }^{1} \mathrm{H}$ NMR (500 $\left.\mathrm{MHz}, \mathrm{CDCl}_{3}\right): \delta 2.87(\mathrm{bs}, 1 \mathrm{H}, \mathrm{OH}), 3.80(\mathrm{~s}, 3 \mathrm{H}) 5.62(\mathrm{~s}$, $1 \mathrm{H}), 5.82(\mathrm{~s}, 1 \mathrm{H}), 6.01\left(\mathrm{~s}, 2 \mathrm{H}, \mathrm{OCH}_{2} \mathrm{O}\right), 6.26(\mathrm{~s}, 1 \mathrm{H}), 7.0$ (s, $1 \mathrm{H}$ aromatic), 7.02 (s, $1 \mathrm{H}$ aromatic). ${ }^{13} \mathrm{C}$ NMR $(125$ $\left.\mathrm{MHz}, \mathrm{CDCl}_{3}\right) \delta$ 52.1, 71.4, 101.8, 108.2, 112.6, 113.6, $126.8,133.1,140.6,147.6,147.9,166.9$. MS $(70 \mathrm{eV}, \mathrm{m} / \mathrm{z}$, \%) 315(M+2, 17), $313(\mathrm{M}+, 15), 235$ (87), 203 (43), 175 (24), 149 (100), 122 (81), 113 (66), 63 (60); HRMS (EI, $70 \mathrm{eV})$ Calc. for $\mathrm{C}_{12} \mathrm{H}_{11} \mathrm{BrO}_{5}\left[\mathrm{M}^{+}\right]$313.9790. Found: 313.9771 .

(士) Methyl 3-(tert-butyldimethyl-silanyloxy)-3-(1,3-benzodioxol-5-yl)2-methyl propanoate (15)

To a solution of $13(0.5 \mathrm{~g}, 2.12 \mathrm{mmol})$ in $15 \mathrm{~mL}$ of anhydrous dichloromethane was added successively, at room temperature and under an atmosphere of argon, anhydrous triethylamine $(0.6 \mathrm{~mL}, 4.24 \mathrm{mmol})$ and tertbutyldimethylsilyl triflate $(0.52 \mathrm{~mL}, 2.75 \mathrm{mmol})$. The resulting mixture was stirred for $1 \mathrm{~h}$ at room temperature. Then, the reaction was diluted with dichloromethane and the organic layer was washed with a saturated solution of $\mathrm{NaHCO}_{3}(2 \times 50 \mathrm{~mL})$ and brine $(2 \times 50 \mathrm{~mL})$. After separation, the organic phase was dried over anhydrous magnesium sulfate. The solvent was removed under reduced pressure and the residue was purified by flash silica-gel colum chromatography, using as eluent a mixture of hexane/ethyl acetate $2 \%$, to furnish $0.71 \mathrm{~g}$ of silyl ether 15 as a colorless oil in $96 \%$ yield.

IR $v_{\max } / \mathrm{cm}^{-1}: 2954,1723,1631,1488,1443,1247$, 1079; ${ }^{1} \mathrm{H} \mathrm{NMR}\left(500 \mathrm{MHz}, \mathrm{CDCl}_{3}\right): \delta-0.08(\mathrm{~s}, 3 \mathrm{H}), 0.06$ (s, 3H), 0.88 (s, 9H), 3.70 (s, 3H), 5.52 (s, 1H), 5.96 (d, $J$ $\left.1.5 \mathrm{~Hz}, 2 \mathrm{H}, \mathrm{OCH}_{2} \mathrm{O}\right), 6.06$ (bs, 1H), 6.23 (bs, 1H), 6.76.85 (m, 3H aromatics); ${ }^{13} \mathrm{C}$ NMR (125 $\left.\mathrm{MHz}, \mathrm{CDCl}_{3}\right)$ : $\delta-5.1,-4.9,18.1,25.7,51.6,72.3,100.9,107.4,107.7$, $120.6,123.5,136.6,143.9,146.7,147.3,166.3$; HRMS (EI, 70eV) $\mathrm{m} / z$ Calc. for $\mathrm{C}_{18} \mathrm{H}_{26} \mathrm{O}_{5} \mathrm{Si}\left[\mathrm{M}^{+}\right]: 350.1550$. Found: $293.0845\left[\mathrm{M}^{+}\right.$- $t$-butyl].

\section{(土)-2-[1,3-Benzodioxol-5-yl(tert-butyldimetylsilyloxy) methyl]prop-2-en-1-ol (16)}

To a solution of silyl ether $15(0.35 \mathrm{~g}, 1 \mathrm{mmol})$ in anhydrous dichloromethane, at $-78{ }^{\circ} \mathrm{C}$ and under an atmosphere of argon, was added of solution $1.0 \mathrm{~mol} \mathrm{~L}^{-1}$ of DIBAL-H (2.4 mL, $2.4 \mathrm{mmol})$ The reaction mixture was stirred for $2 \mathrm{~h}$ at $-78^{\circ} \mathrm{C}$. After this time, a saturated solution of sodium acetate was added and the reaction mixture was dropped into a mixture of ethyl ether $(50 \mathrm{~mL})$ and a saturated solution of $\mathrm{NH}_{4} \mathrm{Cl}(4.6 \mathrm{~mL})$. The resulting two phase system was stirred for $1 \mathrm{~h}$, which led to formation of a gel that was filtered over a pad of Celite ${ }^{\circledR}$. The solid was washed with ethyl ether $(5 \times 15 \mathrm{~mL})$ and the organic phases were combined, dried over $\mathrm{MgSO}_{4}$ and removed under reduced pressure. The residue was purified by flash silica gel column chromatography to afford alcohol 16, as a colorless viscous oil, in 98\% yield.

IR $v_{\max } / \mathrm{cm}^{-1}: 3389,2955,2929,1655,1487,1441$, 1248, 1041; ${ }^{1} \mathrm{H}$ NMR (300 MHz, $\left.\mathrm{CDCl}_{3}\right): \delta-0.07$ (s, 3H), 0.09 (s, 3H), 0.93 (s, 9H, t-butyl), 1.86 (bs, 1H, OH), 3.85 (d, J 13.5 Hz, 1H), 4.02 (d, J 13.5 Hz, 1H), 5.05 (s, 1H), $5.11(\mathrm{~s}, 1 \mathrm{H}), 5.15(\mathrm{~s}, 1 \mathrm{H}), 5.95\left(\mathrm{~s}, 2 \mathrm{H}, \mathrm{OCH}_{2} \mathrm{O}\right), 6.7-6.8$ (m, 3H aromatics); $\left.{ }^{13} \mathrm{C} \mathrm{NMR} \mathrm{(75} \mathrm{MHz}, \mathrm{CDCl}_{3}\right): \delta-4.8$, $0.2,18.5,26.0,63.4,77.3,101.2,106.9,108.1,112.0$, 119.6, 136.9, 147.0, 147.8, 150.7. HRMS (EI, 70eV) $m / z$ : Calc. for $\mathrm{C}_{17} \mathrm{H}_{26} \mathrm{O}_{4} \mathrm{Si}\left[\mathrm{M}^{+}\right]$: 322.1600. Found: 265.0896 $\left[\mathrm{M}^{+}-t\right.$-butyl $]$.

(土)-5-[1-Tert-butyl-dimethyl-silanyloxy)-2-triisopropylsilanyloxymethyl-allyl]-benzo[1,3]dioxol (17)

To a solution of silyl ether $16(0.68 \mathrm{~g}, 2.12 \mathrm{mmol})$ in $15 \mathrm{~mL}$ of dry dichloromethane, under an atmosphere of argon, was added anhydrous triethylamine $(0.6 \mathrm{~mL}, 4.24$ $\mathrm{mmol})$, followed by triisopropylsilyl triflate $(0.74 \mathrm{~mL}$, $2.75 \mathrm{mmol})$. The resulting mixture was stirred for $1 \mathrm{~h}$ at room temperature. Then the reaction was diluted with dichloromethane $(25 \mathrm{~mL})$ and the organic phase was washed with a saturated solution of $(2 \times 50 \mathrm{~mL})$ and brine $(2 \times 50 \mathrm{~mL})$. The organic phase was then dried over anydrous magnesium sulfate and the solvent was removed under reduced pressure. The residue was purified by flash silica-gel column chromatography (eluent: hexane/ethyl acetate $2 \%$ ) to give 17 as colorless viscous oil in $92 \%$ yield. 
IR $v_{\max } / \mathrm{cm}^{-1}: 2948,2865,1650,1487,1246,1068,881$. ${ }^{1} \mathrm{H}$ NMR (500 MHz, $\mathrm{CDCl}_{3}$ ): $\delta-0.03$ (s, 3H), 0.06 (s, $3 \mathrm{H}), 0.86(\mathrm{~s}, 9 \mathrm{H}, t$-butyl), $1.05(\mathrm{~m}, 21 \mathrm{H}, 3 \mathrm{x}$ isopropyl), $4.0(\mathrm{~d}, J 14.2 \mathrm{~Hz}, 1 \mathrm{H}), 4.17(\mathrm{~d}, J 14.2 \mathrm{~Hz}, 1 \mathrm{H}), 5.19(\mathrm{~s}, 1 \mathrm{H}$, $\mathrm{CH}), 5.2$ (bs, 2H), $5.93\left(\mathrm{~d},{ }^{2} J 1.5 \mathrm{~Hz}, 2 \mathrm{H}\right), 6.72-6.85(3 \mathrm{H}$, $\mathrm{m}$, aromatics). ${ }^{13} \mathrm{C}$ NMR $\left(125 \mathrm{MHz}, \mathrm{CDCl}_{3}\right): \delta-5.0$; $-0.02 ; 11.9,18.0,18.3,25.8,62.6,75.5,100.8,106.8$, 107.6, 108.8, 119.5, 137.3, 146.5, 147.4, 151.0. MS (EI, 70eV) $m / z: 478\left(\mathrm{M}^{+}\right), 435$, 304, 265, 203, 173, 115, 73. HRMS (IE, 70eV) $m / z$ Calc. for $\mathrm{C}_{26} \mathrm{H}_{46} \mathrm{O}_{4} \mathrm{Si}_{2}[\mathrm{M}]^{+}$: 478.2935. Found: 478.2970.

(土)-3-Benzo[1,3]dioxol-5-yl-3-(tert-butyldimethylsilanyloxy)-2-triisopropyl-silanyloxymethyl-1-propanol (18a/18b)

To a solution of compound $17(0.20 \mathrm{~g}, 0.42 \mathrm{mmol})$ in $4 \mathrm{~mL}$ of dry tetrahydrofuran, at $0^{\circ} \mathrm{C}$, was added a solution of 9-BBN in THF $\left(0.5 \mathrm{~mol} \mathrm{~L}^{-1}\right)(6 \mathrm{~mL}, 3 \mathrm{mmol})$. The resulting mixture was stirred for $16 \mathrm{~h}$, at room temperature. After that, the mixture was cooled to $0{ }^{\circ} \mathrm{C}$ followed by the slow addition of $\mathrm{NaOH}$ solution $\left(5.0 \mathrm{~mL}, 3 \mathrm{~mol} \mathrm{~L}^{-1}\right)$ and $30 \% \mathrm{H}_{2} \mathrm{O}_{2}(5.0 \mathrm{~mL})$. The mixture was stirred for $45 \mathrm{~min}$, at room temperature. Then, a saturated solution of $\mathrm{NaHCO}_{3}$ $(5.0 \mathrm{~mL})$ was added. The final mixture was poured into a separatory funnel filled with dichloromethane $(50 \mathrm{~mL})$. The phases were separated, and the organic one was successively washed with a saturated solution of $\mathrm{NaHCO}_{3}$ $(10 \mathrm{~mL})$, distilled water $(10 \mathrm{~mL})$ and brine $(10 \mathrm{~mL})$. Finally, the organic phase was dried over anhydrous magnesium sulfate, and the solvent was removed under reduced pressure. The residue was purified by silica-gel column chromatography (using as eluting mixture hexane/ ethyl acetate $5 \%$ ) to give alcohols $\mathbf{1 8 a} / \mathbf{b}$ as a mixture of diastereoisomers in $75 \%$ yield.

IR $v_{\max } / \mathrm{cm}^{-1}: 3407,2927,2864,1487,1246,1059 .{ }^{1} \mathrm{H}$ NMR (300 MHz, $\mathrm{CDCl}_{3}$ ) Major diastereoisomer $( \pm)-\mathbf{1 8 a}$ : $\delta-0.17$ (s, 3H), 0.07 (s, 3H), 0.87 (s, 9H, $t$-butyl), 1.01$1.10(\mathrm{~m}, 21 \mathrm{H}, 3 \mathrm{x}$ isopropyl group), $1.78-1.88(\mathrm{~m}, 1 \mathrm{H}$, $\mathrm{CH}), 3.64\left(\mathrm{dd}, J 10 \mathrm{~Hz}, J 6.2 \mathrm{~Hz}, 1 \mathrm{H}_{a}, \mathrm{CH}_{2}\right), 3.68(\mathrm{dd}, J$ $\left.10 \mathrm{~Hz}, J 5.9 \mathrm{~Hz}, 1 \mathrm{H}_{b}, \mathrm{CH}_{2}\right), 3.76-3.90\left(\mathrm{~m}, 2 \mathrm{H}, \mathrm{CH}_{2}\right), 4.90$ $(\mathrm{d}, J 5.9 \mathrm{~Hz}, 1 \mathrm{H}, \mathrm{CH}), 5.92\left(\mathrm{~d}, J 1.5 \mathrm{~Hz}, 1 \mathrm{H}_{a}, \mathrm{CH}_{2}\right), 5.96$ (d, $\left.J 1.5 \mathrm{~Hz}, 1 \mathrm{H}_{b}, \mathrm{CH}_{2}\right), 6.74-6.84$ (m, $3 \mathrm{H}$ aromatics). Minor diastereoisomer $( \pm)-\mathbf{1 8 b}: \delta-0.19(\mathrm{~s}, 3 \mathrm{H}), 0.03$ (s, $3 \mathrm{H}), 0.87$ (s, 9H, $t$-butyl), 1.01-1.10 (m, $21 \mathrm{H}, 3 \times$ isopropyl group), 2.13-2.20 (m, 1H, CH), 3.57 (dd, $J 10.2 \mathrm{~Hz}, J 4.7$ $\mathrm{Hz}, 1 \mathrm{H}_{a}, \mathrm{CH}_{2}$ ), 3.6 (dd, J $10.2 \mathrm{~Hz}, J 4.0 \mathrm{~Hz}, 1 \mathrm{H}_{b}, \mathrm{CH}_{2}$ ), 3.76-3.90 (m, 2H, $\left.\mathrm{CH}_{2}\right), 4.72(\mathrm{~d}, J 6.6 \mathrm{~Hz}, 1 \mathrm{H}, \mathrm{CH}), 5.94$ $\left(\mathrm{d}, J 1.4 \mathrm{~Hz}, 1 \mathrm{H}_{a}, \mathrm{CH}_{2}\right), 5.95\left(\mathrm{~d}, J 1.4 \mathrm{~Hz}, 1 \mathrm{H}_{b}, \mathrm{CH}_{2}\right)$, 6.74-6.84 (m, 3H aromatics). ${ }^{13} \mathrm{C}$ NMR (75 MHz, $\mathrm{CDCl}_{3}$ ) Major diastereoisomer $( \pm)$-18a: $\delta-5.1,-4.5,12.018,18.1$, 25.3, 51, 63, 63.8, 74.8, 100.8, 106.7, 107.7, 119.6, 137.5,
146.5, 147.4. Minor diastereoisomer $( \pm)-18 b: \delta-5.1$, $-4.4,11.9,18,18.1,24.8,50.3,63.4,64.1,74.2,100.9$, 107, 107.7, 119.9, 136.3, 146.5, 147.4.

( \pm )-3-Benzo[1,3]dioxol-5-yl-3-(tert-butyl-dimethylsilanyloxy)-2-triisopropyl-silanyloxymethylpropionaldehyde (19)

To a mixture of diastereoisomeric alcohols $\mathbf{1 8 a} / \mathbf{b}$ $(0.17 \mathrm{~g}, 0.5 \mathrm{mmol}), \mathrm{N}$-methylmorpholine oxide (NMO) $(0.068 \mathrm{~g}, 0.75 \mathrm{mmol})$, ammonium tetrapropylperruthenate (TPAP) $(0.011 \mathrm{~g}, 10 \mathrm{mmol} \%, 0.03 \mathrm{mmol})$, molecular sieves $4 \AA(500 \mathrm{mg}$ per mmol of substrate, freshly activated and triturated) was added $4.0 \mathrm{~mL}$ of dry dichloromethane. The heterogeneous mixture was stirred, at room temperature, for $30 \mathrm{~min}$. After that, the mixture was filtered over a column filled with silica gel (230400 mesh), which was washed with dichloromethane. The filtrates were combined and the solvent was removed under reduced pressure to give aldehyde $19 \mathbf{a} / \mathbf{b}$ as a mixture of diastereoisomers in $85 \%$ yield. No additional purification was necessary and the product was used directly in the next step.

IR $v_{\max } / \mathrm{cm}^{-1}: 2943,2866,1726,1504,1488,1247 .{ }^{1} \mathrm{H}$ $\operatorname{NMR}\left(300 \mathrm{MHz}, \mathrm{CDCl}_{3}\right): \delta-0.17\left(\mathrm{~s}, 3 \mathrm{H}, \mathrm{CH}_{3}\right), 0.06$ (s, $3 \mathrm{H}, \mathrm{CH}_{3}$ ), 0.86 (s, 9H, t-butyl), 0.98-1.05 (m, 21H, $3 \times$ isopropyl), $2.55(\mathrm{~m}, 1 \mathrm{H}), 3.83(\mathrm{dd}, J 10.2 \mathrm{~Hz}, J 5.1 \mathrm{~Hz}$, 1H), 3.87 (dd, $J 10.2 \mathrm{~Hz}, J 5.1 \mathrm{~Hz}, 1 \mathrm{H}), 5.2$ (d, J $6.9 \mathrm{~Hz}$, $1 \mathrm{H}, \mathrm{CH}), 5.98\left(\mathrm{~s}, 2 \mathrm{H}, \mathrm{OCH}_{2} \mathrm{O}\right), 6.7-6.8(\mathrm{~m}, 3 \mathrm{H}$, aromatics), $9.8(\mathrm{~d}, J 2.9 \mathrm{~Hz}, 1 \mathrm{H}) .{ }^{13} \mathrm{C} \mathrm{NMR}\left(75 \mathrm{MHz}, \mathrm{CDCl}_{3}\right): \delta-5.1$, $-4.3,12,17.7,18,25.8,61,62.7,72.1,101,106.8,107.8$, 119.9, 136.4, 146.9, 147.6, 204. MS (EI, 70eV) $\mathrm{m} / \mathrm{z}: 494$ $\left(\mathrm{M}^{+}\right), 437,265,145,73$. HRMS (EI, 70eV) $\mathrm{m} / \mathrm{z}$ Calc. for $\mathrm{C}_{26} \mathrm{H}_{46} \mathrm{O}_{5} \mathrm{Si}_{2}\left[\mathrm{M}^{+}-t\right.$-butyl]: 437.2174. Found: 437.2249.

( \pm )-3-(Benzo[1,3]dioxol-5-yl-3-(tert-butyl-dimethylsilanyloxy)-2-triisopropylsilanyloxymethyl-propanoic acid (20)

To a mixture of aldehyde $\mathbf{1 9 a} / \mathbf{b}(0.26 \mathrm{~g}, 0.52 \mathrm{mmol})$, $4.3 \mathrm{~mL}$ of $t$-butanol and 2-methyl-but-2-ene (48.4 equiv.; $25.42 \mathrm{mmol}$ ), cooled to $0{ }^{\circ} \mathrm{C}$, was added a solution of $\mathrm{NaClO}_{2}(0.44 \mathrm{~g}, 9.2$ equiv., $2.42 \mathrm{mmol})$ and $\mathrm{NaH}_{2} \mathrm{PO}_{4}(0.44$ $\mathrm{g}, 6.9$ equiv., $1.82 \mathrm{mmol}$ ) in $1.5 \mathrm{~mL}$ of distilled water. The resulting heterogeneous mixture was stirred for $5 \mathrm{~h}$ at room temperature. Then the reaction mixture was concentrated under reduced pressure, the residue was diluted with distilled water $(30 \mathrm{~mL})$, which was acidified to $\mathrm{pH} 3$ (using a $10 \% \mathrm{HCl}$ solution). The aqueous phase was extracted with ethyl ether $(3 \times 20 \mathrm{~mL})$ and after separation, the organic layer was dried over anhydrous magnesium sulfate and the solvent was evaporated. The residue was purified by flash silica gel column chromatography (hexane/ethyl 
acetate $8 \%$ ) to provide the enriched acid 20a (syn) as a yellow viscous oil in $87 \%$ yield.

IR $v_{\max } / \mathrm{cm}^{-1}: 3448,2933,2864,1704,1490,1248 .{ }^{1} \mathrm{H}$ NMR (300 MHz, $\left.\mathrm{CDCl}_{3}\right): \delta-0.15$ (s, 3H), 0.08 (s, 3H), 0.87 (s, 9H, $t$-butyl), $-1.0-1.09$ (m, $21 \mathrm{H}, 3 \times$ isopropyl), $2.86(\mathrm{~m}, 1 \mathrm{H}), 3.73(\mathrm{dd}, J 10.2 \mathrm{~Hz}, J 5.1 \mathrm{~Hz}, 1 \mathrm{H}), 3.75(\mathrm{dd}$, $J 10.2 \mathrm{~Hz}, J 5.1 \mathrm{~Hz}, 1 \mathrm{H}), 5.0(\mathrm{~d}, J 6.6 \mathrm{~Hz}, 1 \mathrm{H}), 5.96(\mathrm{~d}, J$ $\left.1.5 \mathrm{~Hz}, \mathrm{OCH}_{2} \mathrm{O}\right), 6.75-6.84$ (m, 3H, aromatics.). ${ }^{13} \mathrm{C}$ NMR $\left(75 \mathrm{MHz}, \mathrm{CDCl}_{3}\right): \delta-5.3,-4.5,11.9,17.6,18,25.7,57.3$, 61.6, 72.7, 101, 106.7, 107.9, 119.9, 135.6, 147.1, 147,6, 173.9. HRMS (EI, 70 eV) $m / z$ Calc. for $\mathrm{C}_{26} \mathrm{H}_{46} \mathrm{O}_{6} \mathrm{Si}_{2}\left[\mathrm{M}^{+}-\right.$ t-butyl]: 453.2123 . Found: 453.2279.

(土)-[2-Benzo[1,3]dioxol-5-yl-2-(tert-butyl-dimethylsilanyloxy)-1-triisopropylsilanyloxymethyl-ethyl]methoxycarboxamide (24)

To a solution of acid $20 \mathrm{a}(0.1 \mathrm{~g}, 0.195 \mathrm{mmol})$ in acetone $(1.9 \mathrm{~mL})$ cooled at $0{ }^{\circ} \mathrm{C}$ was added anhydrous triethylamine $(54 \mathrm{~mL}, 0.39 \mathrm{mmol})$ and ethyl chloroformate $(28 \mathrm{~mL}, 0.29$ mmol). The mixture was stirred for $45 \mathrm{~min}$ at $0{ }^{\circ} \mathrm{C}$. After this time, the formation of an acyl carbonate was observed by tlc $\left(\mathrm{R}_{\mathrm{f}}=0.6\right.$; hexane/ethyl acetate $\left.20 \%\right)$. To this mixture was then added $57 \mathrm{~mL}$ of a solution of sodium azide $(0.019 \mathrm{~g}, 0.29 \mathrm{mmol})$ in distilled water $(3 \mathrm{~mL})$. The mixture was stirred for $2 \mathrm{~h}$, at room temperature. After this time, an acylazide was formed $\left(\mathrm{R}_{\mathrm{f}}=0.62\right.$, hexane/ ethyl acetate $20 \%$ ). Then the mixture was diluted with dichloromethane $(20 \mathrm{~mL})$ and the organic phase was washed with distilled water $(5 \mathrm{~mL})$ and brine $(5 \mathrm{~mL})$, dried over sodium sulfate and finally the solvent was removed under reduced presurre. The residue was dissolved in anhydrous toluene $(10 \mathrm{~mL})$ and refluxed for $2 \mathrm{~h}$, under an argon atmosphere. The reaction was followed by infrared spectroscopy. At the end of reaction the absorption band at $2136 \mathrm{~cm}^{-1}$ (atributted to acylazide) had disappeared and a new absorption band at $2254 \mathrm{~cm}^{-1}$ confirmed the formation of an isocyanate. Toluene was evaporated and the residue was dissolved in anhydrous methanol $(5 \mathrm{~mL})$. The resulting mixture was refluxed for $12 \mathrm{~h}$. Then, the solvent was removed under reduced pressure and the residue was purified by flash silica gel column chromatography (hexane/ethyl acetate $15 \%$ ) to provide the carbamate $\mathbf{2 4}$ as a viscous colorless oil in an overall yield of $65 \%$ (for 4 steps).

IR $v_{\max } / \mathrm{cm}^{-1}: 3450,2947,2866,1731,1251,1101$, 776. ${ }^{1} \mathrm{H}$ NMR (300 MHz, $\left.\mathrm{CDCl}_{3}\right): \delta-0.11(\mathrm{~s}, 3 \mathrm{H}), 0.07$ (s, $3 \mathrm{H}), 0.85(\mathrm{~s}, 9 \mathrm{H}, t$-butyl $), 0.91-1.07(\mathrm{~m}, 21 \mathrm{H}, 3 \times$ isopropyl), $3.58(\mathrm{bs}, 3 \mathrm{H}), 3.60-3.72(\mathrm{~m}, 3 \mathrm{H}), 5.02(\mathrm{~m}, 2 \mathrm{H}$, benzylic $\mathrm{CH}$ and $\mathrm{NH}), 5.95\left(\mathrm{~s}, 2 \mathrm{H}, \mathrm{OCH}_{2} \mathrm{O}\right), 6.74-6.8(\mathrm{~m}$, $3 \mathrm{H}$, aromatics) $.^{13} \mathrm{C} \mathrm{NMR}\left(75 \mathrm{MHz}, \mathrm{CDCl}_{3}\right): \delta-5.1,-4.5$, 12.1, 17.8, 18.1, 25.9, 52, 59.2, 62, 71.4, 100.8, 106.7,
107.8, 119.3, 136.5, 146.6, 147.3, 156.5. MS (EI, 70eV) $m / z: 496\left(\mathrm{M}^{+}\right), 364,267,265,135,73$. HRMS (EI, 70eV) $m / z$ Calc. for $\mathrm{C}_{27} \mathrm{H}_{49} \mathrm{NO}_{6} \mathrm{Si}_{2}\left[\mathrm{M}^{+}-t\right.$-butyl]: 482.2389 . Found: 482.2491 .

\section{( \pm -Methyl 2-\{1,3-benzodioxol-5-yl[(4-methoxybenzyl) oxy]methyl;acrylate (25)}

To a solution of adduct $\mathbf{1 3}(0.47 \mathrm{~g}, 2 \mathrm{mmol})$ in dry dichloromethane $(4.5 \mathrm{~mL})$ was added $p$-methoxybenzyl trichloroacetimidate $(0.85 \mathrm{~g}, 3 \mathrm{mmol})$ and a catalytic amount of camphorsulfonic acid (0.023 g, $0.1 \mathrm{mmol})$. The resulting solution was stirred for $18 \mathrm{~h}$ at room temperature. Then, the reaction mixture was diluted with diethyl ether $(25 \mathrm{~mL})$ and the organic phase was washed with a saturated solution of $\mathrm{NaHCO}_{3}(2 \times 10 \mathrm{~mL})$, distilled water $(2 \times 10 \mathrm{~mL})$ and brine $(2 \times 10 \mathrm{~mL})$. The organic phase was then dried over sodium sulfate and the solvent was evaporated. The residue was purified by flash silica gel colum chromatography (hexane/ethyl acetate 5\%) to afford PMB ether $\mathbf{2 5}$ as a colorless viscous oil in $93 \%$ yield.

IR $v_{\max } / \mathrm{cm}^{-1}: 2952,2901,2840,1721,1612,1513$, 1486, 1441, 1247, 1068. ${ }^{1} \mathrm{H}$ NMR (500 MHz, $\left.\mathrm{CDCl}_{3}\right)$ : $\delta 3.68$ (s, 3H), 3.80 (s, 3H), 4.39 (dd, $J 19$ and $11.5 \mathrm{~Hz}$, $2 \mathrm{H}), 5.22(\mathrm{~s}, 1 \mathrm{H}), 5.94\left(\mathrm{~s}, 2 \mathrm{H}, \mathrm{CH}_{2} \mathrm{OCH}_{2}\right), 6.01(\mathrm{~s}, 1 \mathrm{H})$, $6.32(\mathrm{~s}, 1 \mathrm{H}), 6.75-6.98(\mathrm{~m}, 5 \mathrm{H}$ aromatics), $7.26(\mathrm{~d}, J 8.8$ $\mathrm{Hz}, 2 \mathrm{H}$ aromatics). ${ }^{13} \mathrm{C} \mathrm{NMR}\left(125 \mathrm{MHz}, \mathrm{CDCl}_{3}\right): \delta 51.7$, 55.2, 70.2, 77.8, 101, 107.9, 108, 113.7, 121.6, 124.9, $129.3,130.1,133.5,141.2,147.2,147.7,159.1,166.2$. MS (EI, 70eV) m/z: $356\left(\mathrm{M}^{+}\right), 220,160,149,137,121$, 102, 77. HRMS (EI, 70eV) $\mathrm{m} / z$ Calc. for $\mathrm{C}_{20} \mathrm{H}_{20} \mathrm{O}_{6}\left[\mathrm{M}^{+}\right]$: 356.1260. Found: 356.1262 .

\section{(土)-2-\{1,3-Benzodioxol-5-yl[(4-methoxybenzyl) oxy]methyl?prop-2-en-1-ol (26)}

A solution of ether $25(0.35 \mathrm{~g}, 1 \mathrm{mmol})$ in dry dichloromethane $(7 \mathrm{~mL})$ was cooled to $-78{ }^{\circ} \mathrm{C}$, under an atmosphere of argon. Then a solution of DIBAL-H in toluene $\left(1.0 \mathrm{~mol} \mathrm{~L}^{-1}\right)(2.4 \mathrm{~mL}, 2.4 \mathrm{mmol})$ was slowly added. The resulting solution was stirred for $2 \mathrm{~h}$, at $-78^{\circ} \mathrm{C}$. After that a saturated solution of sodium acetate was added ( 2.2 $\mathrm{mL}$ ) and the reaction was allowed to warm to the room temperature. The reaction medium was then poured into a beaker filled with ethyl ether $(50 \mathrm{~mL})$ and a saturated solution of $\mathrm{NH}_{4} \mathrm{Cl}(4.6 \mathrm{~mL})$. The mixture was stirred for $1 \mathrm{~h}$ until we observed the formation of a gel, which was then filtered over a pad of Celite ${ }^{\circledR}$. The solid was washed with small portions of diethyl ether and the organic phases were combined, dried over magnesium sulfate and finally the solvent was removed under reduced pressure. The residue was purified by flash silica gel column chroma- 
tography (hexane/ ethyl acetate 5\%) to furnish $\mathbf{2 6}$ as a colorless fluid oil in $72 \%$ yield.

IR $v_{\max } / \mathrm{cm}^{-1}: 3417,2897,2840,1612,1513,1441$, 1247, 1038. ${ }^{1} \mathrm{H}$ NMR (300 MHz, $\mathrm{CDCl}_{3}$ ): $\delta 2.04$ (bs, $1 \mathrm{H}$, $\mathrm{OH}), 3.80(\mathrm{~s}, 3 \mathrm{H}), 4.01\left(\mathrm{~d},{ }^{2} J 12.8 \mathrm{~Hz}, 1 \mathrm{H}\right), 4.11\left(\mathrm{~d},{ }^{2} J\right.$ $12.8 \mathrm{~Hz}, 1 \mathrm{H}), 4.38\left(\mathrm{~d},{ }^{2} J 11.5 \mathrm{~Hz}, 1 \mathrm{H}\right), 4.45\left(\mathrm{~d},{ }^{2} J 11.5\right.$ $\mathrm{Hz}, 1 \mathrm{H}), 4.88(\mathrm{~s}, 1 \mathrm{H}$, benzylic $\mathrm{CH}), 5.11(\mathrm{~s}, 1 \mathrm{H}), 5.24(\mathrm{~s}$, $1 \mathrm{H}), 5.96\left(\mathrm{~s}, 2 \mathrm{H}, \mathrm{OCH}_{2} \mathrm{O}\right), 6.78-6.97(\mathrm{~m}, 5 \mathrm{H}$ aromatics), 7.22 (d, $J 8.0 \mathrm{~Hz}, 2 \mathrm{H}$ aromatics). ${ }^{13} \mathrm{C}$ NMR $(75 \mathrm{MHz}$, $\left.\mathrm{CDCl}_{3}\right): \delta 55.2,63.7,70,81.9,101,107.4,108,113.7$, $113.8,120.6,129.4,130,133.7,147.1,147.9,148.3$, 159.2. HRMS (EI, $70 \mathrm{eV}) \mathrm{m} / \mathrm{z}$ Calc. for $\mathrm{C}_{19} \mathrm{H}_{20} \mathrm{O}_{5}\left[\mathrm{M}^{+}\right]$: 328.1311. Found: 328.1289 .

\section{5-[1-[(4-Methoxybenzyl)oxy]-2-(tert-butyl-diphenyl} silyloxy)prop-2-enyl]-1,3-benzodioxole (27)

To a solution of $26(0.328 \mathrm{~g}, 1 \mathrm{mmol})$ in dichloromethane $(6.0 \mathrm{~mL})$, under an atmosphere of argon, was added $N, N$-dimethylaminopyridine (DMAP) $(0.003 \mathrm{~g}$, $0.024 \mathrm{mmol})$, dry triethylamine $(0.20 \mathrm{~mL}, 1.5 \mathrm{mmol})$ and tert-butyldiphenylsilyl chloride $(0.4 \mathrm{~mL}, 1.5 \mathrm{mmol})$. The resulting mixture was stirred for $16 \mathrm{~h}$, at room temperature. After that the mixture was diluted with dichloromethane $(25 \mathrm{~mL})$ and the organic phase was washed with a saturated solution of $\mathrm{NaHCO}_{3}(2 \times 20 \mathrm{~mL})$. The phases were separated. The organic layer was dried over magnesium sulfate and the solvent was evaporated. The residue was purified by silica gel column chromatography (hexane/ ethyl acetate $2 \%$ ) to provide $\mathbf{2 7}$ as colorless viscous oil in an almost quantitative yield.

IR $v_{\max } / \mathrm{cm}^{-1}: 3071,2956,2857,1612,1513,1486$, 1246, 1073. ${ }^{1} \mathrm{H}$ NMR (300 MHz, $\left.\mathrm{CDCl}_{3}\right): \delta 1.03(\mathrm{~s}, 9 \mathrm{H})$, $3.78(\mathrm{~s}, 3 \mathrm{H}), 4.05\left(\mathrm{~d},{ }^{2} J 14.2 \mathrm{~Hz}, 1 \mathrm{H}\right), 4.13\left(\mathrm{~d},{ }^{2} J 14.2 \mathrm{~Hz}\right.$, $1 \mathrm{H}), 4.35\left(\mathrm{~s}, 2 \mathrm{H}, \mathrm{CH}_{2}\right), 4.80(\mathrm{~s}, 1 \mathrm{H}, \mathrm{CH}), 5.25\left(\mathrm{~s}, 1 \mathrm{H}_{b}\right.$, $\mathrm{CH}_{2}$ ), $5.43\left(\mathrm{~s}, 1 \mathrm{H}_{a}, \mathrm{CH}_{2}\right), 5.92\left(\mathrm{~s}, 2 \mathrm{H}, \mathrm{CH}_{2}\right), 6.7-7.64(\mathrm{~m}$, $17 \mathrm{H}$, aromatics). ${ }^{13} \mathrm{C}$ NMR $\left(75 \mathrm{MHz}, \mathrm{CDCl}_{3}\right): \delta 19.2,26.8$, 55.2, 63.7, 69.8, 80.5, 100.9, 107.4, 107.9, 111.5, 113.7, $120.6,127.6,129.2,129.6,130.4,133.5,134.1,135.5$, 146.9, 147.6, 147.9, 159.1. MS (EI, 70eV) $\mathrm{m} / z: 566\left(\mathrm{M}^{+}\right)$, $200,199,121$. HRMS (EI, 70eV) $m / z$ Calc. for $\mathrm{C}_{35} \mathrm{H}_{38} \mathrm{O}_{5} \mathrm{Si}$ $[\mathrm{M}]^{+}:$566.2489. Found: 566.2475.

\section{3-(1,3-Benzodioxol-5-yl)-3-[(4-methoxybenzyl)oxy]-2-} (tert-butyl-diphenylsilyloxy)propan-1-ol (28)

A solution of $27(0.2 \mathrm{~g}, 0.35 \mathrm{mmol})$ in anhydrous THF $(3.0 \mathrm{~mL})$ was cooled at $0{ }^{\circ} \mathrm{C}$ and then a solution of 9-BBN $\left(0.5 \mathrm{~mol} \mathrm{~L}^{-1}\right)$ in THF $(5.0 \mathrm{~mL}, 2.5 \mathrm{mmol})$ was added drop by drop under an argon atmosphere. After finishing the addition, the solution was stirred for $16 \mathrm{~h}$ at room temperature. After that time, the reaction mixture was cooled to $0{ }^{\circ} \mathrm{C}$ and then a solution $3 \mathrm{~mol} \mathrm{~L}^{-1}$ of $\mathrm{NaOH}(3.6$
$\mathrm{mL}$ ) was slowly added, followed by $30 \% \mathrm{H}_{2} \mathrm{O}_{2}(3.6 \mathrm{~mL})$. The resulting mixture was stirred at $0{ }^{\circ} \mathrm{C}$ for $45 \mathrm{~min}$. After that, a saturated solution of $\mathrm{NaHCO}_{3}$ was added $(5.0 \mathrm{~mL})$ and the resulting mixture was transferred to a separatory funnel with dichloromethane $(50 \mathrm{~mL})$. The phases were separated and the organic one was washed with a saturated solution of $\mathrm{NaHCO}_{3}(2 \times 10 \mathrm{~mL})$, distilled water $(2 \times 10$ $\mathrm{mL})$ and brine $(2 \times 10 \mathrm{~mL})$. The organic phase was dried over anhydrous sodium sulfate and the solvent was evaporated. The residue was purified by flash silica gel column chromatography (hexane/ethyl acetate $5 \%$ ) to afford alcohols $\mathbf{2 8 a} / \mathbf{b}$, as a mixture of diastereoisomers, in $67 \%$ yield.

IR $v_{\max } / \mathrm{cm}^{-1}: 3433,2930,2856,1486,1245,1112$, 1037, 702. ${ }^{1} \mathrm{H}$ NMR (300 MHz, $\mathrm{CDCl}_{3}$ ) major diastereoisomer $( \pm)$-28a: $\delta 1.03(\mathrm{~s}, 9 \mathrm{H}), 1.96(\mathrm{~m}, 1 \mathrm{H}, \mathrm{CH}), 3.43$ (dd, $\left.{ }^{2} J 10.3 \mathrm{~Hz},{ }^{3} J 5.3 \mathrm{~Hz}, 1 \mathrm{H}\right), 3.57\left(\mathrm{dd},{ }^{2} J 10.4 \mathrm{~Hz},{ }^{3} J 4.2\right.$ $\mathrm{Hz}, 1 \mathrm{H}), 3.81\left(\mathrm{~s}, 3 \mathrm{H}, \mathrm{CH}_{3}\right), 3.85-4.1\left(\mathrm{~m}, 2 \mathrm{H}, \mathrm{CH}_{2}\right), 4.14$ $\left(\mathrm{d},{ }^{2} J 11 \mathrm{~Hz}, 1 \mathrm{H}_{a}, \mathrm{CH}_{2}\right), 4.4\left(\mathrm{~d},{ }^{2} J 11 \mathrm{~Hz}, 1 \mathrm{H}_{b}, \mathrm{CH}_{2}\right), 4.53$ (d, ${ }^{3}$ J $\left.8.4 \mathrm{~Hz}, 1 \mathrm{H}, \mathrm{CH}\right), 5.99$ (s, 2H), 6.79-6.82 (m, 4H aromatics), 6.84 (bs, $1 \mathrm{H}$ aromatic), $7.06(\mathrm{~d}, J 8.7 \mathrm{~Hz}, 1 \mathrm{H}$ aromatic), 7.32-7.72 (m, 10H aromatics). Minor diastereoisomer ( \pm )-28b: d $1.07\left(\mathrm{~s}, 9 \mathrm{H}, 3 \mathrm{CH}_{3}\right), 2.08(\mathrm{~m}$, $1 \mathrm{H}, \mathrm{CH}), 3.5\left(\mathrm{dd},{ }^{2} J 11.4 \mathrm{~Hz},{ }^{3} \mathrm{~J} 5.5 \mathrm{~Hz}, 1 \mathrm{H}\right), 3.64\left(\mathrm{dd},{ }^{2} J\right.$ $\left.11.4 \mathrm{~Hz},{ }^{3} \mathrm{~J} 4.2 \mathrm{~Hz}, 1 \mathrm{H}\right), 3.80$ (s, 3H), 3.85-4.1 (m, $\left.2 \mathrm{H}\right)$, $4.11\left(\mathrm{~d},{ }^{2} J 11.3 \mathrm{~Hz}, 1 \mathrm{H}\right), 4.33\left(\mathrm{~d},{ }^{2} J 11.3 \mathrm{~Hz}, 1 \mathrm{H}\right), 4.49$ (d, $\left.{ }^{3} J 8.4 \mathrm{~Hz}, 1 \mathrm{H}\right), 5.99$ (s, 2H), 6.79-6.82 (m, $4 \mathrm{H}$ aromatics), 6.84 (bs, $1 \mathrm{H}$ aromatic), 7.06 (d, $J 8.7 \mathrm{~Hz}, 1 \mathrm{H}$ aromatic), 7.32-7.72 (m, $10 \mathrm{H}$ aromatics). ${ }^{13} \mathrm{C} \mathrm{NMR} \mathrm{(75} \mathrm{MHz,} \mathrm{CDCl}_{3}$ ) Major diastereoisomer ( \pm )-28a: d 19.2, 26.9, 49.7, 55.6, 63.1, 63.9, 70.2, 81.5, 101, 107.3, 107.9, 113.9, 121.1, $127.7,129.6,129.8,129.9,133.1,134.2,135.6,147.2$, 148, 159.3. Minor diastereoisomer $( \pm)-28 b$ : d 19.2, 26.8, 48.8, 55.3, 62.8, 63.7, 70.1, 79.2, 101, 107.4, 108, 113.7, 121.1, 127.6, 129.3, 129.7, 130.3, 133.2, 133.9, 135.6, 147.2, 148, 159.1. HRMS (EI, 70eV) $\mathrm{m} / \mathrm{z}$ Calc. for $\mathrm{C}_{35} \mathrm{H}_{40} \mathrm{O}_{6} \mathrm{Si}\left[\mathrm{M}^{+}\right]$: 584.2594. Found: 584.2620.

(土)-3-(1,3-Benzodioxol-5-yl)-3-[(4-methoxybenzyl)oxy]-2(tert-butyl-diphenylsilyloxy)propanal (29)

To a mixture of diastereoisomeric alcohols $28 \mathrm{a} / \mathbf{b}(0.2 \mathrm{~g}$, $0.34 \mathrm{mmol}), \mathrm{N}$-methyl morpholine oxide (NMO) (0.068 g, $0.5 \mathrm{mmol}$ ), ammonium tetrapropylperruthenate (TPAP) $(0.011 \mathrm{~g}, 10 \mathrm{mmol} \%, 0.03 \mathrm{mmol})$ and triturated and activated $4 \AA$ molecular sieves $(0.16 \mathrm{~g}, 0.5 \mathrm{~g}$ per mmol of substrate) was added anydrous dichloromethane $(4.0 \mathrm{~mL})$. The resulting heterogenous mixture was stirred for $1 \mathrm{~h}$ at room temperature. After that time, the mixture was filtered on a column containing flash silica gel (approximately $5.0 \mathrm{~cm}$ of silica gel) and the solid was washed with $\mathrm{CH}_{2} \mathrm{Cl}_{2}$. The organic phases were combined and the solvent 
evaporated to obtain $\mathbf{2 9 a} / \mathbf{b}$ as colorless oil in $80 \%$ yield. No additional purification was necessary and the product was used in next step without any purification.

IR $v_{\max } / \mathrm{cm}^{-1}: 2928,2859,1725,1604,1499,1247$, 1104. ${ }^{1} \mathrm{H}$ NMR (500 MHz, $\mathrm{CDCl}_{3}$ ) Major diastereoisomer ( \pm )-29a: $\delta 1.01\left(\mathrm{~s}, 9 \mathrm{H}, 3 \mathrm{CH}_{3}\right), 2.64(\mathrm{~m}, 1 \mathrm{H}, \mathrm{CH}), 3.82(\mathrm{~s}$, $\left.3 \mathrm{H}, \mathrm{CH}_{3}\right), 3.76\left(\mathrm{dd},{ }^{2} J 10.5 \mathrm{~Hz},{ }^{3} J 3.8 \mathrm{~Hz}, 1 \mathrm{H}_{a}, \mathrm{CH}_{2}\right), 3.95$ $\left(\mathrm{dd},{ }^{2} J 10.5 \mathrm{~Hz},{ }^{3} J 4.5 \mathrm{~Hz}, 1 \mathrm{H}_{b}, \mathrm{CH}_{2}\right), 4.18\left(\mathrm{~d},{ }^{2} J 11.3 \mathrm{~Hz}\right.$, $\left.1 \mathrm{H}_{a}, \mathrm{CH}_{2}\right), 4.44\left(\mathrm{~d},{ }^{2} \mathrm{~J} 11.3 \mathrm{~Hz}, 1 \mathrm{H}_{b}, \mathrm{CH}_{2}\right), 4.88$ (d, ${ }^{3} J 9.1$ $\mathrm{Hz}, 1 \mathrm{H}, \mathrm{CH}), 5.99$ (bs, 2H), 6.62-6.85 (m, 4H aromatics), 7.16-7.68 (m, 13H aromatics), $9.87\left(\mathrm{~d},{ }^{3} J 3.0 \mathrm{~Hz}, 1 \mathrm{H}\right)$. Minor diastereoisomer $( \pm)-29 b: \delta 1.05\left(\mathrm{~s}, 9 \mathrm{H}, 3 \mathrm{CH}_{3}\right), 2.88$ (m, 1H, CH), 3.81 (s, 3H, $\left.\mathrm{CH}_{3}\right), 3.57$ (dd, ${ }^{2} J 10.4 \mathrm{~Hz},{ }^{3} J$ $\left.4.9 \mathrm{~Hz}, 1 \mathrm{H}_{a}, \mathrm{CH}_{2}\right), 3.87\left(\mathrm{dd},{ }^{2} J 10.4 \mathrm{~Hz},{ }^{3} J 4.9 \mathrm{~Hz}, 1 \mathrm{H}_{b}\right.$, $\left.\mathrm{CH}_{2}\right), 4.22\left(\mathrm{~d},{ }^{2} \mathrm{~J} 11.3 \mathrm{~Hz}, 1 \mathrm{H}_{a}, \mathrm{CH}_{2}\right), 4.40\left(\mathrm{~d},{ }^{2} J 11.3 \mathrm{~Hz}\right.$, $\left.1 \mathrm{H}_{b}, \mathrm{CH}_{2}\right), 4.82\left(\mathrm{~d},{ }^{3} \mathrm{~J} 7.6 \mathrm{~Hz}, 1 \mathrm{H}, \mathrm{CH},\right), 5.98$ (s, 2H), 6.62-6.85 (m, 4H aromatics), 7.16-7.68 (m, $13 \mathrm{H}$ aromatics), 9.73 (d, $\left.{ }^{3} \mathrm{~J} 2.4 \mathrm{~Hz}, 1 \mathrm{H}\right) .{ }^{13} \mathrm{C} \mathrm{NMR}(125 \mathrm{MHz}$, $\mathrm{CDCl}_{3}$ ) Major diastereoisomer ( \pm )-29a: d 19.1, 26.7, 55.2, 60.3, 61.2, 69.9, 77.1, 101.1, 107.3, 108, 113.8, 121.4, $127.7,129.3,129.7,129.8,132.6,132.8,135.5,147.5$, 148.1, 159.2, 203.7. Minor diastereoisomer $( \pm)-29 b$ : $\delta$ 19.2, 26.7, 55.2, 60.3, 60.4, 70, 77.2, 101.1, 107.3, 108.2, 113.7, 121, 127.6, 129.6, 129.7, 129.8, 132.5, 132.9, 135.4, 147.4, 148.1, 159.1, 202.7. MS (EI, 70eV) m/z: $582\left(\mathrm{M}^{+}\right)$, 199, 149, 135, 121, 77, 57. HRMS (EI, 70eV) m/z Calc. for $\mathrm{C}_{35} \mathrm{H}_{38} \mathrm{O}_{6} \mathrm{Si}\left[\mathrm{M}^{+}\right]$: 582.2438. Found: 582.2489.

\section{(土)-3-(1,3-Benzodioxol-5-yl)-3-[(4-methoxybenzyl)oxy]-2-} (tert-butyl-diphenylsilyloxymethyl)propanoic acid (30)

A mixture of diastereoisomeric aldehydes $( \pm)-29 a / 29 b$ $(0.11 \mathrm{~g}, 0.18 \mathrm{mmol}), t$-butanol $(3.85 \mathrm{~mL})$ and 2-methylbut-2-en $(0.95 \mathrm{~mL}, 8.85 \mathrm{mmol})$ was cooled to $0{ }^{\circ} \mathrm{C}$, then a solution of $\mathrm{NaClO}_{2}(0.15 \mathrm{~g}, 1.68 \mathrm{mmol})$ and $\mathrm{NaH}_{2} \mathrm{PO}_{4}$ $(0.15 \mathrm{~g}, 1.26 \mathrm{mmol})$ dissolved in $1 \mathrm{~mL}$ of distilled water was slowly added. The resulting mixture was stirred for $1 \mathrm{~h}$ at room temperature. After that, the volative components were evaporated and the residue was dissolved in distilled water $(20 \mathrm{~mL})$, acidified to $\mathrm{pH} 3$ with a $10 \%$ solution of $\mathrm{HCl}$. The aqueous phase was extracted with ethyl ether. The organic phases were combined, dried over anhydrous magnesium sulfate and the solvent was evaporated. The residue was purified by flash silica gel column chromatography (hexane/ethyl acetate $8 \%$ ) to give $\mathbf{3 0}$ as a mixture of diastereoisomers in $88 \%$ yield.

IR $v_{\max } / \mathrm{cm}^{-1}: 3058,2937,2891,1711,1609,1487$, 1245, 1105, 815, 700. ${ }^{1} \mathrm{H}$ NMR (500 MHz, $\mathrm{CDCl}_{3}$ ) Major diastereoisomer $( \pm)$-30a: $\delta 1.02\left(\mathrm{~s}, 9 \mathrm{H}, 3 \mathrm{CH}_{3}\right), 3.04-3.08$ $(\mathrm{m}, 1 \mathrm{H}, \mathrm{CH}), 3.8\left(\mathrm{~s}, 3 \mathrm{H}, \mathrm{CH}_{3}\right), 3.5-4.02\left(\mathrm{~m}, 2 \mathrm{H}, \mathrm{CH}_{2}\right)$, $4.13\left(\mathrm{~d},{ }^{2} \mathrm{~J} 11.3 \mathrm{~Hz}, 1 \mathrm{H}\right), 4.34$ (d, $\left.{ }^{2} J 11.3 \mathrm{~Hz}, 1 \mathrm{H}\right), 4.62$ (d, $\left.{ }^{3} J 9.4 \mathrm{~Hz}, 1 \mathrm{H}, \mathrm{CH}\right), 5.98\left(\mathrm{~s}, 2 \mathrm{H}, \mathrm{CH}_{2}\right), 6.6-7.7(\mathrm{~m}, 17 \mathrm{H}$, aromatics). Minor diastereoisomer $( \pm)-\mathbf{3 0 b}: \delta 0.98(\mathrm{~s}, 9 \mathrm{H}$, $\left.3 \mathrm{CH}_{3}\right), 2.9-2.95(\mathrm{~m}, 1 \mathrm{H}, \mathrm{CH}), 3.77$ (s, 3H, $\left.\mathrm{CH}_{3}\right), 3.5-4.02$ $\left(\mathrm{m}, 2 \mathrm{H}, \mathrm{CH}_{2}\right), 4.18\left(\mathrm{~d},{ }^{2} \mathrm{~J} 11.3 \mathrm{~Hz}, 1 \mathrm{H}_{a}, \mathrm{CH}_{2}\right), 4.37\left(\mathrm{~d},{ }^{2} \mathrm{~J}\right.$ $\left.11.3 \mathrm{~Hz}, 1 \mathrm{H}_{b}, \mathrm{CH}_{2}\right), 4.56\left(\mathrm{~d},{ }^{3} J 8.5 \mathrm{~Hz}, 1 \mathrm{H}, \mathrm{CH}\right), 5.92$ (s, $\left.2 \mathrm{H}, \mathrm{CH}_{2}\right), 6.6-7.7\left(\mathrm{~m}, 17 \mathrm{H}\right.$, aromatics). ${ }^{13} \mathrm{C}$ NMR $(125$ $\mathrm{MHz}, \mathrm{CDCl}_{3}$ ) Major diastereoisomer ( \pm )-30a: d 19.1, 26.7, 55.1, 62.3, 70, 77.9, 101, 107.2, 108, 113.7, 121.4, 127.7, $129.5,129.6,132.3,132.5,133.1,135.5,147.5,147.9$, 159.1, 177.4. Minor diastereoisomer $( \pm)-30 b: \mathrm{d} 19.1,26.6$, 55.2, 55.5, 62.1, 69.9, 78.4, 101.1, 107.4, 108, 113.7, 121.5, 127.6, 129.6, 132.4, 132.7, 134, 135.4, 147.6, 148, 159.2, 176.1. HRMS (EI, $70 \mathrm{eV}$ ) Calc. for $\mathrm{C}_{35} \mathrm{H}_{38} \mathrm{O}_{6} \mathrm{Si}$ $\left[\mathrm{M}^{+}\right.$- $t$-butyl] 524.16552. Found: 524.16533.

[2-Benzo[1,3]dioxol-5-yl)-1-(tert-butyl-diphenylsilanyloxymethyl)-2-(4-methoxy-benzyloxy)-ethyl]methoxycarboxamide, ( \pm )-31a/b

To a solution of diastereoisomeric acids $\mathbf{3 0 a} / \mathbf{b}(0.1 \mathrm{~g}$, $0.167 \mathrm{mmol})$ in acetone $(1.6 \mathrm{~mL})$, at $0{ }^{\circ} \mathrm{C}$, was added anhydrous triethylamine $(24 \mu \mathrm{L}, 0.33 \mathrm{mmol})$ and ethyl chloroformate $(24 \mu \mathrm{L}, 0.25 \mathrm{mmol})$. The mixture was then stirred for $45 \mathrm{~min}$ at $0{ }^{\circ} \mathrm{C}$. After observing the formation of an acyl carbonate (by TLC), a solution of sodium azide $(49 \mu \mathrm{L}, 0.0163 \mathrm{~g}, 0.25 \mathrm{mmol})$ in distilled water $(3 \mathrm{~mL})$ was added. The resulting mixture was stirred for $2 \mathrm{~h}$, until the formation of an acyl azide (observed by IR spectroscopy). Then, the reaction was diluted with dichloromethane and the organic phase was washed with distilled water $(5 \mathrm{~mL})$ and brine $(5 \mathrm{~mL})$, dried over anydrous sodium sulfate and finally the solvent was evaporated. To the residue was added dry toluene (10 $\mathrm{mL}$ ) and the solution was refluxed for $2 \mathrm{~h}$, under an argon atmosphere. The reaction was followed by IR spectroscopy until the appearance of an absorption band at 2259 $\mathrm{cm}^{-1}$, atributted to the isocyanate formed after the Curtius rearrangement. Then, the solvent was evaporated and to the residue was added anhydrous methanol. The solution was refluxed for $12 \mathrm{~h}$. After solvent evaporation, the residue was purified by flash silica gel column chromatography (hexane/ethyl acetate $15 \%$ ) to afford carbamate $\mathbf{3 1 a} / \mathbf{b}$ as a viscous colorless oil in an overall yield of $60 \%$ (four steps).

IR $v_{\max } / \mathrm{cm}^{-1}: 3436,2928,2857,1725,1612,1513$, 1247, 1112, 707. ${ }^{1} \mathrm{H}$ NMR (300 $\left.\mathrm{MHz}, \mathrm{CDCl}_{3}\right) \delta(\mathrm{ppm})$ Diastereoisomeric mixture: $\delta 1.07(\mathrm{~s}, 9 \mathrm{H}), 3.41-3.6(\mathrm{~m}$, $1 \mathrm{H}, \mathrm{NH}), 3.80(\mathrm{~s}, 3 \mathrm{H}), 3.82(\mathrm{~s}, 3 \mathrm{H}), 3.9-4.52(\mathrm{~m}, 5 \mathrm{H})$, 4.84 (d, $\left.{ }^{3} \mathrm{~J} 8.8 \mathrm{~Hz}, 1 \mathrm{H}\right), 5.97$ (bs, 2H, $\left.\mathrm{OCH}_{2} \mathrm{O}\right), 6.77-7.65$ (m, $17 \mathrm{H}$ aromatics). ${ }^{13} \mathrm{C} \mathrm{NMR}\left(75 \mathrm{MHz}, \mathrm{CDCl}_{3}\right)$ Diastereoisomeric mixture: $\delta 19.4,27,52,55.3,56.8,62.4$, $70.5,79.6,100.9,107.5,107.9,113.7,120.5,127.6,129.2$, 
129.6, 130, 132.3, 133.1, 135.4, 147.4, 147.7, 156.1, 159.

HRMS (EI, $70 \mathrm{eV}$ ) Calc. for $\mathrm{C}_{36} \mathrm{H}_{41} \mathrm{NO}_{6} \mathrm{Si}$ : 553.19207 $\left[\mathrm{M}^{+}\right.$- $t$-butyl]. Found: 553.19200 .

(土)-5-Benzo[1,3]dioxol-5-yl-4-(tert-butyl-diphenylsilanyloxymethyl)-2-oxazolidinone (32)

To a mixture of the diastereoisomeric carbamates $\mathbf{3 1 a}$ / b $(0.03 \mathrm{~g}, 0.048 \mathrm{mmol})$ and DMAP $(0.018 \mathrm{~g}, 0.144 \mathrm{mmol})$ in dry dicloromethane $(3.0 \mathrm{~mL})$ at $0{ }^{\circ} \mathrm{C}$ was slowly added trifluoromethane sulfonic anhydride $\left(\mathrm{TF}_{2} \mathrm{O}\right)(40 \mu \mathrm{L}, 0.244$ $\mathrm{mmol})$. The resulting mixture was stirred for $2 \mathrm{~h}$ at $5{ }^{\circ} \mathrm{C}$. After that time, the reaction medium was transfered to a separatory funnel and diluted with dichloromethane (10 $\mathrm{mL})$. The phases were separated and the organic one was washed with a $10 \%$ solution of $\mathrm{HCl}(2 \times 10 \mathrm{~mL})$, distilled water $(2 \times 10 \mathrm{~mL})$ and brine $(2 \times 10 \mathrm{~mL})$. The organic phase was dried over anhydrous magnesium sulfate and the solvent was evaporated. The residue was purified by flash silica gel colum chromatography (hexane/ethyl acetate $40 \%$ ) to give oxazolidinone $\mathbf{3 2}$ as a colorless oil in $30 \%$ yield.

IR $v_{\max } / \mathrm{cm}^{-1}: 3224,2929,2856,1758,1587,1493$, 1250, 1111, 702. ${ }^{1} \mathrm{H}$ NMR (300 MHz, $\mathrm{CDCl}_{3}$ ): $\delta 1.07$ (s, 9H, $\left.3 \mathrm{CH}_{3}\right), 3.70-3.80(\mathrm{~m}, 3 \mathrm{H}), 5.18\left(\mathrm{~d},{ }^{2} J 5.0 \mathrm{~Hz}\right.$, $1 \mathrm{H}), 5.44$ (bs, $1 \mathrm{H}, \mathrm{NH}), 5.98\left(\mathrm{~s}, 2 \mathrm{H}, \mathrm{OCH}_{2} \mathrm{O}\right), 6.65-$ 6.85 (m, $3 \mathrm{H}$ aromatics), 7.35-7.67 (m, $10 \mathrm{H}$ aromatics). ${ }^{13} \mathrm{C}$ NMR (75 MHz, $\mathrm{CDCl}_{3}$ ): $\delta 19.1,26.7,61.5,64.9$, 79.8, 101.4, 106.2, 108.4, 119.7, 128.1, 130.2, 132.5, 132.7, 135.6, 148.2, 148.4, 158.6. HRMS (EI, 70eV) $m / z$ Calc. for $\mathrm{C}_{27} \mathrm{H}_{29} \mathrm{NO}_{5} \mathrm{Si}\left[\mathrm{M}^{+}\right]$: 475.1815. Found: 475.1811 .

7-Hydroxymethyl-[1,3]dioxol[4,5-g]isoquinolin-5-ol (33)

To a solution of carbamate $31(0.086$ g. $0.13 \mathrm{mmol})$ in a mixture of anydrous toluene $(5.0 \mathrm{~mL})$ and dry pyridine $(0.5 \mathrm{~mL})$ at $0{ }^{\circ} \mathrm{C}$ was added, drop by drop, phosphorus oxychloride $(0.36 \mathrm{~mL}, 3.88 \mathrm{mmol})$. The resulting mixture was stirred for $2 \mathrm{~h}$ at room temperature and then refluxed for $16 \mathrm{~h}$, under an argon atmosphere. After that, the mixture was cooled to $0{ }^{\circ} \mathrm{C}$ followed by the addition of distilled water $(5.0 \mathrm{~mL})$. The phases were separated and the aqueous one was extracted with ethyl acetate. The organic phase was separated and washed with a $1 \mathrm{~mol} \mathrm{~L}^{-1}$ solution of $\mathrm{HCl}(2 \times 10 \mathrm{~mL})$, a saturated solution of $\mathrm{NaHCO}_{3}(2 \times 10 \mathrm{~mL})$, distilled water $(2 \times 10 \mathrm{~mL})$ and brine $(2 \times 10 \mathrm{~mL})$. The organic phase was then dried over anhydrous magnesium sulfate and the solvent was evaporated to provide a silylated intermediate as a yellow oil in $50 \%$ yield. This crude intermediate was dissolved in anhydrous THF and the solution was cooled to $0{ }^{\circ} \mathrm{C}$. After that a solution 1.0 mol L-1 of tetrabutylammonium fluoride in THF ( 0.3 $\mathrm{mL}, 0.3 \mathrm{mmol}$ ) was added. The resulting mixture was stirred for $2 \mathrm{~h}$ at room temperature. Then the solvent was evaporated and residue was dissolved in ethyl acetate, the organic layer was washed with a saturated solution of ammonium chloride $(2 \times 10 \mathrm{~mL})$, distilled water $(2 \times 10 \mathrm{~mL})$ and brine $(2 \times 10 \mathrm{~mL})$ and finally dried over anhydrous magnesium sulfate. The solvent was evaporated and the residue was purified by flash silica gel column chromatography (ethyl acetate/ methanol $10 \%$ ) to afford the isoquinoline $\mathbf{3 3}$ as colorless oil in $75 \%$ yield.

IR $v_{\max } / \mathrm{cm}^{-1}: 3400-3300,2920,2851,1726 ; 1588$, 1477, 1245, 1034, 883; 722; 611. 'H NMR (300 MHz, $\left.\mathrm{CDCl}_{3}\right): \delta 1.83(\mathrm{bs}, 1 \mathrm{H}, \mathrm{OH}), 4.74\left(\mathrm{~s}, 2 \mathrm{H}, \mathrm{CH}_{2}\right), 6.17(\mathrm{~s}$, $\left.2 \mathrm{H}, \mathrm{OCH}_{2} \mathrm{O}\right), 7.10(\mathrm{~s}, 1 \mathrm{H}), 7.59(\mathrm{~s}, 2 \mathrm{H}) \cdot{ }^{13} \mathrm{C}$ NMR $(75$ $\left.\mathrm{MHz}, \mathrm{CDCl}_{3}\right): \delta 66.1,102.3,102.7,103.1,119.1,123.4$, 136.8, 148, 149.3, 149.9, 152.1 .

HRMS (EI, 70eV) $\mathrm{m} / z$ Calc. for $\mathrm{C}_{11} \mathrm{H}_{9} \mathrm{NO}_{4}\left[\mathrm{M}^{+}\right]$: 219.0532. Found: 219.0491.

General procedure for protection of brominated $M B H$ adduct (34)

To a solution of $\mathrm{MBH}$ adducts $34(3-4 \mathrm{~g})$ in dry dichloromethane ( $c a .20 \mathrm{~mL}$ ) was added dry triethylamine (2.0. equiv.) and triisopropylsilyl triflate or tertbutyldimethylsilyl triflate (1.3 equiv.) at room temperature. The resulting mixture was stirred for $1 \mathrm{~h}$ at room temperature. After that, the mixture was diluted with dichloromethane $(c a .30 \mathrm{~mL})$ and the organic phase was washed with a saturated solution of $\mathrm{NaHCO}_{3}(2 \times 50 \mathrm{~mL})$ and brine $(2 \times 50 \mathrm{~mL})$, dried over anhydrous magnesium sulfate and the solvent evaporated. The residue was purified by flash silica gel column chromatography to provide the corresponding silylated ether in very good yield (for details see below).

(土) Methyl 2-[(6-bromo-benzo[1,3]dioxol-5-yl-(tert-butyldimethyl-silanyloxy)-methyl]acrylate (35)

$98 \%$ yield, a colorless viscous oil. IR $v_{\max } / \mathrm{cm}^{-1}: 2953$, 2929, 2894, 2856, 1727, 1630, 1474, 1257, 1230, 1076, 1039, 837. ${ }^{1} \mathrm{H}$ NMR (300 MHz, $\left.\mathrm{CDCl}_{3}\right): \delta-0.06(\mathrm{~s}, 3 \mathrm{H})$, $0.11(\mathrm{~s}, 3 \mathrm{H}), 0.87(\mathrm{~s}, 9 \mathrm{H}), 3.70\left(\mathrm{~s}, 3 \mathrm{H}, \mathrm{CH}_{3}\right), 5.83\left(\mathrm{t},{ }^{2} J 1.0\right.$ $\mathrm{Hz}, 1 \mathrm{H}), 5.90\left(\mathrm{~d},{ }^{2} J 1.0 \mathrm{~Hz}, 1 \mathrm{H}\right), 5.94\left(\mathrm{~d},{ }^{2} J 1.46 \mathrm{~Hz}, 1 \mathrm{H}\right)$, $5.97\left(\mathrm{~d},{ }^{2} J 1.46 \mathrm{~Hz}, 1 \mathrm{H}\right), 6.26\left(\mathrm{t},{ }^{4} J 1.0 \mathrm{~Hz}\right), 6.87$ (s, $1 \mathrm{H}$ aromatic), 6.94 (s, $1 \mathrm{H}$ aromatic). ${ }^{13} \mathrm{C}$ NMR $(75 \mathrm{MHz}$, $\left.\mathrm{CDCl}_{3}\right)$ : $\mathrm{d}-4.8,-4.5,18.1,25.9,51.8,71.5,101.6,108.8$, $112.1,113.3,124.9,134.6,143,147.2,147.5,166.1$. HRMS (EI, 70eV) $m / z$ Calc. for $\mathrm{C}_{18} \mathrm{H}_{25} \mathrm{BrO}_{5} \mathrm{Si}\left[\mathrm{M}^{+}\right]$: 428.0654. Found: 372.9949 [M - $t$-butyl]. 
(土) Methyl 2-[(6-bromo-benzo[1,3]dioxol-5-yl-(triisopropylsilanyloxy-methyl]-acrylate (36)

$87 \%$ yield, a colorless viscous oil. IR $v_{\max } / \mathrm{cm}^{-1}: 2945$, 2865, 1730, 1610, 1475, 1265, 1230, 1086, 1065, 1039, 881, 814. ' $\mathrm{H}$ NMR (300 MHz, $\left.\mathrm{CDCl}_{3}\right): \delta$ 0.92-1.17 (m, 21H), $3.70(\mathrm{~s}, 3 \mathrm{H}), 5.94\left(\mathrm{~d},{ }^{2} J 1.1 \mathrm{~Hz}, 1 \mathrm{H}\right), 5.98\left(\mathrm{~d},{ }^{2} J 1.1\right.$ $\mathrm{Hz}, 1 \mathrm{H}), 6.01(\mathrm{bs}, 1 \mathrm{H}), 6.10(\mathrm{bs}, 1 \mathrm{H}), 6.3(\mathrm{bs}, 1 \mathrm{H}), 6.92(\mathrm{~s}$, $1 \mathrm{H}$ aromatic), 6.93 (s, $1 \mathrm{H}$ aromatic). ${ }^{13} \mathrm{C}$ NMR $(75 \mathrm{MHz}$, $\left.\mathrm{CDCl}_{3}\right): \delta 12.5,18.1,51.7,71.5,101.6,108.9,112,113.4$, 124.8, 135.1, 143.3, 147.2, 147.5, 166. HRMS (ESI) $\mathrm{m} / \mathrm{z}$ Calc. for $\mathrm{C}_{21} \mathrm{H}_{31} \mathrm{BrO}_{5} \mathrm{Si}\left[\mathrm{M}^{+}\right]$: 470.1124. Found: 493.1020 $[\mathrm{M}+\mathrm{Na}]^{+}$.

General procedure for the reduction of silylated ether 35 and 36 with DIBAL-H

A solution of silyl ether $35(2.20 \mathrm{~g}, 5.12 \mathrm{mmol})$ or silyl ether $36(4.07 \mathrm{~g}, 8.64 \mathrm{mmol})$ in dry dichloromethane $(20 \mathrm{~mL})$, under an atmosphere of argon, was cooled to $-78{ }^{\circ} \mathrm{C}$ and then a solution of DIBAL-H (1.5 mol L-1, 2.4 equiv.) was slowly added. At the end, the resulting solution was stirred for $2 \mathrm{~h}$, at $-78^{\circ} \mathrm{C}$. After that time, a saturated solution of sodium acetate in distilled water was added (15-26 mL) and the temperature was allow to warm to room temperature. Then, the reaction medium was poured into a beaker containing a mixture of ethyl ether $(50-100 \mathrm{~mL})$ and a saturated solution of ammonium chloride (30-54 mL). The mixture was stirred until the formation of a gel (approximately $1 \mathrm{~h}$ ). The gel was then filtered over a pad of Celite ${ }^{\circledR}$ and the solid was washed with portions of ethyl ether $(3 \times 20 \mathrm{~mL})$. The organic phases were combined, dried over anhydrous magnesium sulfate and the solvent was evaporated. The residue was purified by flash silica gel column chromatography (hexane/ ethyl acetate $15 \%$ ) to provide the corresponding monoprotected diols $\mathbf{3 7}$ and $\mathbf{3 8}$ (for spectroscopic data see below).

\footnotetext{
(土)-[6-Bromo-benzo[1,3]dioxol-5-yl-(tert-butyl-dimethylsilanyloxy)-methyl]-2-propen-1-ol (37)

$97 \%$ yield, as a colorless oil. IR $v_{\max } / \mathrm{cm}^{-1}: 3376,2943$, 2892, 2866, 1503, 1475, 1405, 1233, 1106, 1041, 882, 683. ${ }^{1} \mathrm{H}$ NMR ( $300 \mathrm{MHz}, \mathrm{CDCl}_{3}$ ): $\delta-0.05$ (s, 3H), 0.10 (s, 3H), 0.89 (s, 9H), 1.84 (bs, 1H, OH), 4.03 (d, ${ }^{2} J 14$ $\mathrm{Hz} 1 \mathrm{H}), 4.10\left(\mathrm{~d},{ }^{2} \mathrm{~J} 14 \mathrm{~Hz}, 1 \mathrm{H}\right), 5.16(\mathrm{~s}, 1 \mathrm{H}), 5.18$ (s, $1 \mathrm{H}), 5.59(\mathrm{~s}, 1 \mathrm{H}, \mathrm{CH}), 5.96\left(\mathrm{~d},{ }^{2} J 1.0 \mathrm{~Hz}, 1 \mathrm{H}\right), 5.99\left(\mathrm{~d},{ }^{2} J\right.$ $1.0 \mathrm{~Hz}, 1 \mathrm{H}), 6.93$ (s, $1 \mathrm{H}$ aromatic), 7.0 (s, $1 \mathrm{H}$ aromatic). ${ }^{13} \mathrm{C}$ NMR $\left(75 \mathrm{MHz}, \mathrm{CDCl}_{3}\right): \delta-4.9,-4.7,18.2,25.8$, $63.4,75,101.7,108.4,112,112.3,112.5,135,147.5$, 147.6, 148.8 .
}

(土)-2-[(6-Bromo-benzo[1,3]dioxol-5-yl)-triisopropylsilanyloxy-methyl]-2-propen-1-ol (38)

$95 \%$ yield, as a colorless oil. IR $v_{\max } / \mathrm{cm}^{-1}: 3383,2943$, 2866, 1509, 1473, 1388, 1234, 1107, 1045, 942, 888, 825. ${ }^{1} \mathrm{H}$ NMR $\left(300 \mathrm{MHz}, \mathrm{CDCl}_{3}\right): \delta$ 0.92-1.16 (m, 21H), 2.02 (bs, 1H, OH), $4.0\left(\mathrm{~d},{ }^{2} J 13.6 \mathrm{~Hz}, 1 \mathrm{H}\right), 4.13\left(\mathrm{~d},{ }^{2} J 13.6 \mathrm{~Hz}\right.$, $1 \mathrm{H}), 5.19(\mathrm{~s}, 1 \mathrm{H}), 5.45(\mathrm{~s}, 1 \mathrm{H}), 5.7(\mathrm{~s}, 1 \mathrm{H}), 5.96\left(\mathrm{~d},{ }^{2} J 1.5\right.$ $\mathrm{Hz}, 1 \mathrm{H}), 6.0\left(\mathrm{~d},{ }^{2} J 1.5 \mathrm{~Hz}, 1 \mathrm{H}\right), 6.93$ (s, $1 \mathrm{H}$ aromatic), 7.09 (s, $1 \mathrm{H}$ aromatic). $\left.{ }^{13} \mathrm{C} \mathrm{NMR} \mathrm{(75} \mathrm{MHz}, \mathrm{CDCl}_{3}\right): \delta 12$, $18.8,19.9,62.7,75.2,101.7,108.1,111.9,112,112.1$, 135.4, 147.5, 147.6, 148.6. HRMS (ESI) $\mathrm{m} / z$ Calc. for $\mathrm{C}_{20} \mathrm{H}_{31} \mathrm{BrO}_{4} \mathrm{Si}\left[\mathrm{M}^{+}\right]$: 442.1175. Found: 465.1071 [M+Na $]^{+}$.

(土)-5-Bromo-6-[1-(tert-butyl-dimethyl-silanyloxy)-2-(tertbutyl-diphenyl-silanyloxymethyl)-allyl]-benzo[1,3]dioxol (39)

To a mixture of mono protected diol $37(2.0 \mathrm{~g}, 5 \mathrm{mmol})$, $\mathrm{N}, \mathrm{N}$-dimethylaminopyridine (DMAP) $(0.015 \mathrm{~g}, 0.125$ mmol) and dry triethylamine $(1.4 \mathrm{~mL}, 10 \mathrm{mmol})$ in anhydrous dichloromethane $(20 \mathrm{~mL})$ was added tertbutyldimethyl silyl chloride $(2.0 \mathrm{~mL}, 7.5 \mathrm{mmol})$. The resulting solution was stirred for $16 \mathrm{~h}$, at room temperature. After that, the mixture was diluted with hexane $(50 \mathrm{~mL})$ and the organic layer was washed with a saturated solution of $\mathrm{NaHCO}_{3}$. The organic layer was separated, dried over anhydrous sodium sulfate and the solvent was evaporated. The residue was purified by flash silica gel column chromatography (hexane/ethyl acetate $5 \%$ ) to provide 39 as colorless viscous oil in $93 \%$ yield.

IR $v_{\max } / \mathrm{cm}^{-1}: 2954,2929,2856,1613,1473,1232$, 1109, 1069, 1040, 874, 836. ${ }^{1} \mathrm{H}$ NMR (300 MHz, $\left.\mathrm{CDCl}_{3}\right)$ : $\delta-0.09(\mathrm{~s}, 3 \mathrm{H}), 0.03(\mathrm{~s}, 3 \mathrm{H}), 0.84(\mathrm{~s}, 9 \mathrm{H}), 1.05(\mathrm{~s}, 9 \mathrm{H})$, $4.13(\mathrm{~m}, 2 \mathrm{H}), 5.19(\mathrm{~s}, 1 \mathrm{H}), 5.33(\mathrm{~s}, 1 \mathrm{H}), 5.50(\mathrm{~s}, 1 \mathrm{H}), 5.94$ (d, $\left.{ }^{2} J 1.5 \mathrm{~Hz}, 2 \mathrm{H}\right), 6.88(\mathrm{~s}, 1 \mathrm{H}$ aromatic), $6.9(\mathrm{~s}, 1 \mathrm{H}$ aromatic), 7.37-7.64 (m, 10H aromatics). ${ }^{13} \mathrm{C}$ NMR (75 $\left.\mathrm{MHz}, \mathrm{CDCl}_{3}\right): \delta-4.9,-4.7,18.2,19.3,25.8,26.8,63.5$, 73.6, 101.5, 108.5, 110, 111.7, 112.4, 127.4, 129.4, 133.5, 135.3, 135.5, 147.3, 147.3, 149.2. HRMS (ESI) $\mathrm{m} / \mathrm{z}$ Calc. for $\mathrm{C}_{33} \mathrm{H}_{43} \mathrm{BrO}_{4} \mathrm{Si}_{2}\left[\mathrm{M}^{+}\right]$: 638.1883. Found: $661.1779[\mathrm{M}+$ $\mathrm{Na}]^{+}$.

( \pm )-[1,6-Bromo-benzo[1,3]dioxol-5-yl-2-(4-methoxybenzyloxymethyl)-allyloxy] triisopropylsilane (40)

To a solution of $\mathbf{3 8}(0.89 \mathrm{~g}, 2 \mathrm{mmol})$ in dry dichloromethane $(5.0 \mathrm{~mL})$ was added, at room temperature, $p$-methoxybenzyl trichloroacetoimidate $(0.85 \mathrm{~g}$, $3 \mathrm{mmol}$ ) and a catalytic amount of camphorsulfonic acid $(0.023 \mathrm{~g}, 0.1 \mathrm{mmol})$. The resulting solution was stirred for $18 \mathrm{~h}$ at room temperature. Then, the reaction medium was diluted with ethyl ether $(25 \mathrm{~mL})$, washed with a saturated solution of $\mathrm{NaHCO}_{3}(2 \times 10 \mathrm{~mL})$, distilled water $(2 \times$ 
$10 \mathrm{~mL})$ and brine $(2 \times 10 \mathrm{~mL})$. The organic phase was dried over anhydrous magnesium sulfate and the solvent was evaporated. The residue was purified by flash silica gel column chromatography (hexane/ethyl acetate $5 \%$ ) to provide $\mathbf{4 0}$ as a colorless oil in $98 \%$ yield.

IR $v_{\text {max }} / \mathrm{cm}^{-1}: 3422,2943,2865,1513,1474,1247,1039$. ${ }^{1} \mathrm{H}$ NMR $\left(300 \mathrm{MHz}, \mathrm{CDCl}_{3}\right): \delta$ 0.92-1.14 $(\mathrm{m}, 21 \mathrm{H}, 3 \times$ isopropyl), 3.81(s, 3H), 3.92 (s, 2H), 4.37 (s, 2H), 5.26 (bs, $1 \mathrm{H}), 5.56(\mathrm{bs}, 1 \mathrm{H}), 5.68(\mathrm{bs}, 1 \mathrm{H}), 5.94\left(\mathrm{~d},{ }^{2} J 1.5 \mathrm{~Hz}, 1 \mathrm{H}\right)$, $5.98\left(\mathrm{~d},{ }^{2} J 1.5 \mathrm{~Hz}, 1 \mathrm{H}\right), 6.85\left(\mathrm{~d},{ }^{3} J 8.8 \mathrm{~Hz}, 2 \mathrm{H}\right), 6.92(\mathrm{~s}$, 1H), $7.02(\mathrm{~s}, 1 \mathrm{H}), 7.2\left(\mathrm{~d},{ }^{3} \mathrm{~J} 8.8 \mathrm{~Hz}, 2 \mathrm{H}\right) \cdot{ }^{13} \mathrm{C}$ NMR $(75$ $\left.\mathrm{MHz}, \mathrm{CDCl}_{3}\right): \delta 12.3,18.1,55.3,68.9,71.7,74,101.5$, $108.4,111.7,111.8,113.5,113.7,129,129.6,130.4,135.9$, 146.9, 147.3, 158.8. HRMS (ESI) $\mathrm{m} / \mathrm{z}$ Calc. for $\mathrm{C}_{28} \mathrm{H}_{39} \mathrm{BrO}_{5} \mathrm{Si}\left[\mathrm{M}^{+}\right]: 562.1750$. Found: $585.1645[\mathrm{M}+\mathrm{Na}]^{+}$.

(土)-5-Bromo-6-[2-tert-butyl-diphenyl-silanyloxymethyl)1-triisopropyl-silanyloxy-allyl]-benzo[1,3]dioxol (41)

To a mixture of $38(0.887 \mathrm{~g}, 2 \mathrm{mmol}), N, N$ dimethylaminopyridine (DMAP) $(0.006 \mathrm{~g}, 0.05 \mathrm{mmol})$ and dry triethylamine $(0.56 \mathrm{~mL}, 4 \mathrm{mmol})$ in dry dichloromethane $(8.0 \mathrm{~mL})$, at room temperature and under an atmosphere of argon, was added tert-butyldiphenylsilane chloride $(0.8 \mathrm{~mL}, 3 \mathrm{mmol})$. The resulting mixture was stirred for $16 \mathrm{~h}$ at room temperature and under an argon atmosphere. After that, the reaction medium was diluted with hexane $(50 \mathrm{~mL})$ and the organic layer was washed with a saturated solution of $\mathrm{NaHCO}_{3}(2 \times 20 \mathrm{~mL})$ and dried over anhydrous sodium sulfate. The solvent was evaporated and the residue was purified by flash silica gel column chromatography (hexane/ ethyl acetate $2 \%$ ) to afford $\mathbf{4 1}$ as a colorless oil in $91 \%$ yield.

IR $v_{\text {max }} / \mathrm{cm}^{-1}: 3070,3051,2939,2862,1504 ; 1473,1388$, 1234, 1107, 1045, 937, 883, 825, 702. ${ }^{1} \mathrm{H}$ NMR (500 MHz, $\left.\mathrm{CDCl}_{3}\right): \delta 0.98-1.08$ (m, $30 \mathrm{H}, t$-butyl $+3 \times$ isopropyl), 4.17 $(\mathrm{s}, 2 \mathrm{H}), 5.42(\mathrm{bs}, 1 \mathrm{H}), 5.53(\mathrm{bs}, 1 \mathrm{H}) 5.65(\mathrm{bs}, 1 \mathrm{H}), 5.93(\mathrm{~d}$, $\left.{ }^{2} J 1.5 \mathrm{~Hz}, 1 \mathrm{H}\right), 5.97\left(\mathrm{~d},{ }^{2} J 1.5 \mathrm{~Hz}, 1 \mathrm{H}\right), 6.84$ (s, $1 \mathrm{H}$ aromatic), 6.9 (s, $1 \mathrm{H}$ aromatic), 7.36-7.68 (m, $10 \mathrm{H}$ aromatics). ${ }^{13} \mathrm{C}$ NMR (125 MHz, $\left.\mathrm{CDCl}_{3}\right): \delta 12.2,18,19.2,26.8,62.9,73.9$, 101.5, 108.4, 109.5, 111.7, 112.3, 127.5, 129.5, 133.6, 133.7, 135.4, 135.5, 136.1, 147.4, 147.4, 149.6. HRMS (EI, $70 \mathrm{eV}) \mathrm{m} / z$ calc. for $\mathrm{C}_{36} \mathrm{H}_{49} \mathrm{BrO}_{4} \mathrm{Si}_{2}\left[\mathrm{M}^{+}\right]: 680.2353$. Found: 680.2328 .

General procedure for hydroboration reaction of 39, 40 and 41

To a solution of compounds 39 (2.98 g, $4.66 \mathrm{mmol})$, 40 (1.1 g, $1.96 \mathrm{mmol})$ or $41(1.24 \mathrm{~g}, 1.82 \mathrm{mmol})$ in anhydrous THF (15, 15 or $5 \mathrm{~mL}$, respectively) was slowly added, at $0{ }^{\circ} \mathrm{C}$, a solution of 9-BBN in THF $\left(0.5 \mathrm{~mol} \mathrm{~L}^{-1}\right)$
( 5 equiv.). The reaction was then stirred for $16 \mathrm{~h}$ at room temperature. After that, the reaction was cooled to $0{ }^{\circ} \mathrm{C}$ and a $3.0 \mathrm{~mol} \mathrm{~L}^{-1}$ solution of $\mathrm{NaOH}(55,16$ and $15 \mathrm{~mL}$, respectively for the reactions with $\mathbf{3 9 , 4 0}$ and $\mathbf{4 1}$ ) was added, followed by the addition of $30 \% \mathrm{H}_{2} \mathrm{O}_{2}(55,15$ and $16 \mathrm{~mL}$, respectively). The mixture was stirred for $45 \mathrm{~min}$ at $0{ }^{\circ} \mathrm{C}$ and $45 \mathrm{~min}$ at room temperature. Then, a saturated solution of $\mathrm{NaHCO}_{3}$ was added and the mixture was transferred to a separatory funnel and diluted with dichloromethane $(80 \mathrm{~mL})$. The phases were separated amd the organic one was washed with $\mathrm{NaHCO}_{3}(2 \times 20 \mathrm{~mL})$, distilled water $(2 \times 20 \mathrm{~mL})$ and brine $(2 \times 20 \mathrm{~mL})$, dried over anhydrous magnesium sulfate and the solvent was evaporated. The residue was purified by flash silica gel column chromatography to furnish the corresponding alcohol (see the analytical and spectroscopic data for each compound below).

( \pm -3-(6-Bromo-benzo[1,3]dioxol-5-yl)-3-(tert-butyldimethylsilanyloxy)-2-(tert-butyl-diphenyl silanyloxymethy)-1-propanol (42a/b-syn/anti)

Colorless oil, $82 \%$ yield. IR $v_{\max } / \mathrm{cm}^{-1}: 3425,3070$, 2930, 2866, 1503, 1473, 1391, 1238, 1112, 1045, 937. 879. ${ }^{1} \mathrm{H}$ NMR (500 $\mathrm{MHz}, \mathrm{CDCl}_{3}$ ) Diastereoisomeric mixture: $\delta-0.19$ (s, 3H), 0.01 (s, 3H), 0.85 (s, 9H), 1.06 (s, 9H), $1.97(\mathrm{~m}, 1 \mathrm{H}), 3.73-3.90(\mathrm{~m}, 4 \mathrm{H}), 5.21$ (d, ${ }^{3} \mathrm{~J} 3.7$ $\mathrm{Hz}, 1 \mathrm{H}), 5.97$ (d, $\left.{ }^{2} J 1.5 \mathrm{~Hz}, 1 \mathrm{H}\right), 5.99\left(\mathrm{~d},{ }^{2} J 1.5 \mathrm{~Hz}, 1 \mathrm{H}\right)$, 6.91 (s, 1H aromatic), 6.99 (s, 1H aromatic), 7.39-7.66 (m, $10 \mathrm{H}$ aromatics). ${ }^{13} \mathrm{C}-\mathrm{NMR}\left(125 \mathrm{MHz}, \mathrm{CDCl}_{3}\right)$ Diastereoisomeric mixture: $\delta-5.4,-4.8,14.14,17.9,19.1$, 25.7, 26.5, 26.9, 48.1, 60.3, 61.8, 63.9, 74, 101.7, 108.3, 111.6, 112.2, 127.6, 127.7, 129.6, 129.7, 133.2, 133.3, 134.7, 135.4, 135.5, 135.6, 147.4; 147.5. HRMS (EI, 70 eV) $\mathrm{m} / z$ Calc. for $\mathrm{C}_{33} \mathrm{H}_{45} \mathrm{BrO}_{5} \mathrm{Si}\left[\mathrm{M}^{+}\right]: 656.1989$. Found: 656.1926 .

( \pm )-3-(6-Bromo-benzo[1,3]dioxol-5-yl)-2-(tert-butyldiphenyl-silanyloxy-methyl)-3-triisopropylsilanyloxy-1propanol (43a/b-syn/anti)

Colorless oil (mixture of diastereoisomers), $77 \%$ yield. IR $v_{\max } / \mathrm{cm}^{-1}: 3406,2940,2865,1612,1513,1476,1240$, 1170, 1105, 1037, 934. ${ }^{1} \mathrm{H}$ NMR (500 MHz, $\mathrm{CDCl}_{3}$ ) Major diastereoisomer $( \pm)-$ syn-43a: d 0.95-1.06 (m, 21H, $3 \times$ isopropyl), $2.08(\mathrm{~m}, 1 \mathrm{H}), 2.4(\mathrm{bs}, 1 \mathrm{H}, \mathrm{OH}), 3.72-3.78(\mathrm{~m}$, $2 \mathrm{H}), 3.81(\mathrm{~s}, 3 \mathrm{H}), 3.82-3,87(\mathrm{~m}, 2 \mathrm{H}), 4.44(\mathrm{dd}, J 15.3$ and $11.7 \mathrm{~Hz}, 2 \mathrm{H}), 5.25\left(\mathrm{~d},{ }^{3} J 5.5 \mathrm{~Hz}, 1 \mathrm{H}\right), 6.01(\mathrm{~d}, J 1.5 \mathrm{~Hz}$, $2 \mathrm{H}), 6.87(\mathrm{~d}, J 8.7 \mathrm{~Hz}, 2 \mathrm{H}$ aromatics $), 6.94(\mathrm{~s}, 1 \mathrm{H}$ aromatic), 7.05 (s, $1 \mathrm{H}$ aromatic), $7.24(\mathrm{~d}, J 8.7 \mathrm{~Hz}, 2 \mathrm{H}$ aromatics). Minor diastereoisomer $( \pm)$-anti-43b: $\delta 0.95$ $1.06(\mathrm{~m}, 21 \mathrm{H}, 3 \times$ isopropyl), $2.27(\mathrm{~m}, 1 \mathrm{H}), 2.4(\mathrm{bs}, 1 \mathrm{H}$, $\mathrm{OH}), 3.58-3.7(\mathrm{~m}, 2 \mathrm{H}), 3.8(\mathrm{~s}, 3 \mathrm{H}), 3.82-3.87(\mathrm{~m}, 2 \mathrm{H})$, 
$4.44(\mathrm{bs}, 2 \mathrm{H}), 5.35\left(\mathrm{~d},{ }^{3} J 7 \mathrm{~Hz}, 1 \mathrm{H}\right), 6.01(\mathrm{~d}, J 1.5 \mathrm{~Hz}, 2 \mathrm{H})$, 6.87 (d, $J 8.7 \mathrm{~Hz}, 2 \mathrm{H}$ aromatics), 6.94 (s, $1 \mathrm{H}$ aromatic), 7.05 (s, $1 \mathrm{H}$ aromatic), 7.24 (d, $J 8.7 \mathrm{~Hz}, 2 \mathrm{H}$ aromatics). ${ }^{13} \mathrm{C}$ NMR $\left(125 \mathrm{MHz}, \mathrm{CDCl}_{3}\right.$ ) Major diastereoisomer $( \pm$ )syn-43a: $\delta 12.5,18,48.6,55.3,63.9,69.9,72.9,74.1$, 101.7, 108.5, 112, 112.3, 113.7, 129.2, 129.9, 130.1, 135.6, 147.4, 159. Minor diastereoisomer ( \pm -anti-43b: $\delta$ 12.5, $18,47.6,55.3,62.3,70.3,73.1,73.3,101.7,108.4,112.1$, 112.3, 113.6, 129, 129.4, 130.1, 135.9, 147.4, 159. HRMS (ESI) $m / z$ Calc. for $\mathrm{C}_{28} \mathrm{H}_{41} \mathrm{BrNaO}_{6} \mathrm{Si}^{+}[\mathrm{M}+\mathrm{Na}]^{+}: 603.1748$. Found: 603.1739 .

(土)-3-(6-Bromo-benzo[1,3]dioxol-5-yl)-2-(tert-butyldiphenyl-silanyloxy-methyl)-3-triisopropylsilanyloxy-1propanol (44)

Colorless oil, $77 \%$ yield. IR $v_{\max } / \mathrm{cm}^{-1}: 3425,3070$, 2930, 2866, 1504, 1473, 1426, 1391, 1238, 1111, 1045, 937, 879, 706. ${ }^{1} \mathrm{H}$ NMR (300 $\mathrm{MHz}, \mathrm{CDCl}_{3}$ ) Major diastereoisomer ( \pm )-syn-44: $\delta$ 0.80-1.01 (m, 21H, $3 \times$ isopropyl), $1.05(\mathrm{~s}, 9 \mathrm{H}, t$-butyl), $1.90(\mathrm{~m}, 1 \mathrm{H}), 2.0$ (bs, $1 \mathrm{H}, \mathrm{OH}), 3.78-3.90(\mathrm{~m}, 4 \mathrm{H}), 5.31\left(\mathrm{~d},{ }^{3} J 3.7 \mathrm{~Hz}, 1 \mathrm{H}, \mathrm{CH}\right)$, $5.97\left(\mathrm{~d},{ }^{2} J 1.5 \mathrm{~Hz}, 1 \mathrm{H}\right), 6.01$ (d, $\left.{ }^{2} J 1.5 \mathrm{~Hz}, 1 \mathrm{H}\right), 6.91(\mathrm{~s}$, $1 \mathrm{H}$ aromatic), 7.03 (s, 1H aromatic), 7.37-7.68 (m, 10H aromatics). ${ }^{13} \mathrm{C}$ NMR $\left(75 \mathrm{MHz} . \mathrm{CDCl}_{3}\right.$ ) Major diastereoisomer $( \pm)-40 a: ~ \delta 12.6,18,19.2,27,48.9,61.9,64.5$, 74.4, 101.7, 108.4, 111.7, 112.2, 127.6, 129.6, 133.2, 134.7, 135.5, 135.8, 147.4. HRMS (ESI) $\mathrm{m} / z$ Calc. for $\mathrm{C}_{36} \mathrm{H}_{51} \mathrm{BrNaO}_{6} \mathrm{Si}_{2}{ }_{2}^{+}[\mathrm{M}+\mathrm{Na}]^{+}:$721.2351. Found: 721.2347.

General procedure for the preparation of aldehydes of 45,46 and 47

A mixture of alcohol 42 (3.67 g, $5.58 \mathrm{mmol})$, alcohol $43(0.8 \mathrm{~g}, 1.37 \mathrm{mmol})$ or alcohol $44(0.95, \mathrm{~g}, 1.40 \mathrm{mmol})$, $\mathrm{N}$-methyl-morpholine oxide (NMO) (1.5 equiv.), tetrapropylammonium perruthenate $(10 \mathrm{~mol} \%)$, molecular sieves $4 \AA$ (previously triturated and activated, $0.5 \mathrm{~g} \mathrm{mmol}^{-1}$ ) in anhydrous dichloromethane $\left(4 \mathrm{~mL} \mathrm{mmol}^{-1}\right)$ was stirred for $1 \mathrm{~h}$ at room temperature. After that, the reaction medium was filtered on a silica gel column and the solid was washed twice with dichloromethane. Filtrates were combined and the solvent was evaporated. The residue was used in the next step without any additional purification.

(土)-3-(6-Bromo-benzo[1,3]dioxol-5-yl)-3-(tert-butyldimethyl-silanyloxy)-2-(tert-butyl-diphenyl-silanyloxymethyl)-propionaldehyde (45)

Colorless oil, 98\% yield. IR $v_{\max } / \mathrm{cm}^{-1}: 3070,2956$, 2930, 2890, 2857, 1727, 1475, 1239, 1112, 1099, 837, 702. ${ }^{1} \mathrm{H}$ NMR (300 MHz, $\mathrm{CDCl}_{3}$ ) Mixture of diastereo- isomers: $\delta-0.19(\mathrm{~s}, 3 \mathrm{H}), 0.05(\mathrm{~s}, 3 \mathrm{H}), 0.83(\mathrm{~s}, 9 \mathrm{H}), 1.03$ (s, 9H), $2.72(\mathrm{~m}, 1 \mathrm{H}), 2.81(\mathrm{~m}, 1 \mathrm{H}, \mathrm{CH}), 3.74(\mathrm{~m}, 1 \mathrm{H}$, $\left.\mathrm{CH}_{2}\right), 3.92(\mathrm{~m}, 1 \mathrm{H}$, minor diastereoisomer $), 4.06(\mathrm{~m}, 1 \mathrm{H}$, $\left.\mathrm{CH}_{2}\right), 4.22(\mathrm{~m}, 1 \mathrm{H}$, minor diastereoisomer $), 5.38\left(\mathrm{~d},{ }^{3} \mathrm{~J}\right.$ $5.1 \mathrm{~Hz}, 1 \mathrm{H}), 5.46\left(\mathrm{~d},{ }^{3} J 6.2 \mathrm{~Hz}, 1 \mathrm{H}\right.$, minor diastereoisomer), $5.91\left(\mathrm{~d},{ }^{2} J 1.5 \mathrm{~Hz}, 1 \mathrm{H}\right), 5.97\left(\mathrm{~d},{ }^{2} J 1.5\right.$ $\mathrm{Hz}, 1 \mathrm{H}), 6.91$ (s, 1H aromoatic), 6.93 (s, 1H aromatic), 7.32-7.62 (m, $10 \mathrm{H}$ aromatics), $9.75\left(\mathrm{~d},{ }^{3} J 3.3 \mathrm{~Hz}, 1 \mathrm{H}\right.$, $\mathrm{C}(\mathrm{O}) \mathrm{H}) \cdot{ }^{13} \mathrm{C}$ NMR $\left(75 \mathrm{MHz}, \mathrm{CDCl}_{3}\right)$ Mixture of diastereoisomers: $\delta-5.4,-5.3,-4.7,-4.6,17.9,18,19.1$, 25.6, 26.7, 59.8, 60.9, 70.6, 101.7, 102.8, 108.3, 111.4, $112.2,127.6,127.6,127.7,129.6,129.7,133,133.1$, 134.4, 135.5, 135.6, 147.5, 147.7, 202.7, 202.8. HRMS (ESI) $\mathrm{m} / z$ Calc. for $\mathrm{C}_{33} \mathrm{H}_{43} \mathrm{BrNaO}_{5} \mathrm{Si}^{+}[\mathrm{M}+\mathrm{Na}]^{+}$: 677.1725. Found: 677.1718.

(土)-3-(6-Bromo-benzo[1,3]dioxol-5-yl)-2-(4-methoxybenzyloxymethyl)-3-triisopropylsilanyloxy-propionaldehyde (46)

Colorless oil, $85 \%$ yield. IR $v_{\max } / \mathrm{cm}^{-1}: 2944,2867$, 1752, 1613, 1513, 1476, 1247; 1084, 1039, 934, 882, 828, 683. ${ }^{1} \mathrm{H}$ NMR $\left(500 \mathrm{MHz}, \mathrm{CDCl}_{3}\right.$ ) Mixture of diastereoisomers: $\delta 0.95-1.06(\mathrm{~m}, 21 \mathrm{H}, 3 \times$ isopropyl), $3.58(\mathrm{~m}$, $1 \mathrm{H}), 3.68(\mathrm{~m}, 1 \mathrm{H}), 3.81(\mathrm{~s}, 3 \mathrm{H}), 4.4(\mathrm{~m}, 2 \mathrm{H}), 5.57\left(\mathrm{~d},{ }^{3} \mathrm{~J}\right.$ $5.5 \mathrm{~Hz}, 1 \mathrm{H}$, major diastereoisomer), $5.67\left(\mathrm{~d},{ }^{3} J 4.3 \mathrm{~Hz}\right.$, $1 \mathrm{H}$, minor diastereoisomer), $5.96\left(\mathrm{~d},{ }^{2} J 1.2 \mathrm{~Hz}, 1 \mathrm{H}\right.$, minor diastereoisomer), 5.98 (d, $\left.{ }^{2} J 1.2 \mathrm{~Hz}, 1 \mathrm{H}\right), 6.01\left(\mathrm{~d},{ }^{2} J 1.5\right.$ $\mathrm{Hz}, 1 \mathrm{H}$, minor diastereoisomer), $6.02\left(\mathrm{~d},{ }^{2} J 1.5 \mathrm{~Hz}, 1 \mathrm{H}\right)$, 6.83-7.21 (m, 6H aromatics), $9.83\left(\mathrm{~d},{ }^{3} J 3.1 \mathrm{~Hz}\right.$, $1 \mathrm{H}, \mathrm{C}(\mathrm{O}) \mathrm{H}) \cdot{ }^{13} \mathrm{C}$ NMR $\left(125 \mathrm{MHz}, \mathrm{CDCl}_{3}\right.$ ) Major diastereoisomer: $\delta$ 12.4, 17.9, 55.2, 58.9, 66.4, 72.1, 72.8, 101.9, 108.3, 112.2, 113.5, 113.7, 129.1, 130, 135.1, 147.6, 147.9, 159.1, 202.5. Minor diastereoisomer: $\delta 12.3,17.9,55.2$, 59.7, 64.8, 71.5, 72.9, 101.8, 108.4, 112, 113.4, 113.7, 129.2, 130, 134.8, 147.5, 147.8, 159, 202.4. HRMS (ESI) $m / z$ Calc. for $\mathrm{C}_{28} \mathrm{H}_{39} \mathrm{BrNaO}_{6} \mathrm{Si}[\mathrm{M}+\mathrm{Na}]^{+}: 601.1591$. Found: 601.1579 .

(土)-3-(6-Bromo-benzo[1,3]dioxol-5-yl)-2-(tert-butyldiphenyl-silanyloxy-methyl)-3-triisopropylsilanyloxypropionaldehyde (47)

Colorless oil, 95\% yield. IR $v_{\max } / \mathrm{cm}^{-1}: 2944,2866$, 2892, 1727, 1503, 1475, 1239, 1112, 1039, 882, 702. ${ }^{1} \mathrm{H}$ NMR (300 MHz, $\left.\mathrm{CDCl}_{3}\right): \delta 0.92-1.04(\mathrm{~m}, 30 \mathrm{H}, t$-butyl + $3 \times$ isopropyl), $2.82(\mathrm{~m}, 1 \mathrm{H}), 3.78\left(\mathrm{dd},{ }^{2} J 10.4 \mathrm{~Hz},{ }^{3} J 5.5\right.$ $\mathrm{Hz}, 1 \mathrm{H}), 4.1\left(\mathrm{dd},{ }^{2} J 10.4 \mathrm{~Hz},{ }^{3} J 5.5 \mathrm{~Hz}\right), 5.52\left(\mathrm{~d},{ }^{3} J 5.1 \mathrm{~Hz}\right.$, $1 \mathrm{H}, \mathrm{CH}), 5.97\left(\mathrm{~d},{ }^{2} J 1.5 \mathrm{~Hz}, 1 \mathrm{H}\right), 6.0\left(\mathrm{~d},{ }^{2} J 1.5 \mathrm{~Hz}, 1 \mathrm{H}\right)$, 6.91 (s, 1H aromatic), 6.93 (s, $1 \mathrm{H}$ aromatic), 7.36-7.63 (m, $10 \mathrm{H}$ aromatics), $9.82\left(\mathrm{~d},{ }^{3} \mathrm{~J} 3.3 \mathrm{~Hz}, 1 \mathrm{H}, \mathrm{C}(\mathrm{O}) \mathrm{H}\right) \cdot{ }^{13} \mathrm{C}$ NMR (75 MHz, $\left.\mathrm{CDCl}_{3}\right): \delta 12.6,18,19.2,26.9,61.1,61.6$, $72,101.8,108.3,111.8,112.2,127.6,129.6,133.1,134.7$, 
135.5, 147.4, 147.7, 202.5. HRMS (ESI) $\mathrm{m} / z$ Calc. for $\mathrm{C}_{36} \mathrm{H}_{49} \mathrm{BrNaO}_{5} \mathrm{Si}_{2}[\mathrm{M}+\mathrm{Na}]^{+}:$719.2194. Found: 719.2183.

General procedure for the preparation of acids $\mathbf{4 8 , 4 9}$ and 50

A mixture of aldehyde $\mathbf{4 5}(1.58,2.41 \mathrm{mmol})$, aldehyde $46(0.63,1.09 \mathrm{mmol})$ or aldehyde $47(0.83 \mathrm{~g}, 1.19 \mathrm{mmol})$ in $t$-butanol $(20 \mathrm{~mL}$ for $\mathbf{4 5}$ and $10 \mathrm{~mL}$ for $\mathbf{4 6}$ and $\mathbf{4 7})$ and 2-methyl-but-2-ene (48.4 equiv.) was cooled to $0{ }^{\circ} \mathrm{C}$. Then a solution of $\mathrm{NaClO}_{2}$ (9.2 equiv.) and $\mathrm{NaH}_{2} \mathrm{PO}_{4}$ (6.9 equiv.) dissolved in distilled water $(6 \mathrm{~mL}$ for $\mathbf{4 5}$ and $2.5 \mathrm{~mL}$ for aldehydes 46 and 47 ) was added. The resulting mixture was stirred for $1 \mathrm{~h}$, at room temperature. After that, the volatile components were evaporated and the residue was dissolved in distilled water $(20 \mathrm{~mL})$, which was acidified to $\mathrm{pH} 3$ with a $10 \%$ solution of $\mathrm{HCl}$. The acidified aqueous phase was then extracted with ethyl ether $(2 \times 20 \mathrm{~mL})$. The organic phase was dried over anhydrous magnesium sulfate and the solvent was evaporated. The residue was purified by flash silica gel column chromatography (hexane/ethyl acetate $20 \%$ ) to furnish the corresponding acids. The yields and spectroscopic data for each acid are shown below.

(土)-3-(6-Bromo-benzo[1,3]dioxol-5-yl)-3-(tert-butyldimethyl-silanyloxy)-2-(tert-butyl-diphenyl-silanyloxymethyl)-propanoic acid (48)

Colorless oil, 92\% yield. IR $v_{\max } / \mathrm{cm}^{-1}: 3530,3070$, 3049, 2955, 2931, 2886, 2857, 1712, 1504, 1478, 1428, 1239, 1113, 1076, 1039, 934, 839, 777, 702. ${ }^{1} \mathrm{H}$ NMR $\left(300 \mathrm{MHz}, \mathrm{CDCl}_{3}\right): \delta-0.2$ (s, 3H), 0.05 (s, 3H), 0.82 (s, 9H), $1.01(\mathrm{~s}, 9 \mathrm{H}), 2.96(\mathrm{~m}, 1 \mathrm{H}), 3.48\left(\mathrm{dd},{ }^{2} J 10.3 \mathrm{~Hz},{ }^{3} \mathrm{~J}\right.$ $5.5 \mathrm{~Hz}, 1 \mathrm{H}), 4.03\left(\mathrm{t},{ }^{2} J 9.3 \mathrm{~Hz}, 1 \mathrm{H}\right), 5.26\left(\mathrm{~d},{ }^{3} J 7.7 \mathrm{~Hz}\right.$, 1H), $5.97\left(\mathrm{~d},{ }^{2} J 1.5 \mathrm{~Hz}, 1 \mathrm{H}\right), 5.99\left(\mathrm{~d},{ }^{2} J 1.5 \mathrm{~Hz}, 1 \mathrm{H}\right), 6.83$ (s, $1 \mathrm{H}$ aromatic), 6.87 (s, $1 \mathrm{H}$ aromatic), 7.33-7.6 (m, 10H aromatics). ${ }^{13} \mathrm{C}$ NMR $\left(75 \mathrm{MHz}, \mathrm{CDCl}_{3}\right): \delta-5.2,-4.7,18$, 19.2, 25.7, 26.8, 58.3, 62.1, 71.6, 101.6, 108, 111.7, 112.2, 127.5, 129.4, 132.9, 134.6, 135.4, 147.4, 147.6, 177.5. HRMS (ESI) $m / z$ Calc. for $\mathrm{C}_{33} \mathrm{H}_{43} \mathrm{BrNaO}_{6} \mathrm{Si}_{2}[\mathrm{M}+\mathrm{Na}]^{+}$: 693.1674. Found: 693.1661.

(土)-3-(6-Bromo-benzo[1,3]dioxol-5-yl)-2-(4-methoxybenzyloxymethyl)-3-triisopropylsilanyloxy-propanoic acid (49)

Yellow tinged oil, $80 \%$ yield. IR $v_{\text {max }} / \mathrm{cm}^{-1}: 3430,2944$, 2867, 1712, 1611, 1513, 1476, 1408, 1389, 1242, 1173, 1088, 882. ${ }^{1} \mathrm{H}$ NMR (300 MHz, $\mathrm{CDCl}_{3}$ ) Diastereoisomers mixture: $\delta 0.95-1.02(\mathrm{~m}, 21 \mathrm{H}, 3 \times$ isopropyl $), 3.12(\mathrm{~m}$, $1 \mathrm{H}), 3.37-3.78(\mathrm{~m}, 2 \mathrm{H}), 3.81(\mathrm{~s}, 3 \mathrm{H}), 4.39(\mathrm{~m}, 2 \mathrm{H}), 5.48$ (d, ${ }^{3} J 6.9 \mathrm{~Hz}, 1 \mathrm{H}$ major diastereoisomer), 6.01(d, ${ }^{2} J 1.5$
$\mathrm{Hz}, 2 \mathrm{H}), 6.82-7.26$ (m, 6H, aromatics). ${ }^{13} \mathrm{C} \mathrm{NMR}(75 \mathrm{MHz}$, $\mathrm{CDCl}_{3}$ ) Diastereoisomers mixture: $\delta 12.4,15.5,50.7,55.3$, 71.6, 78.6, 101.7, 108.9, 112.4, 113.1, 113.6, 128.2, 128.5, 135.1, 136.6, 146.8, 144.5, 146.8, 159.8, 179.7. HRMS (ESI) $m / z$ Calc. for $\mathrm{C}_{28} \mathrm{H}_{39} \mathrm{BrNaO}_{7} \mathrm{Si}[\mathrm{M}+\mathrm{Na}]^{+}: 617.1541$. Found: 617.1522 .

( \pm )-3-(6-Bromo-benzo[1,3]dioxol-5-yl)-2-(tert-butyldiphenyl-silanyloxy-methyl)-3-triisopropylsilanyloxypropanoic acid (50)

Colorless oil, $85 \%$ yield. IR $v_{\max } / \mathrm{cm}^{-1}: 3359,2943$, 2866, 1712, 1473, 1238, 1149, 1111, 937, 883. 'H NMR $\left(300 \mathrm{MHz}, \mathrm{CDCl}_{3}\right): \delta$ 0.91-1.02 (m, 30H, $t$-butyl $+3 \times$ isopropyl), $2.99(\mathrm{~m}, 1 \mathrm{H}), 3.56\left(\mathrm{dd},{ }^{2} J 10 \mathrm{~Hz} \cdot{ }^{3} J 5.2 \mathrm{~Hz}\right.$, 1H), $4.02\left(\mathrm{dd},{ }^{2} J 10 \mathrm{~Hz},{ }^{3} J 5.2 \mathrm{~Hz}, 1 \mathrm{H}\right), 5.43\left(\mathrm{~d},{ }^{3} \mathrm{~J} 6.2 \mathrm{~Hz}\right.$, $1 \mathrm{H}, \mathrm{CH}), 5.95\left(\mathrm{~d},{ }^{2} \mathrm{~J} 1.1 \mathrm{~Hz}, 1 \mathrm{H}_{a}, \mathrm{CH}_{2}\right), 5.98\left(\mathrm{~d},{ }^{2} \mathrm{~J} 1.5 \mathrm{~Hz}\right.$, $\left.1 \mathrm{H}_{b}, \mathrm{CH}_{2}\right), 6.83$ (s, $1 \mathrm{H}$ aromatic), 6.9 (s, $1 \mathrm{H}$ aromatic), 7.33-7.6 (m, $10 \mathrm{H}$ aromatics). ${ }^{13} \mathrm{C}$ NMR $\left(75 \mathrm{MHz}, \mathrm{CDCl}_{3}\right)$ : $\delta 12.6,17.9,19.2,26.9,57.1,62.3,72,101.7,108.2,111.8$, 112.2, 127.5, 129.5, 133, 134.8, 135.4, 147.4, 147.7, 176.4. HRMS (EI, $70 \mathrm{eV}$ ) $\mathrm{m} / z$ Calc. for $\mathrm{C}_{36} \mathrm{H}_{49} \mathrm{BrO}_{6} \mathrm{Si}_{2}$ $[\mathrm{M}]^{+}:$712.2251. Found: 712.2262.

General procedure for the preparation of isoquinolinones 51, 52 and 53

To a solution of $(0.87 \mathrm{mmol})$ of the corresponding acids 48,49 and $\mathbf{5 0}$ in acetone $(8 \mathrm{~mL})$, cooled at $0{ }^{\circ} \mathrm{C}$, was added anydrous triethylamine (2.0 equiv.) and ethyl chloroformate (1.5 equiv.) and the resulting solution was stirred for $40 \mathrm{~min}$. After this time, an aqueous solution of $\mathrm{NaN}_{3}$ (1.5 equiv., $1.0 \mathrm{NaN}_{3}$ per $3 \mathrm{~mL}$ of distilled $\mathrm{H}_{2} \mathrm{O}$ ) was added and the mixture was stirred for $2 \mathrm{~h}$ at $0{ }^{\circ} \mathrm{C}$. Then the reaction mixture was diluted with dichloromethane $(10 \mathrm{~mL})$ and the organic layer was washed with brine $(5 \mathrm{~mL})$. The organic phase was dried over $\mathrm{Na}_{2} \mathrm{SO}_{4}$ and the solvent was evaporated. To the residue was added $15 \mathrm{~mL}$ of anhydrous toluene and the solution was refluxed for $2 \mathrm{~h}$ under an argon atmosphere. The reaction evolution was followed by IR. The disappearance of the absorption band at $2137 \mathrm{~cm}^{-1}$ attributed to the azide, accompanied by the appearance of an absorption band at $2260 \mathrm{~cm}^{-1}$, confirmed the formation of the isocyanate. After that, the solvent was evaporated and to the residue was added, under an argon atmosphere, anydrous ethyl ether $(10 \mathrm{~mL})$ and the reaction temperature was lowered to $-78^{\circ} \mathrm{C}$. Then, a solution of $s e c$-butyl lithium (3.0 equiv.) in ether was added. The resulting mixture was stirred for $2 \mathrm{~h}$ at $-78^{\circ} \mathrm{C}$. After that time, distilled water was slowly added and the reaction medium was extracted with ethyl ether $(3 \times 20 \mathrm{~mL})$. The combined organic phase was dried over 
anhydrous $\mathrm{Na}_{2} \mathrm{SO}_{4}$ and the solvent was evaporated. The residue was purified by flash silica gel column chromatography to furnished the corresponding isoquinolinones.

\section{(土)-8-(tert-Butyl-dimethyl-silanyloxy)-7-(tert-butyl-} diphenyl-silanyloxymethyl)-7,8-dihydro- $6 H[1,3]$ dioxol[4,5-g]-isoquinolin-5-one (51)

Colorless oil, $50 \%$ overall yield. IR $v_{\max } / \mathrm{cm}^{-1}: 3415$, 3070, 2956, 2930, 2857, 1670, 1612, 1264, 1112, 1038, 837, 701. ${ }^{1} \mathrm{H}-\mathrm{NMR}\left(500 \mathrm{MHz}, \mathrm{CDCl}_{3}\right): \delta-0.06(\mathrm{~s}, 3 \mathrm{H})$, $-0.01(\mathrm{~s}, 3 \mathrm{H}), 0.77(\mathrm{~s}, 9 \mathrm{H}), 1.02(\mathrm{~s}, 9 \mathrm{H}), 3.73(\mathrm{~m}, 1 \mathrm{H})$, $3.82\left(\mathrm{~d},{ }^{3} J 6.1 \mathrm{~Hz}, 2 \mathrm{H}\right), 4.82\left(\mathrm{~d},{ }^{3} J 4.3 \mathrm{~Hz}, 1 \mathrm{H}\right), 6.04$ (s, $2 \mathrm{H}, \mathrm{OCH}_{2} \mathrm{O}$ ), 6.77 (s, $1 \mathrm{H}$ aromatic), 7.15 (bs, $1 \mathrm{H}, \mathrm{NH}$ ), 7.36-7.64 (m, 10H, aromatics), 7.49 (s, $1 \mathrm{H}$ aromatic). ${ }^{13} \mathrm{C}-$ NMR $\left(125 \mathrm{MHz}, \mathrm{CDCl}_{3}\right): \delta-4.8,-4.6,18,19.1,25.6$, 26.7, 57.7, 62.6, 67, 101.9, 106.3, 108.1, 121.1, 127.9, $129.9,132.8,135.5,136.3,147.8,151.6,165.5$. HRMS (ESI) $m / z$ Calc. for $\mathrm{C}_{33} \mathrm{H}_{43} \mathrm{NNaO}_{5} \mathrm{Si}_{2}[\mathrm{M}+\mathrm{Na}]^{+}: 612.2572$. Found: 612. 2389.

(土)-7-(4-Methoxy-benzyloxymethyl)-8-triisopropylsilanyloxy-7,8-dihydro-6H-[1,3]dioxol-[4,5-g]-isoquinolin5-one (52)

Yellow-tinged oil, $50 \%$ overall yield. IR $v_{\max } / \mathrm{cm}^{-1}$ : 3371, 3011, 2956, 2931, 2858, 1670, 1612, 1513, 1474, 1250, 1111, 1038, 822, 755, 707. ${ }^{1} \mathrm{H}$ NMR (500 MHz, $\left.\mathrm{CDCl}_{3}\right)$ Diastereoisomers mixture: $\delta 0.96-1.05(\mathrm{~m}, 21 \mathrm{H}$, $3 \times$ isopropyl), 3.26-3.51 (m, $1 \mathrm{H}), 3.77(\mathrm{~m}, 2 \mathrm{H}), 3.80(\mathrm{~s}$, $3 \mathrm{H}), 4.32-4.45(\mathrm{~m}, 2 \mathrm{H}), 4.85\left(\mathrm{~d},{ }^{3} \mathrm{~J} 3.3 \mathrm{~Hz}, 1 \mathrm{H}\right.$ major diastereoisomer), $5.04\left(\mathrm{~d},{ }^{3} \mathrm{~J} 3.6 \mathrm{~Hz}, 1 \mathrm{H}\right.$, minor diastereoisomer), $6.02\left(\mathrm{~m}, 2 \mathrm{H}, \mathrm{CH}_{2}\right), 6.35(\mathrm{bs}, 1 \mathrm{H}, \mathrm{NH})$, 6.41 (bs, 1H, NH), 6.72 (s, 1H aromatic), 6.85-7.22 (m, $4 \mathrm{H}$ aromatics), 7.49 (s, $1 \mathrm{H}$ aromatic). ${ }^{13} \mathrm{C}$ NMR $(125 \mathrm{MHz}$, $\mathrm{CDCl}_{3}$ ) Major diastereoisomer: $\delta 12.7,17.9,55.2,56.5$, $68,68.7,73,101.6,107.7,108.2,113.8,122.4,129.5$, 129.6, 136, 147.9, 150.7, 159.3, 164.4. Minor diastereoisomers: $\delta 12.7,18,55.2,58,67.7,69.9,73.2,101.6,106$, $108,113.8,122.2,129.2,129.6,134.5,147.6,150.6,159.3$, 164.2. HRMS (ESI) $m / z$ Calc. for $\mathrm{C}_{28} \mathrm{H}_{39} \mathrm{NNaO}_{6} \mathrm{Si}[\mathrm{M}+$ $\mathrm{Na}^{+}:$536.2439. Found: 536.2412.

(土)-7-(tert-Butyl-diphenyl-silanyloxymethyl)-8-triisopropylsilanyloxy-7,8-dihydro-6H-[1,3]dioxol [4,5-g]isoquinolin-5-one (53)

Colorless oil, $50 \%$ overall yield. IR $v_{\max } / \mathrm{cm}^{-1}: 3204$, 3070, 2943, 2866, 1670, 1612, 1511, 1467, 1250, 1110, $1037,934,822,752 .{ }^{1} \mathrm{H}$ NMR $\left(300 \mathrm{MHz}, \mathrm{CDCl}_{3}\right): \delta 0.95-$ $1.15(\mathrm{~m}, 30 \mathrm{H}, t$-butyl $+3 \times$ isopropyl), 3.72-3.88 (m, $2 \mathrm{H})$, 4.21-4.24 (m, 1H), $5.0\left(\mathrm{~d},{ }^{3} J 2.9 \mathrm{~Hz}, 1 \mathrm{H}\right), 6.01(\mathrm{~s}, 2 \mathrm{H})$, 6.41 (bs, $1 \mathrm{H}, \mathrm{NH}), 6.83$ (s, $1 \mathrm{H}$ aromatic), $7.5(\mathrm{~s}, 1 \mathrm{H}$ aromatic), 7.33-7.64 (m, 10H aromatics). ${ }^{13} \mathrm{C}$ NMR (75 $\left.\mathrm{MHz}, \mathrm{CDCl}_{3}\right): \delta 12.7,18.1,19.1,26.8,58.2,62.9,67.9$, $101.7,106.2,108.2,121.9,127.8,129.8,132.9,135.5$, 136.2, 147.6, 151, 164.9. HRMS (EI, 70eV) $\mathrm{m} / \mathrm{z}$ Calc. for $\mathrm{C}_{36} \mathrm{H}_{49} \mathrm{NO}_{5} \mathrm{Si}_{2}[\mathrm{M}]^{+}:$631.3149. Found: $631.3100[\mathrm{M}]^{+}$.

\section{( \pm )-8-Hydroxy-7-hydroxymethyl-7,8-dihydro-6H-[1,3]} dioxol[4,5-g]-isoquinolin-5-one (11)

To a cooled solution of $\mathbf{5 1}(0.23 \mathrm{~g}, 0.4 \mathrm{mmol})$ in THF $(6 \mathrm{~mL})$, at $0{ }^{\circ} \mathrm{C}$, was added a $1.0 \mathrm{~mol} \mathrm{~L} \mathrm{~L}^{-1}$ solution of tetrabutyl ammonium fluoride (TBAF, $1.6 \mathrm{~mL}, 1.6 \mathrm{mmol}$ ) and the resulting solution was stirred at $0{ }^{\circ} \mathrm{C}$ for $5 \mathrm{~min}$, then the bath was removed and the reaction was stirred for $2 \mathrm{~h}$ at room temperature. After that, the solvent was removed under reduced pressure and the residue was diluted with ethyl acetate $(10 \mathrm{~mL})$. The organic layer was washed with a saturated solution of $\mathrm{NH}_{4} \mathrm{Cl}(2 \times 10 \mathrm{~mL})$, distilled water $(2 \times 10 \mathrm{~mL})$, brine $(2 \times 10 \mathrm{~mL})$ and dried over anhydrous sodium. After filtration, the solvent was removed and the residue was purified by flash silica gel column chromatography (ethyl acetate/methanol; 90:10) to provide 11 , as a white solid, in $76 \%$ yield.

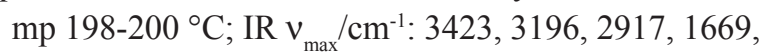
$1611,1503,1465,1408,1383,1262,1038,932,903,787$, 695, 490, 432. 'H NMR (300 MHz, DMSO- $\left.d_{6}\right)$ : diastereoisomers mixture, $\delta 3.34-3.70(\mathrm{~m}, 1 \mathrm{H}, \mathrm{CH}), 3.63(\mathrm{bs}, 2 \mathrm{H}$, $\left.\mathrm{CH}_{2}\right), 4.61\left(\mathrm{~d},{ }^{3} \mathrm{~J} 4.8 \mathrm{~Hz}, 1 \mathrm{H}\right), 4.78$ (bs, $\left.1 \mathrm{H}, \mathrm{OH}\right), 4.89$ (bs, $1 \mathrm{H}, \mathrm{OH}), 6.10$ (s, $\left.2 \mathrm{H}, \mathrm{CH}_{2} \mathrm{OCH}_{2}\right), 6.99$ (s, $1 \mathrm{H}$ aromatic), 7.29 (s, $1 \mathrm{H}$ aromatic), 7.48 (bs, $1 \mathrm{H}, \mathrm{NH}) \cdot{ }^{13} \mathrm{C}$ NMR (125 $\left.\mathrm{MHz}, \mathrm{DMSO}-d_{6}\right)$ : Major diastereoisomer, $\delta$ 59.8, 62.3, $65.7,102.4,107.1,107.9,122.8,137.3,147.7,150.9$, 163.6. Minor diastereoisomer, $\delta 57.8,60.8,65.4,102.4$, 107.2, 108.2, 123.7, 137.4, 147.9, 150.7, 164.3. HRMS (EI, $70 \mathrm{eV}$ ) $\mathrm{m} / z$ Calc. for $\mathrm{C}_{11} \mathrm{H}_{11} \mathrm{NO}_{5}\left[\mathrm{M}^{+}\right]$: 237.0637 . Found: 237.0622 .

(土)-2-(6-Bromo-benzo[1,3]dioxol-5-yl)-2-(tert-butyldimethyl-silanyloxy)-1-(tert-butyl-diphenyl-silanyloxymethyl)-ethylamine (62)

To a solution of acid $48(1.0 \mathrm{~g}, 1.48 \mathrm{mmol})$ in acetone $(10 \mathrm{~mL})$, cooled at $0{ }^{\circ} \mathrm{C}$, was added anhydrous triethylamine (0.41 mL, $2.96 \mathrm{mmol}, 2.0$ equiv.) and freshly distilled ethyl chloroformate $(0.22 \mathrm{~mL}, 2.2 \mathrm{mmol}, 1.5$ equiv.). The resulting solution was stirred for $40 \mathrm{~min}$ at $0{ }^{\circ} \mathrm{C}$. After this time, an aqueous solution of $\mathrm{NaN}_{3}(0.43$ $\mathrm{mL}, 1.0 \mathrm{~g} \mathrm{NaN}_{3}$ per $3 \mathrm{~mL}$ of distilled $\mathrm{H}_{2} \mathrm{O}$ ) was added. The mixture was stirred for $1 \mathrm{~h}$. The mixture was diluted with dichloromethane $(10 \mathrm{~mL})$ and, after separation, the organic phase was successively washed with distilled water $(10 \mathrm{~mL})$ and brine $(10 \mathrm{~mL})$. The organic phase was then dried over $\mathrm{Na}_{2} \mathrm{SO}_{4}$ and the solvent was evaporated. To the 
residue was added anydrous toluene $(15 \mathrm{~mL})$ and the resulting solution was refluxed for $2 \mathrm{~h}$ under an argon atmosphere. The evolution of the reaction was followed by IR. The disappearance of the absorption band at 2137 $\mathrm{cm}^{-1}$, atributted to the acylazide associated with the appearance of the absorption band at $2255 \mathrm{~cm}^{-1}$, confirmed isocyanate formation. The solvent was then evaporated and the residue was dissolved in THF $(10 \mathrm{~mL})$. The solution was cooled to $0{ }^{\circ} \mathrm{C}$ and a solution of $\mathrm{NaOH}(1.5$ $\mathrm{mL}, 1.5 \mathrm{~mol} \mathrm{~L}^{-1}$ ) was slowly added. After that the resulting solution was stirred for $4 \mathrm{~h}$ at $70{ }^{\circ} \mathrm{C}$. After cooling to the room temperature, the reaction medium was diluted with ethyl ether $(30 \mathrm{~mL})$ and distilled water $(10 \mathrm{~mL})$. The phases were separated and the organic one was dried over $\mathrm{Na}_{2} \mathrm{SO}_{4}$ and the solvent was evaporated. The residue was purified by flash silica gel column chromatography (hexane/ethyl acetate $50 \%$ ) to furnish 62 , as a colorless oil, in $62 \%$ overall yield.

IR $v_{\max } / \mathrm{cm}^{-1}: 3375,2953,2929,2856,1474,1252$, 1237, 1112, 1040, 837, 778, 702. 'H NMR (300 MHz, $\left.\mathrm{CDCl}_{3}\right): \delta-0.13(\mathrm{~s}, 3 \mathrm{H}) ; 0.07(\mathrm{~s}, 3 \mathrm{H}), 0.90(\mathrm{~s}, 9 \mathrm{H}), 1.10$ (s, 9H), 2.76 (bs, $\left.2 \mathrm{H}, \mathrm{NH}_{2}\right), 2.98(\mathrm{~m}, 1 \mathrm{H}), 3.63$ (d, ${ }^{3} \mathrm{~J} 6.6$ $\mathrm{Hz}, 2 \mathrm{H}), 5.21\left(\mathrm{~d},{ }^{3} J 2.9 \mathrm{~Hz}, 1 \mathrm{H}\right), 5.93\left(\mathrm{~d},{ }^{2} J 1.5 \mathrm{~Hz}, 1 \mathrm{H}_{a}\right.$, $\left.\mathrm{CH}_{2}\right), 5.99\left(\mathrm{~d},{ }^{2} J 1.5 \mathrm{~Hz}, 1 \mathrm{H}_{b}, \mathrm{CH}_{2}\right), 6.94$ (s, $1 \mathrm{H}$ aromatic), 6.98 (s, $1 \mathrm{H}$ aromatic), 7.35-7.7 (m, $10 \mathrm{H}$ aromatics). ${ }^{13} \mathrm{C}-$ $\operatorname{NMR}\left(75 \mathrm{MHz}, \mathrm{CDCl}_{3}\right): \delta-4.9,-4.4,18.2,19.3,26,27.1$, 57.8, 65.6, 72.3, 101.5, 108.9, 111.5, 112.1, 127.5, 129.5, 133.3, 134.8, 135.4, 135.5, 147, 147.3. HRMS (ESI) $\mathrm{m} / \mathrm{z}$ Calc. for $\mathrm{C}_{32} \mathrm{H}_{44} \mathrm{BrNO}_{4} \mathrm{Si}_{2}[\mathrm{M}+2]^{+}$- $t$-butyl: 586.1268 . Found: 586.1203.

(土)-[2-(6-Bromo-benzo[1,3]dioxol-5-yl)-2-(tert-butyldimethyl-silanyloxy)-1-(tert-butyl-diphenyl-silanyloxymethyl)-ethyl]-[2-(4-methoxy-phenyl)-ethyl]amine (63)

A mixture of amine 59 (0.62 g, $0.4 \mathrm{mmol}), \mathrm{Na}_{2} \mathrm{CO}_{3}$ (0.13 g, $1.2 \mathrm{mmol}$ ) and 2-bromoethyl-(4-methoxy)benzene $(0.24 \mathrm{~g}, 0.6 \mathrm{mmol})$ in anhydrous acetonitrile $(15 \mathrm{~mL})$, under an argon atmosphere, was refluxed for $24 \mathrm{~h}$. After that time, the solvent was evaporated and to the residue was added distilled water $(15 \mathrm{~mL})$. The aqueous phase was extracted with dichloromethane $(30 \mathrm{~mL})$ and the organic phase was dried over anhydrous $\mathrm{Na}_{2} \mathrm{SO}_{4}$ and the solvent was evaporated. The residue was purified by flash silica gel column chromatography (hexane/ethyl acetate 20\%) to afford alkylated amine $\mathbf{6 3}$ as a yellow-tinged oil in $85 \%$ yield.

IR $v_{\max } / \mathrm{cm}^{-1}: 3432,2952,2929,2856,1735,1611$, 1512, 1474, 1274, 1111, 1077, 1039, 937, 837, 702. ${ }^{1} \mathrm{H}$ NMR (500 MHz, $\left.\mathrm{CDCl}_{3}\right): \delta-0.15(\mathrm{~s}, 3 \mathrm{H}), 0.08(\mathrm{~s}, 3 \mathrm{H})$, 0.87 (s, 9H), 1.09 (s, 9H), $2.29(\mathrm{~m}, 1 \mathrm{H}), 2.45(\mathrm{~m}, 2 \mathrm{H})$, $2.61(\mathrm{~m}, 1 \mathrm{H}), 2.8(\mathrm{~m}, 1 \mathrm{H}), 3.65(\mathrm{~m}, 2 \mathrm{H}), 3.80(\mathrm{~s}, 3 \mathrm{H})$, $5.30\left(\mathrm{~d},{ }^{3} J 5.2 \mathrm{~Hz}, 1 \mathrm{H}\right), 5.98\left(\mathrm{~d},{ }^{2} J 1.5 \mathrm{~Hz}, 1 \mathrm{H}\right), 6.01\left(\mathrm{~d},{ }^{2} \mathrm{~J}\right.$ $1.5 \mathrm{~Hz}, 1 \mathrm{H}), 6.79(\mathrm{~s}, 1 \mathrm{H}), 6.80\left(\mathrm{~d},{ }^{3} J 8.2 \mathrm{~Hz}, 2 \mathrm{H}\right), 6.94(\mathrm{~d}$, $\left.{ }^{3} J 8.2 \mathrm{~Hz}, 2 \mathrm{H}\right), 7.40(\mathrm{~s}, 1 \mathrm{H}, \mathrm{CH}), 7.36-7.66(\mathrm{~m}, 10 \mathrm{H}$, aromatics). ${ }^{13} \mathrm{C}$ NMR $\left(125 \mathrm{MHz}, \mathrm{CDCl}_{3}\right): \delta-5.9$, $-4.9,18,19.3,25.4,26.8,34.6,50.8,54.7,61.6,64.3$, $65.8,101.9,108.2,109.8,113.4,127.5,129.4,129.7,130$, $131.9,132,132.7,132.9,135.4,135.5,136.3,146.1$, 146.6, 147, 158. HRMS (ESI) $\mathrm{m} / \mathrm{z}$ Calc. for $\mathrm{C}_{41} \mathrm{H}_{54} \mathrm{NNaBrO}_{5} \mathrm{Si}_{2}[\mathrm{M}+\mathrm{Na}]^{+}:$798.2616. Found: 798.2601 .

(土)-[2-(6-Bromo-benzo[1,3]dioxol-5-yl)-2-(tert-butyldimethyl-silanyloxy)-1-(tert-butyl-diphenyl-silanyloxymethyl)-ethyl]-[2-(4-methoxy-phenyl)-ethyl]-methoxycarbamate (64)

To a solution of amine $\mathbf{6 3}(0.26 \mathrm{~g}, 0.2 \mathrm{mmol})$ in dry acetone $(10 \mathrm{~mL})$, cooled to $0{ }^{\circ} \mathrm{C}$, was added anhydrous triethylamine $(56 \mathrm{~mL}, 0.4 \mathrm{mmol})$ and methyl chloroformate $(46 \mathrm{~mL}, 0.6 \mathrm{mmol})$. The resulting solution was stirred for $40 \mathrm{~min}$ at $0{ }^{\circ} \mathrm{C}$. After this time, the solvent was evaporated and the residue was diluted with ethyl acetate $(20 \mathrm{~mL})$. The organic phase was successively washed with distilled water $(2 \times 20 \mathrm{~mL})$ and brine $(2 \times 20 \mathrm{~mL})$ and finally was dried over anhydrous $\mathrm{Na}_{2} \mathrm{SO}_{4}$. The solvent was evaporated and the residue was purified by flash silica gel column chromatography (hexane/ethyl acetate $20 \%$ ) to give methoxy carbamate 64 as a colorless oil in $90 \%$ yield.

IR $v_{\max } / \mathrm{cm}^{-1}: 3012,2998,2945,2929,2891,2864$, 1704, 1699, 1608, 1510, 1476, 1471, 1464, 1259, 1238, 1073, 1039, 936, 837, 701. 'H NMR (300 MHz, $\left.\mathrm{CDCl}_{3}\right)$ : $\delta-0.24(\mathrm{~s}, 3 \mathrm{H}),-0.01(\mathrm{~s}, 3 \mathrm{H}), 0.82(\mathrm{~s}, 9 \mathrm{H}), 1.08(\mathrm{~s}, 9 \mathrm{H})$, $3.45(\mathrm{~s}, 3 \mathrm{H}), 3.8(\mathrm{~s}, 3 \mathrm{H}), 3.67\left(\mathrm{~d},{ }^{2} J 11 \mathrm{~Hz}, 2 \mathrm{H}\right), 5.08\left(\mathrm{~d},{ }^{3} \mathrm{~J}\right.$ $4.4 \mathrm{~Hz}, 1 \mathrm{H}), 5.95\left(\mathrm{~d},{ }^{2} J 1.5 \mathrm{~Hz}, 1 \mathrm{H}\right), 5.98\left(\mathrm{~d},{ }^{2} J 1.5 \mathrm{~Hz}\right.$, 1H), $6.8(\mathrm{~s}, 1 \mathrm{H}), 6.83\left(\mathrm{~d},{ }^{3} J 8.4 \mathrm{~Hz}, 2 \mathrm{H}\right), 6.84(\mathrm{~s}, 1 \mathrm{H}), 7.11$ (d, $\left.{ }^{3} \mathrm{~J} 8.4 \mathrm{~Hz}, 2 \mathrm{H}\right), 7.34-7.7$ (m, 10H aromatics). ${ }^{13} \mathrm{C}$ NMR $\left(75 \mathrm{MHz}, \mathrm{CDCl}_{3}\right): \delta-5.4,-4.8,17.8,19.2,25.6,26.8$, 34.7, 46.9, 52, 53.9, 61.1, 61.8, 73.8, 101.9, 108.4, 111.7, $111.8,119.8,127.7,129.6,129.7,132.4,133.2,134.2$, 135.6, 147.2, 147.3, 156.2, 157.1. HRMS (ESI) $\mathrm{m} / \mathrm{z}$ Calc. for $\mathrm{C}_{43} \mathrm{H}_{56} \mathrm{BrNNaO}_{7} \mathrm{Si}_{2}[\mathrm{M}+\mathrm{Na}]^{+}:$856.2671. Found: 856.2589 .

( \pm )-8-(tert-Butyl-dimethyl-silanyloxy)-7-(tert-butyldiphenyl-silanyloxymethyl)-6-[2-(4-methoxy-phenyl)ethyl]-7,8-dihydro-6H-[1,3]dioxol[4,5-g]-isoquinolin-5one (65)

To a solution of carbamate $64(0.15 \mathrm{~g}, 0.18 \mathrm{mmol})$ in anhydrous ethyl ether $(5 \mathrm{~mL})$, under an orgon atmosphere and at $-78{ }^{\circ} \mathrm{C}$, was slowly added a solution $0.8 \mathrm{~mol} \mathrm{~L}^{-1}$ of tert-butyl lithium $(0.7 \mathrm{~mL}, 0.54 \mathrm{mmol})$. The resulting 
solution was stirred for $1 \mathrm{~h}$ at $-78^{\circ} \mathrm{C}$. After this time, the reaction medium was diluted with ethyl ether $(15 \mathrm{~mL})$ and distilled water $(10 \mathrm{~mL})$. The phases were separated and the organic one was dried over anhydrous $\mathrm{Na}_{2} \mathrm{SO}_{4}$. The solvent was evaporated and the residue was purified by flash silica gel column chromatography (hexane/ethyl acetate, $25 \%$ ) to give isoquinolinone $\mathbf{6 5}$ as a colorless oil in $60 \%$ yield.

IR $v_{\max } / \mathrm{cm}^{-1}: 3069,2952,2929,2893,2856,1654$, $1611,1512,1467,1416,1272,1248,1177,1111,1038$, 937, 839, 823, 702. ${ }^{1} \mathrm{H}$ NMR (300 $\left.\mathrm{MHz}, \mathrm{CDCl}_{3}\right): \delta-0.17$ (s, 3H), 0.03 (s, 3H), 0.85 (s, 9H), 0.92 (s, 9H), 2.88 (m, $\left.1 \mathrm{H}_{a}, \mathrm{CH}_{2}\right), 3.05-3.18\left(\mathrm{~m}, 2 \mathrm{H}, \mathrm{CH}_{2}\right), 3.26\left(\mathrm{~m}, 1 \mathrm{H}_{b}, \mathrm{CH}_{2}\right)$, 3.61-3.76 (m, 2H), 3.79 (s, 3H), 4.61-4.68 (m, 1H), 4.77 (d, $\left.{ }^{3} J 6.2 \mathrm{~Hz}, 1 \mathrm{H}\right), 5.94$ (d, $\left.{ }^{2} J 1.3 \mathrm{~Hz}, 1 \mathrm{H}\right), 5.97$ (d, ${ }^{2} J 1.3$ $\mathrm{Hz}, 1 \mathrm{H}), 6.73$ (s, 1H), 6.85 (d, $\left.{ }^{3} J 8.8 \mathrm{~Hz}, 2 \mathrm{H}\right), 7.16\left(\mathrm{~d},{ }^{3} \mathrm{~J}\right.$ $8.8 \mathrm{~Hz}, 2 \mathrm{H}), 7.49$ (s, $1 \mathrm{H}), 7.30-7.57(\mathrm{~m}, 10 \mathrm{H}$ aromatics $) .{ }^{13} \mathrm{C}$ NMR (75 MHz, $\left.\mathrm{CDCl}_{3}\right): \delta-5.6,-4.9,17.9,18.9,25.7$, 26.5, 33.6, 51, 55.1, 61.8, 64.1, 67.5, 101.3, 103.9, 107.9, $113.9,121.7,127.7,129.6,129.7,130,131.6,132.7,132.9$, 135.4, 135.5, 136.3, 146.8, 150.6, 158.1, 163.1. HRMS (ESI) $m / z$ Calc. for $\mathrm{C}_{42} \mathrm{H}_{53} \mathrm{NNaO}_{6} \mathrm{Si}_{2}[\mathrm{M}+\mathrm{Na}]^{+}: 746.3304$. Found: 746.3295 .

( \pm )-8-Hydroxy-7-hydroxymethyl-6-[2-(4-methoxy-phenyl)ethyl]-7,8-dihydro-6H-[1,3]dioxol[4,5-g]-isoquinolin-5one (70)

To a solution of isoquinolinone $\mathbf{6 5}(0.04 \mathrm{~g}, 0.05 \mathrm{mmol})$ in THF $(5 \mathrm{~mL})$ at $0{ }^{\circ} \mathrm{C}$ was added a solution of tetrabutyl ammonium fluoride (TBAF) in THF $(0.25 \mathrm{~mL}, 0.25$ $\mathrm{mmol}$ ). After $5 \mathrm{~min}$, the ice bath was removed and the reaction temperature was allow to warm to the room temperature. Finally the reaction was stirred for $1 \mathrm{~h}$ at this temperature. Then, the solvent was evaporated and the residue was dissolved in ethyl acetate. The organic phase was successively washed with a saturated solution of $\mathrm{NH}_{4} \mathrm{Cl}$, distilled water $(2 \times 20 \mathrm{~mL})$ and finally dried over anhydrous $\mathrm{Na}_{2} \mathrm{SO}_{4}$. The solvent was evaporated and the residue was purified by flash silica gel column chromatography (hexane/ethyl acetate/methanol, 50:40:10) to give isoquinolinone 70, as yellow-tinged solid, in $75 \%$ yield.

mp 96.1-96.7 ${ }^{\circ} \mathrm{C} . I_{\mathrm{IR}} v_{\max } / \mathrm{cm}^{-1}: 3418,3070,2957,2929$, 2888, 2856, 1592, 1471, 1463, 1427, 1362, 1114, 1001, 820, 739, 701. ${ }^{1} \mathrm{H} \mathrm{NMR}\left(300 \mathrm{MHz}, \mathrm{CDCl}_{3}\right): \delta 2.0(\mathrm{bs}, 1 \mathrm{H}$, $\mathrm{OH}), 2.93(\mathrm{~m}, 1 \mathrm{H}), 3.02-3.08(\mathrm{~m}, 2 \mathrm{H}), 3.50(\mathrm{~m}, 1 \mathrm{H}), 3.68$ $(\mathrm{m}, 1 \mathrm{H}), 3.80(\mathrm{~s}, 3 \mathrm{H}), 3.86(\mathrm{~m}, 1 \mathrm{H}), 4.32-4.40(\mathrm{~m}, 1 \mathrm{H})$, $5.04\left(\mathrm{~d},{ }^{3} \mathrm{~J} 5.5 \mathrm{~Hz}, 1 \mathrm{H}\right), 6.02(\mathrm{~s}, 2 \mathrm{H}), 6.85\left(\mathrm{~d},{ }^{2} J 8.5 \mathrm{~Hz}\right.$, 2H), 7.04 (s, 1H), 7.17 (d, $\left.{ }^{3} J 8.5 \mathrm{~Hz}, 2 \mathrm{H}\right), 7.44$ (s, 1H). ${ }^{13} \mathrm{C} \mathrm{NMR}\left(125 \mathrm{MHz}, \mathrm{CDCl}_{3}\right): \delta 34.1,50.9,55.4,62.5$, 64.3, 67.8, 101.7, 104.3, 109.7, 115.1, 115.9, 122.2, 129.9,
130.2, 132.6, 136.2, 147, 151.2, 158.3, 163.5. HRMS (70 eV, EI) Calc. for $\mathrm{C}_{20} \mathrm{H}_{21} \mathrm{NO}_{6}$ 371.1369. Found: 371.1360.

( \pm )-7-(tert-Butyl-diphenyl-silanyloxymethyl)-8-hydroxy-6-[2(4-methoxy-phenyl)-ethyl]-7,8-dihydro-6H-[1,3]dioxol[4,5g]-isoquinolin-5-one (71)

A mixture of dihydroisoquinoline 70 (0.025 g, 0.067 mmol), a crystal of DMAP, anhydrous triethylamine $(100 \mu \mathrm{L}, 0.1 \mathrm{mmol})$ and tert-butyldiphenylsilyl chloride $(20 \mu \mathrm{L}, 0.013 \mathrm{mmol})$ in dry dichloromethane was stirred for $24 \mathrm{~h}$ at room temperature. After this period of time, the mixture was diluted with dichloromethane $(10 \mathrm{~mL})$ and the organic phase was washed with a saturated solution of $\mathrm{NaHCO}_{3}(2 \times 10 \mathrm{~mL})$. The phases were separated and the organic one was dried over anhydrous magnesium sulfate. The solvent was evaporated and the residue was purified by flash silica gel column chromatography (hexane/ethyl acetate 20\%) to give mono-protected isoquinolinone $\mathbf{7 0}$, as a colorless oil, in $80 \%$ yield.

IR $v_{\max } / \mathrm{cm}^{-1}: 3336,3009,2949,2930,2853,1650$, $1634,1602,1513,1471,1427,1272,1248,1112,1076$, 1038, 823, 756, 703. ${ }^{1} \mathrm{H}$ NMR (300 $\left.\mathrm{MHz}, \mathrm{CDCl}_{3}\right)$ : $\delta 1.02(\mathrm{~s}, 9 \mathrm{H}), 2.63-2.82(\mathrm{~m}, 2 \mathrm{H}), 3.50(\mathrm{~m}, 1 \mathrm{H}), 3.63(\mathrm{~m}$, $1 \mathrm{H}), 3.61-3.76(\mathrm{~m}, 2 \mathrm{H}) ; 3.80(\mathrm{~s}, 3 \mathrm{H}) ; 4.12-4.20(\mathrm{~m}, 1 \mathrm{H})$; $5.01\left(\mathrm{~d},{ }^{3} J 5.5 \mathrm{~Hz}, 1 \mathrm{H}\right), 6.03\left(\mathrm{~d},{ }^{2} J 1.1 \mathrm{~Hz}, 1 \mathrm{H}\right), 6.06\left(\mathrm{~d},{ }^{2} J\right.$ $1.1 \mathrm{~Hz}, 1 \mathrm{H}), 6.85$ (d, ${ }^{3} J 8.8 \mathrm{~Hz}, 2 \mathrm{H}$ aromatics), $7.07\left(\mathrm{~d},{ }^{3} \mathrm{~J}\right.$ $8.8 \mathrm{~Hz}, 2 \mathrm{H}$ aromatics), $7.15(\mathrm{~s}, 1 \mathrm{H}), 7.40(\mathrm{~s}, 1 \mathrm{H}), 7.31-$ $7.62\left(\mathrm{~m}, 10 \mathrm{H}\right.$ aromatics). ${ }^{13} \mathrm{C}$ NMR $\left(75 \mathrm{MHz}, \mathrm{CDCl}_{3}\right)$ : $\delta 18.8,26.8,34,48.9,55.3,59.8,63.3,68.6,101.8,104.7$, $108,114,121.7,127.8,127.5,130.2,130.4,131.6,131.8$, 132.1, 135.2, 135.5, 136.3, 146.9, 150.4, 158.3, 163.2. HRMS (70eV, EI) Calc. for $\mathrm{C}_{36} \mathrm{H}_{39} \mathrm{NO}_{6} \mathrm{Si}\left[\mathrm{M}^{+}-t\right.$-Bu $]$ 550.2547. Found: 550.2539 .

\section{Acknowledgments}

The authors thank FAPESP for grants to ECSL (\# 00/ 07938-4) and for financial support (98/14640-0 and 02/ 00461-3). The authors also thank CNPq for a research fellowship to FC (301369/87-9). We thank Prof. C.H. Collins for English revision of this text.

\section{References}

1. Pettit, G. R.; Gaddamidi, V.; Herald, D. L.; Singh, S. B.; Cragg, G. M.; Schmidt, J. M.; Boettner, F. E.; Williams, M.; Sagawa, Y.; J. Nat. Prod. 1986, 49, 995; Pettit, G. R.; Gaddamidi, V.; Cragg, G. M.; J. Nat. Prod. 1984, 47, 1018; Pettit, G. R.; Pettit III, G. R.; Bachaus, R. A.; Boyd, M. R.; Meerow, A. W.; J. Nat. Prod. 1993, 56, 1682. 
2. Hoshino, O. In The Alkaloids; Cordell, G. A., ed.; AC Press: San Diego, 1998, vol. 51, pp. 323-424; Martin, S. F. In The Alkaloids; Brossi, A., ed.; AC Press: San Diego, 1987, vol. 39, pp. 251-376.

3. For a discusssion of the biological effects of galanthamine, see: Pearson, V. E.; Ann. Pharmacother. 2001, 35, 1406; for the total synthesis of Galanthamine, see: Trost, B. M.; Chem. Pharm. Bull. 2002, 50, 1 and references cited therein; for a recent review concerning the synthesis of galanthamine, see: Marco-Contelles, J.; Rodriguez, C.; Garcia, A. G.; Expert Opin. Ther. Pat. 2005, 15, 575 (CAS 2005, 143, 440594); Marco, L.; Carreiras, M. D. C.; Recent Pat. CNS Drug Discovery 2006, 1, 105 (CAS 2006, 144, 266426).

4. Pettit, G. R.; Gaddamidi, V.; Cragg, G. M.; Herald, D. L.; Sagawa, Y.; J. Chem. Soc. Chem. Commun. 1984, 1693; for a recent review concerning the synthesis of pancratistatin, see: Chapleur, Y.; Chretien, F.; Ibn Ahmed, S.; Khaldi, M.; Curr. Org. Synth. 2006, 3, 341; McNulty, J.; Larichev, V.; Pandey, S.; Bioorg. Med. Chem. Lett. 2005, 15, 5315; Rinner, U.; Hillebrenner, H. L.; Adams, D. R.; Hudlicky, T.; Pettit, G. R.; Bioorg. Med. Chem. Lett. 2004, 14, 2911; Kireev, A. S.; Nadein, O. N.; Agustin, V. J.; Bush, N. E.; Evidente, A.; Manpadi, M.; Ogasawara, M. A.; Rastogi, S. K.; Rogelj, S.; Shors, S. T.; Kornienko, A.; J. Org. Chem. 2006, 71, 5694.

5. Okamoto, T.; Torii, Y.; Isogai, Y.; Chem. Pharm. Bull. 1968, 16, 1860; for the biosynthesis of narciclasine, see: Fuganti, C.; Mazza, M.; J. Chem. Soc. Chem. Commun. 1972, 239 and references cited therein; Pettit, G. R.; Melody, N.; Herald, D. L.; Knight, J. C.; Chapuis, J.-C.; J. Nat. Prod. 2007, 70, 417.

6. Chattopadhyay, S.; Chattopadhyay, U.; Mathur, P. P.; Saini, K. S.; Ghosal, S.; Planta Med. 1983, 49, 252; for hippadine synthesis, see: Harrowven, D. C.; Lai, D.; Lucas, M. C.; Synthesis 1999, 1300; for recent examples concerning the synthesis of hippadine, see: Mentzel, U. V.; Tanner, D.; Tønder, J. E.; J. Org. Chem. 2006, 71, 5807; Ganton, M. D.; Kerr, M. A.; Org. Lett. 2005, 7, 4777.

7. Hansel, R.; Thober, H.; Arch. Pharm. 1982, 315, 767; for the total synthesis of anhydrolycorinone see, Boger, D. L.; Wolkenberg, S. E.; J. Org. Chem. 2000, 65, 9120 and references cited therein; Knoelker, H.-J.; Filali, S.; Synlett 2003, 1752; Wolkenberg, S. E.; Boger, D. L.; J. Org. Chem. 2002, 67, 7361; Harayama, T.; Hori, A.; Abe, H.; Takeuchi, Y.; Heterocycles 2003, 60, 2429.

8. For the isolation of (+)-Plicamine, see: Ünver, N.; Gözler, T.; Walch, N.; Gözler, B.; Hesse, M.; Phytochemistry 1999, 50, 1255.

9. For biological activity of pancratistatin and other alkaloids structurally related to it, see: Mutsuga, M.; Kojima, K.; Yamashita, M.; Ohno, T.; Ogihara, Y.; Inoue, M.; Biol. Pharm. Bull. 2002, 25, 223; Ouarzane-Amara, M.; Franetich, J. F.; Pettit, G. R.; Meijer, L.; Doerig, C.; Desportes-Livage, I.; Antimicrob.
Agents Chemother. 2001, 45, 3409; Pettit, G. R.; Orr, B.; Ducki, S.; Anti-Cancer Drug Des. 2000, 15, 389.

10. Jimenez, A.; Sanchez, L.; Vazquez, D.; FEBS Lett. 1975, 55, 53; Jimenez, A.; Santos, A.; Alonso, G.; Vazquez, D.; Biochim. Biophys. Acta 1976, 425, 342.

11. Eight total syntheses of pancratistatin have been reported to date: Danishefsky, S.; Lee, J. Y.; J. Am. Chem. Soc. 1989, 111, 4829; Tian, X.; Koenigsberger, K.; Hudlicky, T.; J. Am. Chem. Soc. 1995, 117, 3643; Hudlicky, T.; Tian, X.; Koenigsberger, K.; Maurya, R.; Rouden, J.; Fan, B.; J. Am. Chem. Soc. 1996, 118, 10752; Trost, B. M.; Pulley, S. R.; J. Am. Chem. Soc. 1995, 117, 10143; Magnus, P.; Sebhat, I. K.; J. Am. Chem. Soc. 1998, 120, 5341; Rigby, J. H.; Maharoof, U. S. M.; Mateo, M. E.; J. Am. Chem. Soc. 2000, 122, 6624; Doyle, T. J.; Hendrix, M.; VanDerveer, D.; Javanmard, S.; Haseltine, J.; Tetrahedron 1997, 53, 11153; Pettit, G. R.; Melody, N.; Herald, D. L.; J. Org. Chem. 2001, 66, 2583; Kim, S.; Ko, H. J.; Kim, E.; Kim, D.; Org. Lett. 2002, 4, 1343; Ko, H. J.; Kim, E.; Park, J. E.; Kim, D.; Kim, S.; J. Org. Chem. 2004, 69, 112; for the total synthesis of narciclasine, see: Rigby, J. H.; Mateo, M. E.; J. Am. Chem. Soc. 1997, 119, 12655; for the synthesis of the pancratistatin derivative, see: McNulty, J.; Mao, J.; Gibe, R.; Mo, R.; Wolf, S.; Pettit, G. R.; Herald, D. L.; Boyd, M. R.; Bioorg. Med. Chem. Lett. 2001, 11, 169 and references cited therein.

12. Tian, X. R.; Hudlicky, T.; Konigsberger, K.; J. Am. Chem. Soc. 1995, 117, 3643.

13. Mateus, C. R.; Feltrin, M. P.; Costa, A. M.; Almeida, W. P.; Coelho, F.; Tetrahedron 2001, 57, 6901; Masunare, A.; Ishida, E.; Trazzi, G.; Almeida, W. P.; Coelho, F.; Synth. Commun. 2001, 31, 2127; Coelho, F.; Rossi, R. C.; Tetrahedron Lett. 2002, 43, 2797; Mateus, C. R.; Coelho, F.; J. Braz. Chem. Soc. 2005, 16, 386; Silveira, G. P. C.; Coelho, F.; Tetrahedron Lett. 2005, 46, 6477; Mateus, C. R.; Coelho, F.; J. Braz. Chem. Soc. 2006, 17, 427.

14. For a nice and elegant first total synthesis of (+)-Plicamine, see: Baxendale, I. R.; Ley, S. V.; Piutti, C.; Angew. Chem., Int. Ed. 2002, 41, 2194; Baxendale, I. R.; Ley, S. V.; Nessi, M.; Piutti, C.; Tetrahedron 2002, 58, 6285.

15. Martin, S. F.; Davidsen, S. K.; J. Am. Chem. Soc. 1984, 106, 6431; Martin, S. F.; Davidsen, S. K.; Puckette, T. A.; J. Org. Chem. 1987, 52, 1962 and references cited therein.

16. Kane, V. V.; Synth. Commun. 1976, 3, 237; Kane, V. V.; Maitland, J. J.; Org. Synth. 1990, 7, 473.

17. For reviews see: Basavaiah, D.; Rao, P. D.; Hyma, R. S.; Tetrahedron 1996, 52, 8001; Ciganek, E. In Organic Reactions; John Wiley \& Sons Inc.: New York, 1997, vol. 51, Ch. 2, p. 201; Almeida, W. P.; Coelho, F.; Quim. Nova 2000, 23, 98; Basavaiah, D.; Rao, A. J.; Satyanarayana, T.; Chem. Rev. 2003, 103, 811; Langer, P.; Angew. Chem., Int. Ed. 2000, 39,3049 . 
18. For a recent discussion concerning new insights about the Morita-Baylis-Hillman reaction mechanism, see: Santos, L. S.; Pavam, C. H.; Almeida, W. P.; Coelho, F.; Eberlin, M. N.; Angew. Chem., Int. Ed. 2004, 43, 4330; Aggarwal, V. K.; Fulford, S. Y.; Lloyd-Jones, G. C.; Angew. Chem., Int. Ed. 2005, 44, 1706; Price, K. E.; Broadwater, S. J.; Walker, B. J.; McQuade, D. T.; J. Org. Chem. 2005, 70, 3980; Price, K. E.; Broadwater, S. J.; Jung, H. M.; McQuade, D. T.; Org. Lett. 2005, 7, 147. Amarante, G. W.; Benassi, M.; Sabino, A. A.; Esteves, P. M.; Coelho, F.; Eberlin, M. N.; Tetrahedron Lett. 2006, 47, 8427.

19. Almeida, W. P.; Coelho F.; Tetrahedron Lett. 1998, 39, 8609; Coelho, F.; Almeida, W. P.; Veronese, D.; Lopes, E. C. S.; Silveira, G. P. C; Rossi, R. C.; Pavam, C. H.; Tetrahedron 2002, 58, 7437.

20. Ley, S. V.; Norman, J.; Griffith, W. P.; Marsden, S. P.; Synthesis 1994, 639.

21. Bal, B. S.; Childers, W. E.; Pinnick, H. W.; Tetrahedron 1981, 37, 2091; Dalcanale, E.; Montanari, F.; J. Org. Chem. 1986, $51,567$.

22. Braibante, M. E. F.; Braibante, H. S.; Costenaro, E. R.; Synthesis 1999, 943; Sibi, M. P.; Lu, J.; Edwards, J.; J. Org. Chem. 1997, 62, 5864; Smith, P. A. S.; Org. React. 1946, 3, 337.

23. Coelho, F.; Lopes, E. C. S.; Veronese, D.; Rossi, R. C.; Tetrahedron Lett. 2003, 44, 5731.

24. Magnus, P.; Sebhat, I.; Tetrahedron 1998, 54, 15509.

25. Whaley, M.; Govindachari, T. R.; Org. React. 1951, 6, 74; Itoh, N.; Sugasawa, S.; Tetrahedron 1959, 6, 16; Fodor, G.; Nagubandi, S.; Tetrahedron 1980, 36, 1279; Banwell, M. G.; Cowden, C. J.; Mackay, M. F.; Chem. Commun. 1994, 61; Banwell, M. G.; Bissett, B. D.; Busato, S; Cowden, C. J.;
Hockless, D. C. R.; Holman, J. W.; Read, R. W.; Wu, A.W.; Chem. Commum. 1995, 2551; Wang, X. J.; Tan, J.; Grozinger, K.; Tetrahedron Lett. 1998, 39, 6609.

26. McNulty, J.; Mo, R.; Chem. Commun. 1998, 933.

27. Trost, B. M.; Pulley, S. R.; J. Am. Chem. Soc. 1975, 117, 10143.

28. For some examples of Morita-Baylis-Hillman reactions catalyzed by ionic liquids, see: Dupont, J.; J. Braz. Chem. Soc. 2004, 15, 341; Rosa, J. N.; Afonso, C. A. M.; Santos, A. G.; Tetrahedron 2001, 57, 4189; Kumar, A.; Pawar, S. S.; J. Mol. Catal. A: Chem. 2003, 208, 33; Kim, E. J.; Ko, S. Y.; Song, C. E.; Helv. Chim. Acta 2003, 86, 894; Pegot, B.; Vo-Thanh, G.; Gori, D.; Loupy, A.; Tetrahedron Lett. 2004, 45, 6425; Mi, X. L.; Luo, S. Z.; Cheng, J. P.; J. Org. Chem. 2005, 70, 2338.

29. For some insights related to the role of ionic liquids increasing the rate of the Morita-Baylis-Hillman reaction, see: Santos, L. S.; da Silveira Neto, B. A.; Consorti, C. S.; Pavam, C. H.; Almeida, W. P.; Coelho, F.; Dupont, J.; Eberlin, M. N.; J. Phys. Org. Chem. 2006, 19, 731.

30. Iodide 60 was easily prepared, in almost quantitative yield, through the treatment of bromide $\mathbf{5 8}$ with $\mathrm{NaI}$ in acetone.

31. Nicoletti, M.; O'Hagan, D.; Slawin, A. M. Z.; J. Chem. Soc. Perkin Trans. I 2002, 116 and references cited therein; Yoshida, M.; Watanabe, T.; Ishikawa, T.; Heterocycles 2001, 54, 433; Banwell, M. G.; Harvey, J. E.; Hockless, D. C. R; Wu, A. W.; J. Org. Chem. 2000, 65, 4241.

Received: July 24, 2007

Web Release Date: November 15, 2007

FAPESP helped in meeting the publication costs of this article. 